Supporting Information for

\title{
Developing a Gel-Based Sensor Using Crystal Morphology Prediction
}

Gesine K. Veits, Kelsey K. Carter, Sarah J. Cox, and Anne J. McNeil*

Department of Chemistry and Macromolecular Science and Engineering Program

University of Michigan, 930 North University Avenue, Ann Arbor, Michigan 48109-1055, United

States

\section{Contents}

Page

I. Materials

S2

II. General Procedures

S2

III. Synthetic Procedures

IV. ${ }^{1} \mathrm{H}$ and ${ }^{13} \mathrm{C}$ NMR Spectra

S10

V. Cambridge Structural Database Search Method

S24

VI. Morphology Prediction Method

S26

VII. Geometry Optimization

S33

VIII. Morphology Prediction

IX. A Closer Look at the Top 5\% of Attachment Energy-predicted Aspect Ratios

S50

$X$. Impact of Electron Delocalization on Morphology Prediction

S54

XI. Gel Formation Experiments

S55

XII. Rheological Characterization of Gels

S57

XIII. Gel Characterization via Scanning Electron Microscopy

S61

XIV. Powder X-Ray Diffraction Characterization

S65

XV. In-situ Gelation Experiments with Paint

S66

XVI. Gelation with Different Lead(II) Sources

S68

XVII. Testing Gelation with Other Metal Salts

S69

XVIII.Testing Gelation with Lead(II) in the Presence of Other Metal Salts

S70

XIX. Developing a Sensor for 5000 ppm Pb in paint

S71

$\mathrm{XX}$. Developing a sensor for $\mathrm{Pb}$ at $5000 \mathrm{ppm}$ in dry paint

S77

$X X I$. Effect of excess ligand and $\mathrm{Cu}(\mathrm{OAc})_{2}$ on gel formation

S79

$X X I I$. Comparing the gel-based sensor to LeadCheck ${ }^{\mathrm{TM}}$ 


\section{Materials}

All reagent grade materials and solvents were purchased from Sigma Aldrich, Acros Organics, or TCl. The paint thinner used was Klean-Strip paint thinner made with mineral spirits. Paints used were as follows: black oil-based paint: Rust-Oleum Professional, V7579 Gloss Black, High performance enamel; pink latex-based paint: Valspar Satin Berry Twist 530832, Spring 2014; white oil-based paint: Rust-Oleum, 7792 Gloss White, Protective Enamel. All alkyl amines and carbon disulfide were distilled prior to use. Methanol was dried over activated molecular sieves under $\mathrm{N}_{2}$ overnight. All other compounds were used without further purification unless otherwise noted. Compounds S1-S3, ${ }^{1} \mathbf{1} \mathbf{a}-\mathbf{h},,^{2,3} \mathbf{2} \mathbf{a}-\mathbf{b},{ }^{4}$ and $\mathbf{3}^{5}$ were prepared from modified literature procedures. Throughout this document $\mathrm{H}_{2} \mathrm{O}$ refers to deionized $\mathrm{H}_{2} \mathrm{O}$, unless otherwise noted.

\section{General Procedures}

${ }^{1} \mathrm{H}$ and ${ }^{13} \mathrm{C}$ NMR spectra for all compounds were acquired in deuterated solvents on either a Varian VNMRS 700 operating at 700 and $176 \mathrm{MHz}$, a Varian VNMRS 500 operating at 500 and $126 \mathrm{MHz}$, or a Varian MR400 operating at $400 \mathrm{MHz}$. The chemical shift data are reported in units of $\delta(\mathrm{ppm})$ relative to tetramethylsilane and referenced by residual protic solvent. An asterisk was used to indicate residual $\mathrm{H}_{2} \mathrm{O}$ in all spectra while double bars are used to indicate peaks that have been truncated. Multiplicities are reported as follows: singlet (s), doublet (d), triplet $(\mathrm{t})$, quartet $(\mathrm{q})$, septet (sept), doublet of doublets (dd), triplet of quartets (tq), doublet of doublet of triplets (ddt) and multiplet $(\mathrm{m})$.

High-Resolution Mass Spectrometry (HRMS) data were obtained on a Micromass AutoSpec Ultima Magnetic Sector mass spectrometer via electron impact ionization on a desorption probe or via electrospray ionization.

Scanning Electron Microscopy (SEM) images were obtained on a Hitachi S3200N SEM using a $15-k V$ accelerating voltage or a FEI NOVA Nanolab Dualbeam Workstation with a Schottky field emitter operated at 10-20 kV accelerating voltage.

Powder X-Ray Diffraction data were collected at ambient temperature using a Bruker D8 Advance diffractometer with a LynxEye detector using graphite monochromated Cu-Ka radiation $(1.5406 \AA$ ) operating at $40 \mathrm{kV}, 40 \mathrm{~mA}$ and on a Rigaku SmartLab diffractometer in reflection mode using point-focus $\mathrm{Cu}-\mathrm{Ka}$ radiation $(\lambda=1.5425035)$ operating at $40 \mathrm{kV}, 44 \mathrm{~mA}$ and equipped with a Pilatus $100 \mathrm{~K} / \mathrm{R}$ 2-dimensional detector set at a nominal sample to detector distance of $200 \mathrm{~mm}$. The samples were analyzed on glass microscope slides with wells.

Cambridge Structural Database (CSD) - All CIF files were used without alteration unless otherwise noted. The list of 184 crystal structures analyzed in this work can be found in Table S1. Each crystal structure is identified through its six-letter tag (e.g., NAYNUW). 
Materials Studio - All calculations were performed with Materials Studio 6.0 by Accelrys Software Inc. ${ }^{6}$ The aspect ratios (AR) were calculated by dividing the longest distance by the shortest distance within a crystal as depicted in Scheme S1.

\section{Scheme S1.}

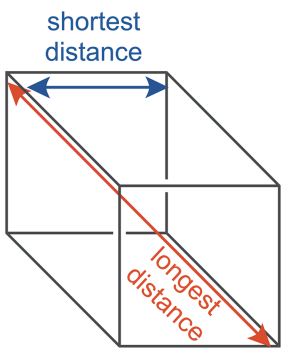

$\mathrm{AR}=\frac{\text { longest distance }}{\text { shortest distance }}$ 


\section{Synthetic Procedures}

A. Synthesis of amines

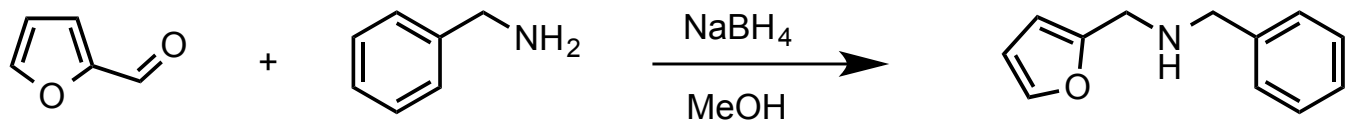

S1

$\mathbf{N}$-Benzyl-1-(furan-2-yl)methanamine (S1). 2-Furaldehyde $(300 \mu \mathrm{L}, \quad 3.63 \mathrm{mmol})$ and benzylamine $(360 \mu \mathrm{L}, 3.30 \mathrm{mmol})$ were combined in dry $\mathrm{MeOH}(9 \mathrm{~mL})$ and stirred under $\mathrm{N}_{2}$ for $18 \mathrm{~h}$. The solution was then treated with $\mathrm{NaBH}_{4}(279 \mathrm{mg}, 7.38 \mathrm{mmol})$ in small portions, and stirred under $\mathrm{N}_{2}$. After $\sim 1 \mathrm{~h}$, no starting material was visible by TLC. The reaction was carefully quenched with $\mathrm{H}_{2} \mathrm{O}(10 \mathrm{~mL})$. $\mathrm{MeOH}$ was removed via rotary evaporation, and the aqueous residue extracted with EtOAc $(3 \times 10 \mathrm{~mL})$. The combined organic layers were dried over $\mathrm{MgSO}_{4}$, filtered, and the solvent removed via rotary evaporation. The resulting oil was purified by flash column chromatography, eluting with $14 \%$ to $20 \%$ EtOAc in hexanes to give a clear oil (518 mg, 84\%). HRMS (ESI): Calcd for $\mathrm{C}_{12} \mathrm{H}_{14} \mathrm{NO}^{+}$, 188.1070. Found, 188.1066.<smiles>COc1ccc(C=O)cc1</smiles><smiles>COc1ccc(CNCc2ccccc2)cc1</smiles>

S2

N-Benzyl-1-(4-methoxyphenyl)methanamine (S2). 4-Methoxybenzaldehyde (740 $\mu \mathrm{L}, 6.08$ $\mathrm{mmol})$ and benzylamine $(600 \mu \mathrm{L}, 5.49 \mathrm{mmol})$ were combined in dry $\mathrm{MeOH}(15 \mathrm{~mL})$ and stirred under $\mathrm{N}_{2}$ for $18 \mathrm{~h}$. The solution was then treated with $\mathrm{NaBH}_{4}(420 . \mathrm{mg}, 11.1 \mathrm{mmol})$ in small portions, and stirred under $\mathrm{N}_{2}$. After $\sim 1 \mathrm{~h}$, no starting material was visible by TLC. The reaction was carefully quenched with $\mathrm{H}_{2} \mathrm{O}(10 \mathrm{~mL})$. MeOH was removed via rotary evaporation, and the aqueous residue extracted with $\mathrm{CH}_{2} \mathrm{Cl}_{2}(3 \times 10 \mathrm{~mL})$. The combined organic layers were dried over $\mathrm{MgSO}_{4}$, filtered, and the solvent removed via rotary evaporation. The resulting oil was purified by flash column chromatography, eluting with $20 \%$ to $33 \%$ EtOAc in hexanes to give a clear oil (1068 mg, 86\%). HRMS (ESI): Calcd for $\mathrm{C}_{15} \mathrm{H}_{18} \mathrm{NO}^{+}, 228.1383$. Found, 228.1380.<smiles>CO[14CH2]C/C=C\CN</smiles><smiles>Cc1cc(C)c(CNCc2ccccc2)c([N+](=O)[O-])c1</smiles>

N-Benzyl-1-mesitylmethanamine (S3). Mesitaldehyde (890 $\mu \mathrm{L}, 6.00 \mathrm{mmol})$ and benzylamine $(600 \mu \mathrm{L}, 5.49 \mathrm{mmol})$ were combined in dry $\mathrm{MeOH}(15 \mathrm{~mL})$ and stirred under $\mathrm{N}_{2}$ for $19 \mathrm{~h}$. The solution was then treated with $\mathrm{NaBH}_{4}(420 . \mathrm{mg}, 11.1 \mathrm{mmol})$ in small portions, and stirred under $\mathrm{N}_{2}$. After $\sim 1 \mathrm{~h}$, no starting material was visible by TLC. The reaction was carefully quenched with $\mathrm{H}_{2} \mathrm{O}(10 \mathrm{~mL})$. MeOH was removed via rotary evaporation, and the aqueous residue extracted with EtOAc $(3 \times 15 \mathrm{~mL})$. The combined organic layers were dried over $\mathrm{MgSO}_{4}$, filtered, and the solvent removed via rotary evaporation. The resulting oil was purified by flash column 
chromatography, eluting with 14\% EtOAc in hexanes to give a light yellow oil (1059 $\mathrm{mg}, 81 \%$ ). HRMS (ESI): Calcd for $\mathrm{C}_{17} \mathrm{H}_{22} \mathrm{~N}^{+}, 240.1747$. Found, 240.1742.

B. Synthesis of lead complexes

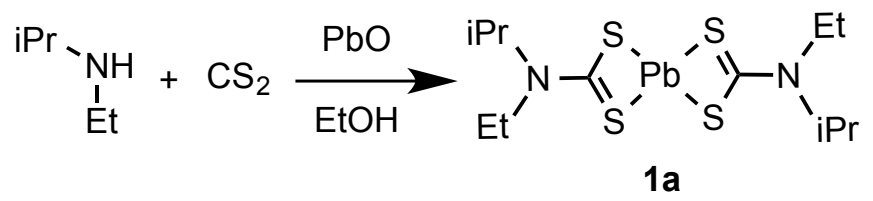

$N^{2}, N^{6}$-Diethyl- $N^{2}, N^{6}$-diisopropyl-1 $\lambda^{3}, 3,5 \lambda^{3}, 7$-tetrathia-4-plumbaspiro[3.3] hepta-1,5-diene-

2,6-diamine (1a). $N$-lsopropyl- $N$-ethyl-amine $(150 \mu \mathrm{L}, 1.24 \mathrm{mmol})$ and carbon disulfide $(80 \mu \mathrm{L}$, $1.3 \mathrm{mmol}$ ) were added to a round-bottom flask equipped with a stirbar and dissolved in $\mathrm{EtOH}$ $(14 \mathrm{~mL})$. Lead(II) oxide (138 $\mathrm{mg}, 0.618 \mathrm{mmol}$ ) was added. The flask was sealed and stirred under $\mathrm{N}_{2}$ for $16 \mathrm{~h}$, forming a white precipitate. Then, $\mathrm{CH}_{2} \mathrm{Cl}_{2}(15 \mathrm{~mL})$ was added to dissolve the precipitate and was subsequently filtered through Celite, washing with $\mathrm{CH}_{2} \mathrm{Cl}_{2}(\sim 50 \mathrm{~mL})$ to remove unreacted $\mathrm{PbO}$. The filtrate was concentrated via rotary evaporation, yielding an offwhite solid (310 mg, 94\%). HRMS (El): Calcd for $\mathrm{C}_{12} \mathrm{H}_{24} \mathrm{~N}_{2} \mathrm{PbS}_{4}{ }^{+}, 532.0583$. Found, 532.0570.

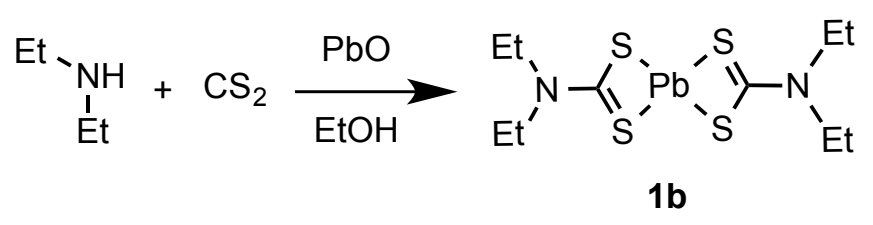

$N^{2}, N^{2}, N^{6}, N^{6}$-Tetraethyl-1 $\lambda^{3}, 3,5 \lambda^{3}, 7$-tetrathia-4-plumbaspiro[3.3]hepta-1,5-diene-2,6-diamine (1b). $N, N$-Diethylamine $(130 \mu \mathrm{L}, 1.26 \mathrm{mmol})$ and carbon disulfide $(80 \mu \mathrm{L}, 1.3 \mathrm{mmol})$ were added to a round-bottom flask equipped with a stirbar and dissolved in EtOH (14 mL). Lead(II) oxide (140. $\mathrm{mg}, 0.627 \mathrm{mmol}$ ) was added. The flask was sealed and stirred under $\mathrm{N}_{2}$ for $16 \mathrm{~h}$, forming a precipitate. Then, $\mathrm{CH}_{2} \mathrm{Cl}_{2}(20 \mathrm{~mL})$ was added to dissolve the precipitate and was subsequently filtered through Celite washing with $\mathrm{CH}_{2} \mathrm{Cl}_{2}(\sim 75 \mathrm{~mL})$ to remove unreacted $\mathrm{PbO}$. The filtrate was concentrated via rotary evaporation, yielding a gray solid $(272 \mathrm{mg}, 86 \%)$. HRMS (EI): Calcd for $\mathrm{C}_{10} \mathrm{H}_{20} \mathrm{~N}_{2} \mathrm{PbS}_{4}{ }^{+}$, 504.0270. Found, 504.0286.

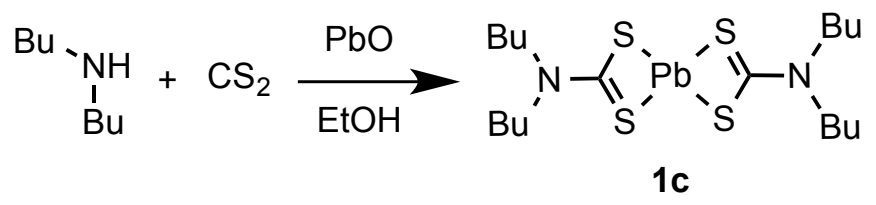

$N^{2}, N^{2}, N^{6}, N^{6}$-Tetrabutyl-1 $\lambda^{3}, 3,5 \lambda^{3}, 7$-tetrathia-4-plumbaspiro[3.3]hepta-1,5-diene-2,6-diamine (1c). $N, N$-Dibutylamine $(210 \mu \mathrm{L}, 1.25 \mathrm{mmol})$ and carbon disulfide $(80 \mu \mathrm{L}, 1.3 \mathrm{mmol})$ were added to a round-bottom flask equipped with a stirbar and dissolved in EtOH (14 mL). Lead(II) oxide (140. $\mathrm{mg}, 0.627 \mathrm{mmol}$ ) was added. The flask was sealed and stirred under $\mathrm{N}_{2}$ for $16 \mathrm{~h}$, forming a white precipitate. Then, $\mathrm{CH}_{2} \mathrm{Cl}_{2}(20 \mathrm{~mL})$ was added to dissolve the precipitate and was subsequently filtered through Celite washing with $\mathrm{CH}_{2} \mathrm{Cl}_{2}(\sim 50 \mathrm{~mL})$ to remove unreacted $\mathrm{PbO}$. 
The filtrate was concentrated via rotary evaporation, yielding a yellow solid (372 $\mathrm{mg}, 97 \%$ ). HRMS (El): Calcd for $\mathrm{C}_{18} \mathrm{H}_{36} \mathrm{~N}_{2} \mathrm{PbS}_{4}{ }^{+}$616.1522. Found, 616.1545.

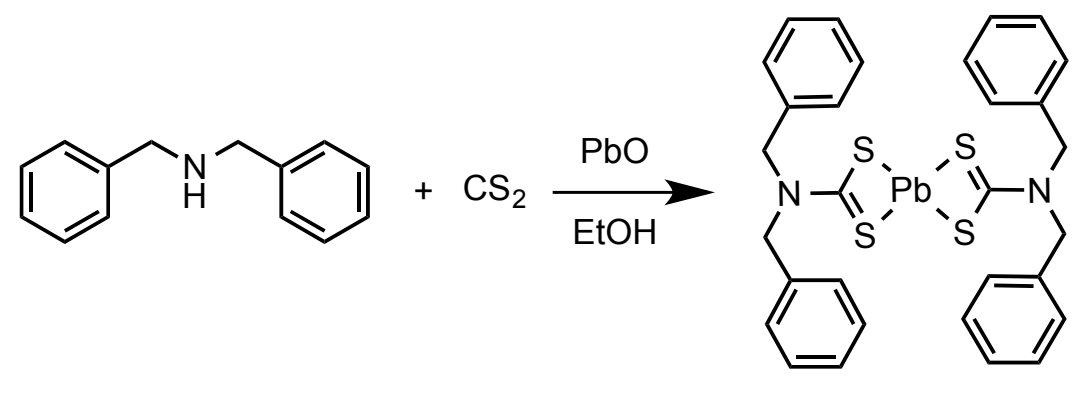

1d

$N^{2}, N^{2}, N^{6}, N^{6}$-Tetrabenzyl-1 $\lambda^{3}, 3,5 \lambda^{3}, 7$-tetrathia-4-plumbaspiro[3.3]hepta-1,5-diene-2,6-

diamine (1d). $N, N$-Dibenzylamine $(100 \mu \mathrm{L}, 0.520 \mathrm{mmol})$ and carbon disulfide $(50 \mu \mathrm{L}, 0.83$ $\mathrm{mmol}$ ) were added to a round-bottom flask equipped with a stirbar and dissolved in EtOH (6 $\mathrm{mL})$. Lead(II) oxide $(58.3 \mathrm{mg}, 0.261 \mathrm{mmol})$ was added. The flask was sealed and stirred under $\mathrm{N}_{2}$ for $16 \mathrm{~h}$, forming a white precipitate. Then, $\mathrm{CH}_{2} \mathrm{Cl}_{2}(50 \mathrm{~mL})$ was added to dissolve the precipitate and was subsequently filtered washing with $\mathrm{CH}_{2} \mathrm{Cl}_{2}(\sim 150 \mathrm{~mL})$ to remove unreacted $\mathrm{PbO}$. The filtrate was concentrated via rotary evaporation, yielding a white solid $(182.0 \mathrm{mg}$, 93\%). HRMS (El): Calcd for $\mathrm{C}_{30} \mathrm{H}_{28} \mathrm{~N}_{2} \mathrm{PbS}_{4}{ }^{+}, 752.0896$. Found, 752.0898.<smiles>C=CCNCC=C</smiles>
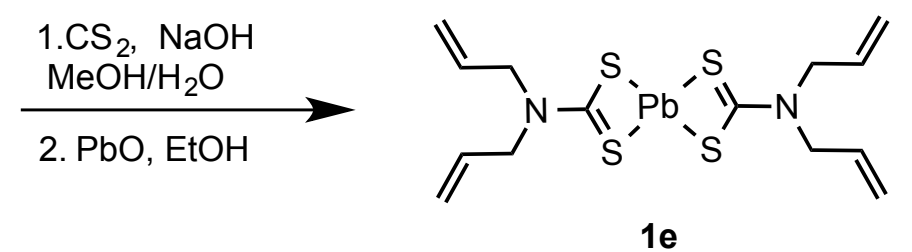

$N^{2}, N^{2}, N^{6}, N^{6}$-Tetraallyl-1 $\lambda^{3}, 3,5 \lambda^{3}, 7$-tetrathia-4-plumbaspiro[3.3] hepta-1,5-diene-2,6-diamine (1e). A solution of $\mathrm{NaOH}(65 \mathrm{mg}, 1.6 \mathrm{mmol})$ in $\mathrm{H}_{2} \mathrm{O}(0.1 \mathrm{~mL})$ and $\mathrm{MeOH}(6 \mathrm{~mL})$ was stirred at 0 ${ }^{\circ} \mathrm{C}$ for $5 \mathrm{~min}$ prior to adding diallylamine $(200 \mu \mathrm{L}, 1.62 \mathrm{mmol})$. After $10 \mathrm{~min}$, carbon disulfide (100 $\mu \mathrm{L}, 1.62 \mathrm{mmol}$ ) was added. The reaction was allowed to come to rt and stirred for $18 \mathrm{~h}$. The solvent was removed to give a yellow oil.

A portion of the crude product $(50.0 \mathrm{mg}, 0.256 \mathrm{mmol})$ was dissolved in $\mathrm{EtOH}(3 \mathrm{~mL})$ and treated with $\mathrm{PbO}(28.6 \mathrm{mg}, 0.128 \mathrm{mmol})$. The flask was sealed and stirred under $\mathrm{N}_{2}$ for $16 \mathrm{~h}$, forming a white precipitate. Then, $\mathrm{CH}_{2} \mathrm{Cl}_{2}(5 \mathrm{~mL})$ was added to dissolve the precipitate and was subsequently filtered through Celite, washing with $\mathrm{CH}_{2} \mathrm{Cl}_{2}(\sim 50 \mathrm{~mL})$ to remove unreacted $\mathrm{PbO}$. The filtrate was concentrated via rotary evaporation, yielding a white solid $(67.8 \mathrm{mg}, 96 \%)$. *Note that the product is not stable in $\mathrm{CH}_{2} \mathrm{Cl}_{2}$ so exposure should be minimized. HRMS (EI): $\mathrm{C}_{14} \mathrm{H}_{20} \mathrm{~N}_{2} \mathrm{PbS}_{4}{ }^{+}, 552.0270$. Found, 552.0276. 


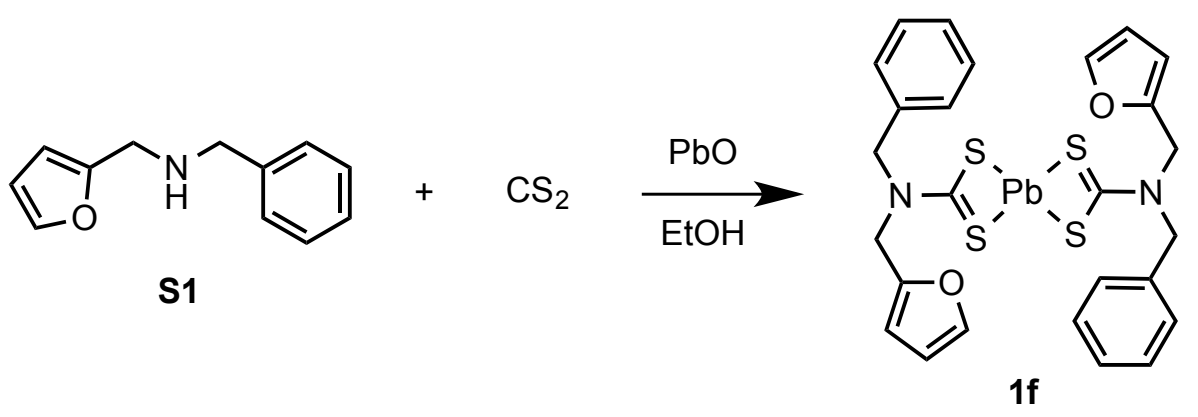

$N^{2}, N^{6}$-Dibenzyl- $N^{2}, N^{6}$-bis(furan-2-ylmethyl)-1 $\lambda^{3}, 3,5 \lambda^{3}, 7$-tetrathia-4-plumbaspiro[3.3] hepta1,5-diene-2,6-diamine (1f). Amine S1 $(99.7 \mathrm{mg}, 0.530 \mathrm{mmol})$ and carbon disulfide $(60 \mu \mathrm{L}, 1.1$ $\mathrm{mmol}$ ) were added to a round-bottom flask equipped with a stirbar and dissolved in EtOH (6 $\mathrm{mL})$. Lead(II) oxide $(59.3 \mathrm{mg}, 0.266 \mathrm{mmol})$ was added. The flask was sealed and stirred under $\mathrm{N}_{2}$ for $21 \mathrm{~h}$, forming a white precipitate. Then, $\mathrm{CH}_{2} \mathrm{Cl}_{2}(\sim 15 \mathrm{~mL})$ was added to dissolve the precipitate and was subsequently filtered through Celite, washing with $\mathrm{CH}_{2} \mathrm{Cl}_{2}(\sim 50 \mathrm{~mL})$ to remove unreacted $\mathrm{PbO}$. The filtrate was was concentrated via rotary evaporation, yielding a white solid (187 mg, 97\%). HRMS (El): $\mathrm{C}_{26} \mathrm{H}_{24} \mathrm{~N}_{2} \mathrm{O}_{2} \mathrm{PbS}_{4}{ }^{+}, 732.0481$. Found, 732.0468 .

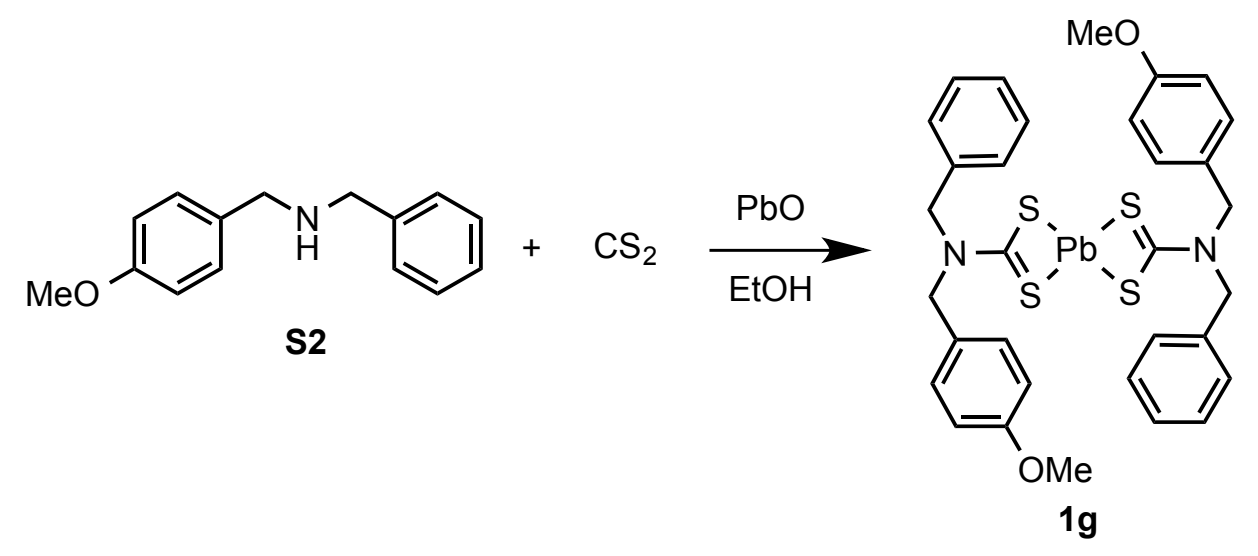

$N^{2}, N^{6}$-Dibenzyl- $N^{2}, N^{6}$-bis (4-methoxybenzyl)-1 $\lambda^{3}, 3,5 \lambda^{3}, 7$-tetrathia-4-plumbaspiro[3.3] hepta1,5-diene-2,6-diamine (19). Amine S2 (150. $\mathrm{mg}, 0.660 \mathrm{mmol}$ ) and carbon disulfide (60 $\mu \mathrm{L}, 0.99$ $\mathrm{mmol}$ ) were added to a round-bottom flask equipped with a stirbar and dissolved in $\mathrm{EtOH}(6$ $\mathrm{mL})$. Lead(II) oxide $(73.9 \mathrm{mg}, 0.330 \mathrm{mmol})$ was added. The flask was sealed and stirred under $\mathrm{N}_{2}$ for $18 \mathrm{~h}$, forming a white precipitate. Then, $\mathrm{CH}_{2} \mathrm{Cl}_{2}(\sim 20 \mathrm{~mL})$ was added to dissolve the precipitate and was subsequently filtered through Celite, washing with $\mathrm{CH}_{2} \mathrm{Cl}_{2}(\sim 100 \mathrm{~mL})$ to remove unreacted $\mathrm{PbO}$. The filtrate was concentrated via rotary evaporation, yielding a white solid (257 mg, 96\%). HRMS (El): $\mathrm{C}_{32} \mathrm{H}_{32} \mathrm{~N}_{2} \mathrm{O}_{2} \mathrm{PbS}_{4}{ }^{+}, 812.1107$. Found, 812.1133. 


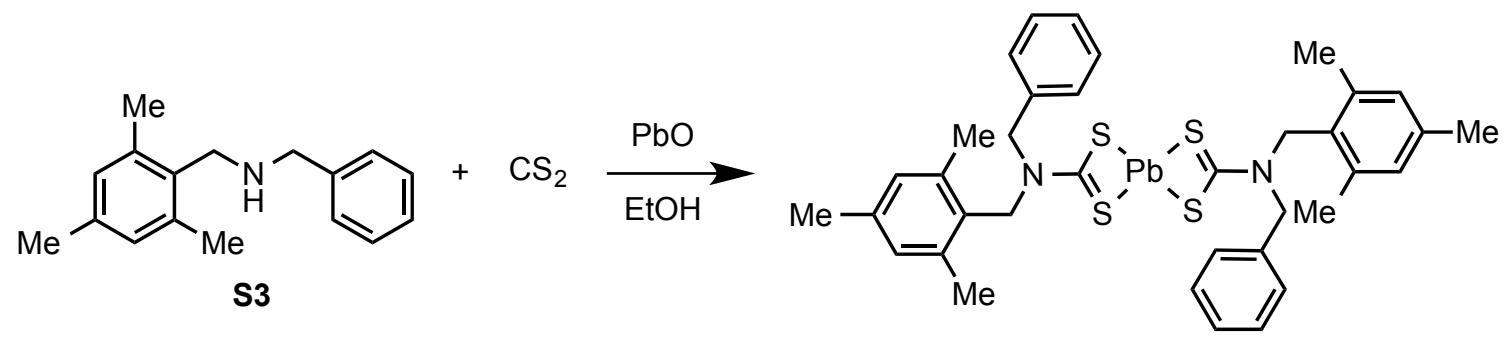

$1 \mathrm{~h}$

$N^{2}, N^{6}$-Dibenzyl- $N^{2}, N^{6}$-bis(2,4,6-trimethylbenzyl)- $1 \lambda^{3}, 3,5 \lambda^{3}, 7$-tetrathia-4-

plumbaspiro[3.3]hepta-1,5-diene-2,6-diamine (1h). Amine $\mathbf{S 3}$ (150. $\mathrm{mg}, 0.630 \mathrm{mmol}$ ) and carbon disulfide $(60 \mu \mathrm{L}, 1.0 \mathrm{mmol})$ were added to a round-bottom flask equipped with a stirbar and dissolved in EtOH (6 mL). Lead(II) oxide $(70.0 \mathrm{mg}, 0.310 \mathrm{mmol})$ was added. The flask was sealed and stirred under $\mathrm{N}_{2}$ for $21 \mathrm{~h}$, forming a white precipitate. Then, $\mathrm{CH}_{2} \mathrm{Cl}_{2}(\sim 25 \mathrm{~mL})$ was added to dissolve the precipitate and was subsequently filtered through Celite, washing with $\mathrm{CH}_{2} \mathrm{Cl}_{2} \quad(\sim 100 \mathrm{~mL})$ to remove unreacted $\mathrm{PbO}$. The filtrate was concentrated via rotary evaporation, yielding a white solid that was a 1:1 mixture of starting material to desired product by ${ }^{1} \mathrm{H}$ NMR spectroscopy. After purifying by flash column chromatography, the desired product was isolated as a white solid (113 mg, 44\%). HRMS (ESI): $\mathrm{C}_{36} \mathrm{H}_{41} \mathrm{~N}_{2} \mathrm{PbS}_{4}{ }^{+}, 837.1913$. Found, 837.1904.

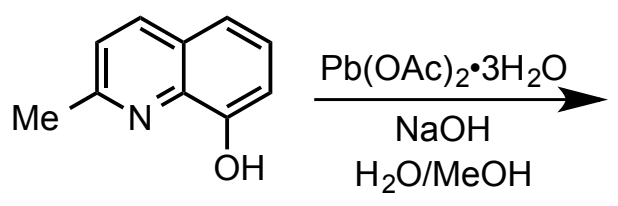<smiles></smiles>

2a

4,4'-dimethyl-3 $3 \lambda^{4}, 3^{\prime} \lambda^{4}-2,2$ '-spirobi[[1,3,2]oxazaplumbolo[5,4,3-ij]quinoline] (2a). A roundbottom flask equipped with a stir bar was charged with $\mathrm{H}_{2} \mathrm{O}(9 \mathrm{~mL}), \mathrm{MeOH}(9 \mathrm{~mL})$, and $\mathrm{NaOH}$ $(52.1 \mathrm{mg}, 1.30 \mathrm{mmol})$. 8-Hydroxyquinaldine $(100 \mathrm{mg}, 0.628 \mathrm{mmol})$ was added and stirred to dissolve. Lead(II) acetate trihydrate $(119 \mathrm{mg}, 0.314 \mathrm{mmol})$ was added and stirred for $25 \mathrm{~min}$. A yellow precipitate formed and the solution was filtered, and then washed with $\mathrm{H}_{2} \mathrm{O}(\sim 20 \mathrm{~mL})$ to yield a yellow solid (154.3 mg, 95\%). HRMS (El): Calcd for $\mathrm{C}_{20} \mathrm{H}_{16} \mathrm{~N}_{2} \mathrm{O}_{2} \mathrm{~Pb}^{+}, 524.0973$. Found, 524.0972. 


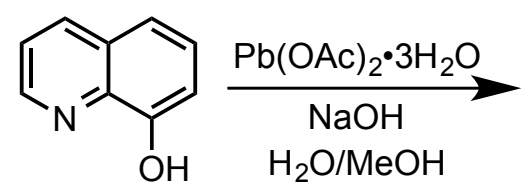<smiles></smiles>

2b

$3 \lambda^{4}, 3^{\prime} \lambda^{4}-2,2^{\prime}$-spirobi[[1,3,2] oxazaplumbolo[5,4,3-ij]quinoline] (2b). A round-bottom flask equipped with a stir bar was charged with $\mathrm{H}_{2} \mathrm{O}(9 \mathrm{~mL}), \mathrm{MeOH}(9 \mathrm{~mL})$, and $\mathrm{NaOH}(54.5 \mathrm{mg}, 1.36$ $\mathrm{mmol})$. 8-Hydroxyquinoline $(90.8 \mathrm{mg}, 0.626 \mathrm{mmol})$ was added and stirred to dissolve. Lead(II) acetate trihydrate $(119 \mathrm{mg}, 0.314 \mathrm{mmol})$ was added and stirred for $20 \mathrm{~min}$. A yellow precipitate formed and the solution was filtered, and then washed with $\mathrm{H}_{2} \mathrm{O}(\sim 20 \mathrm{~mL})$, to yield a yellow solid (151.7 mg, 98\%). HRMS (EI): Calcd for $\mathrm{C}_{18} \mathrm{H}_{12} \mathrm{~N}_{2} \mathrm{O}_{2} \mathrm{~Pb}^{+}, 496.0660$. Found, 496.0661.

C. Synthesis of dithiocarbamate salt

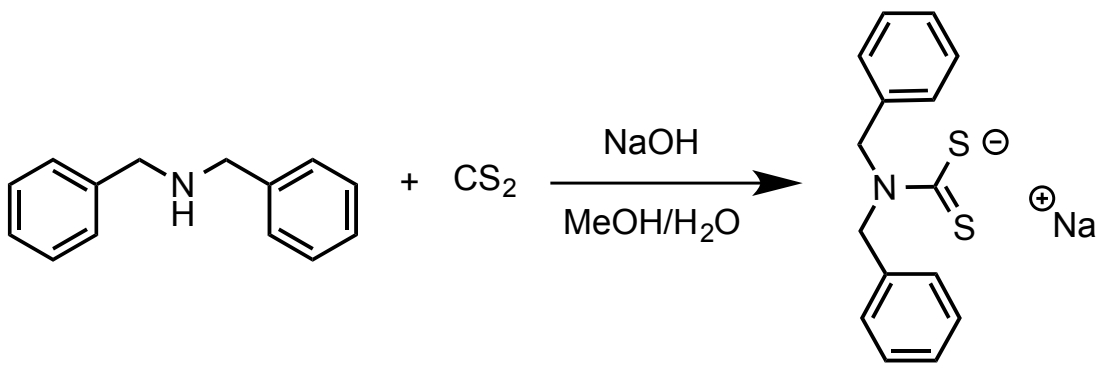

3

Sodium dibenzylcarbamodithioate (3). Sodium hydroxide $(63.2 \mathrm{mg}, 1.58 \mathrm{mmol})$ was dissolved in $\mathrm{H}_{2} \mathrm{O}(0.3 \mathrm{~mL})$ and $\mathrm{MeOH}(6 \mathrm{~mL})$ in a round-bottom flask equipped with a stirbar. The solution was cooled to $0{ }^{\circ} \mathrm{C}$ and dibenzylamine $(300 \mu \mathrm{L}, 1.56 \mathrm{mmol})$ was added. The clear solution stirred vigorously for $\sim 20 \mathrm{~min}$ at $0{ }^{\circ} \mathrm{C}$ before carbon disulfide $(100 \mu \mathrm{L}, 1.66 \mathrm{mmol})$ was added. The yellow solution was allowed to come to rt. The solvent was removed via rotary evaporation, yielding a white/yellow solid containing some water/methanol $(485 \mathrm{mg}, 105 \%$ with residual water). $\mathrm{HRMS}$ (EI): $\mathrm{C}_{15} \mathrm{H}_{14} \mathrm{NS}_{2}{ }^{-}, 272.0573$. Found, 272.0574. 


\section{IV. ${ }^{1} \mathrm{H}$ and ${ }^{13} \mathrm{C}$ NMR Spectra}
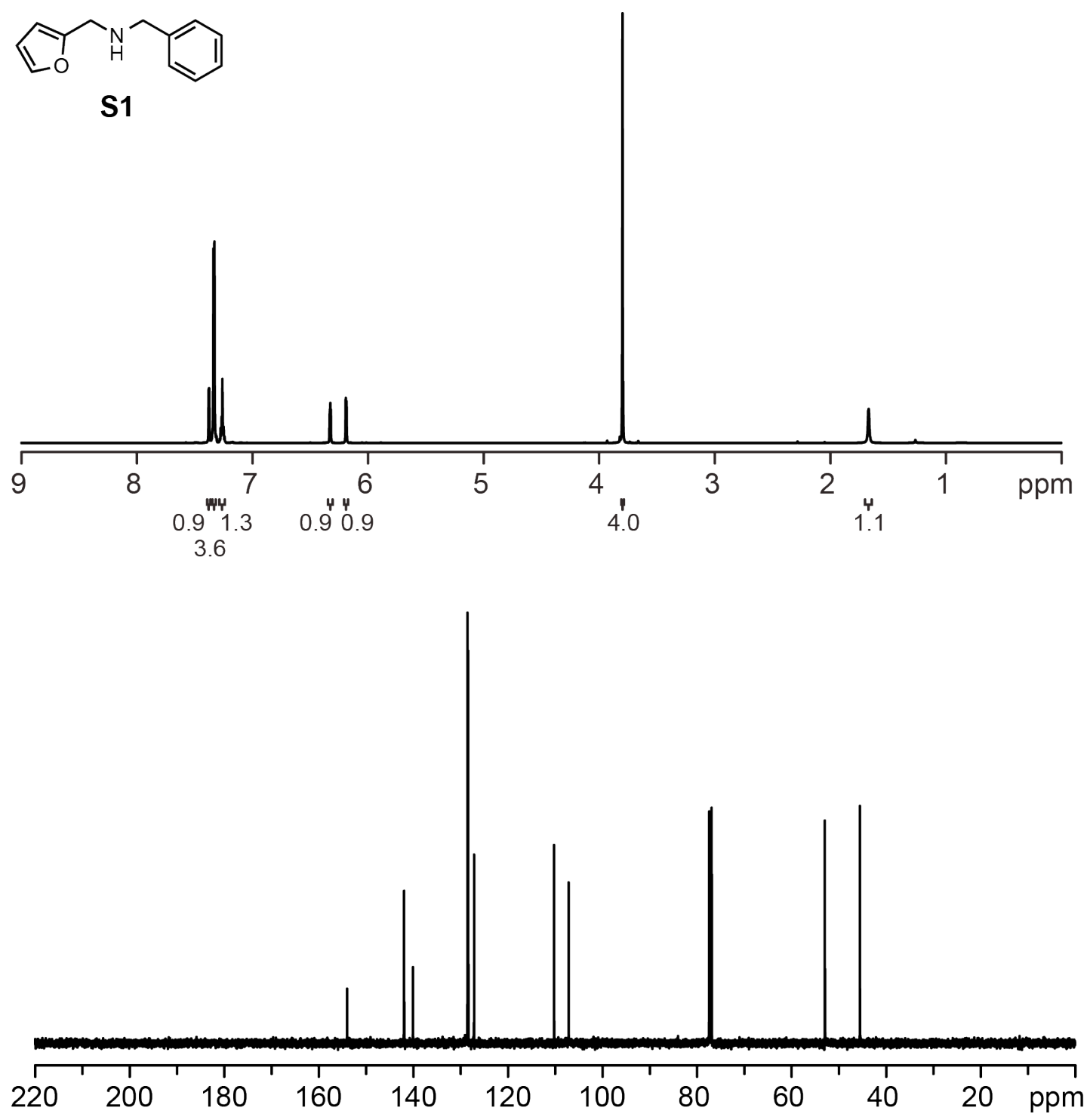

Figure S1. ${ }^{1} \mathrm{H}$ and ${ }^{13} \mathrm{C}$ NMR spectra of S1. ${ }^{1} \mathrm{H}$ NMR $\left(500 \mathrm{MHz}, \mathrm{CDCl}_{3}\right) \delta 7.39-7.37(\mathrm{~m}, 1 \mathrm{H})$, $7.33(\mathrm{~d}, J=4.3 \mathrm{~Hz}, 4 \mathrm{H}), 7.29-7.24(\mathrm{~m}, 1 \mathrm{H}), 6.33(\mathrm{dd}, J=3.0,2.0 \mathrm{~Hz}, 1 \mathrm{H}), 6.19(\mathrm{~d}, J=3.1 \mathrm{~Hz}$, $1 \mathrm{H}), 3.80(\mathrm{~s}, 4 \mathrm{H}), 1.67(\mathrm{~s}, 1 \mathrm{H}) .{ }^{13} \mathrm{C}$ NMR $\left(126 \mathrm{MHz} \mathrm{CDCl}_{3}\right): \delta 154.01,141.95,140.08,128.55$, 128.38, 127.15, 110.23, 107.14, 52.97, 45.55. 
<smiles>COc1ccc(CNCc2ccccc2)cc1</smiles>
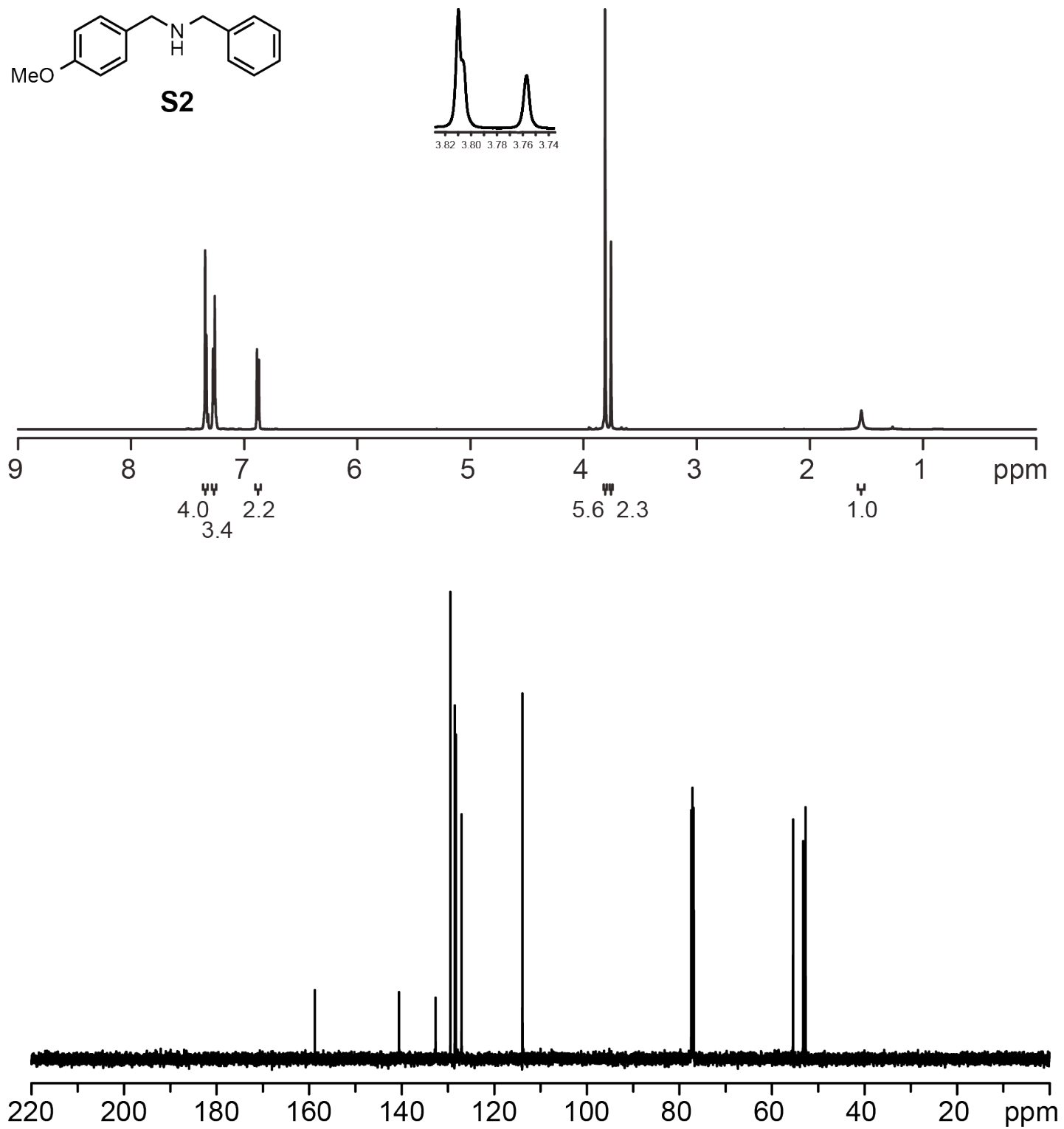

Figure S2. ${ }^{1} \mathrm{H}$ and ${ }^{13} \mathrm{C}$ NMR spectra of S2. ${ }^{1} \mathrm{H}$ NMR $\left(500 \mathrm{MHz}, \mathrm{CDCl}_{3}\right) \delta 7.36-7.31(\mathrm{~m}, 4 \mathrm{H})$, $7.29-7.24(\mathrm{~m}, 3 \mathrm{H}), 6.90-6.86(\mathrm{~m}, 2 \mathrm{H}), 3.81(\mathrm{~s}, 3 \mathrm{H}), 3.81(\mathrm{~s}, 2 \mathrm{H}), 3.76(\mathrm{~s}, 2 \mathrm{H}), 1.54(\mathrm{~s}, 1 \mathrm{H})$. ${ }^{13} \mathrm{C} \mathrm{NMR}\left(126 \mathrm{MHz}, \mathrm{CDCl}_{3}\right): \delta 158.76,140.56,132.65,129.45,128.51,128.28,127.03,113.91$, $55.42,53.23,52.72$. 
<smiles>Cc1cc(C)c(CNCc2ccccc2)c([N+](=O)[O-])c1</smiles>

S3
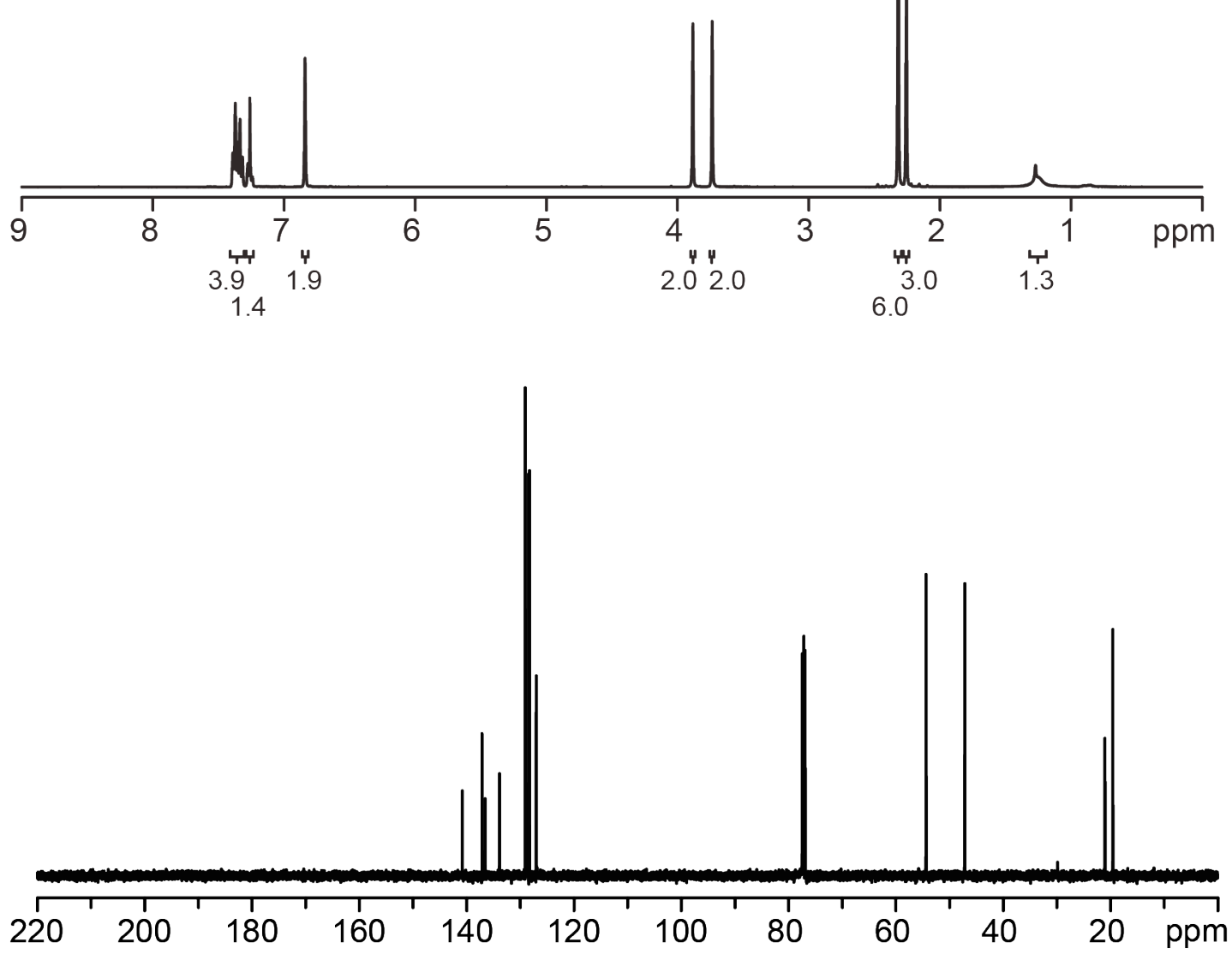

Figure S3. ${ }^{1} \mathrm{H}$ and ${ }^{13} \mathrm{C}$ NMR spectra of S3. ${ }^{1} \mathrm{H}$ NMR $\left(400 \mathrm{MHz}, \mathrm{CDCl}_{3}\right) \delta 7.41-7.30(\mathrm{~m}, 4 \mathrm{H})$, $7.29-7.23(\mathrm{~m}, 1 \mathrm{H}), 6.84(\mathrm{~s}, 2 \mathrm{H}), 3.88(\mathrm{~s}, 2 \mathrm{H}), 3.74(\mathrm{~s}, 2 \mathrm{H}), 2.32(\mathrm{~s}, 6 \mathrm{H}), 2.25(\mathrm{~s}, 3 \mathrm{H}), 1.26(\mathrm{~s}$, 1H). ${ }^{13} \mathrm{C}$ NMR $\left(126 \mathrm{MHz}, \mathrm{CDCl}_{3}\right): \delta 140.80,137.12,136.53,133.86,129.05,128.43,128.26$, 127.01, 54.36, 47.16, 21.03, 19.57. 


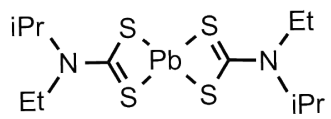

$1 \mathrm{a}$
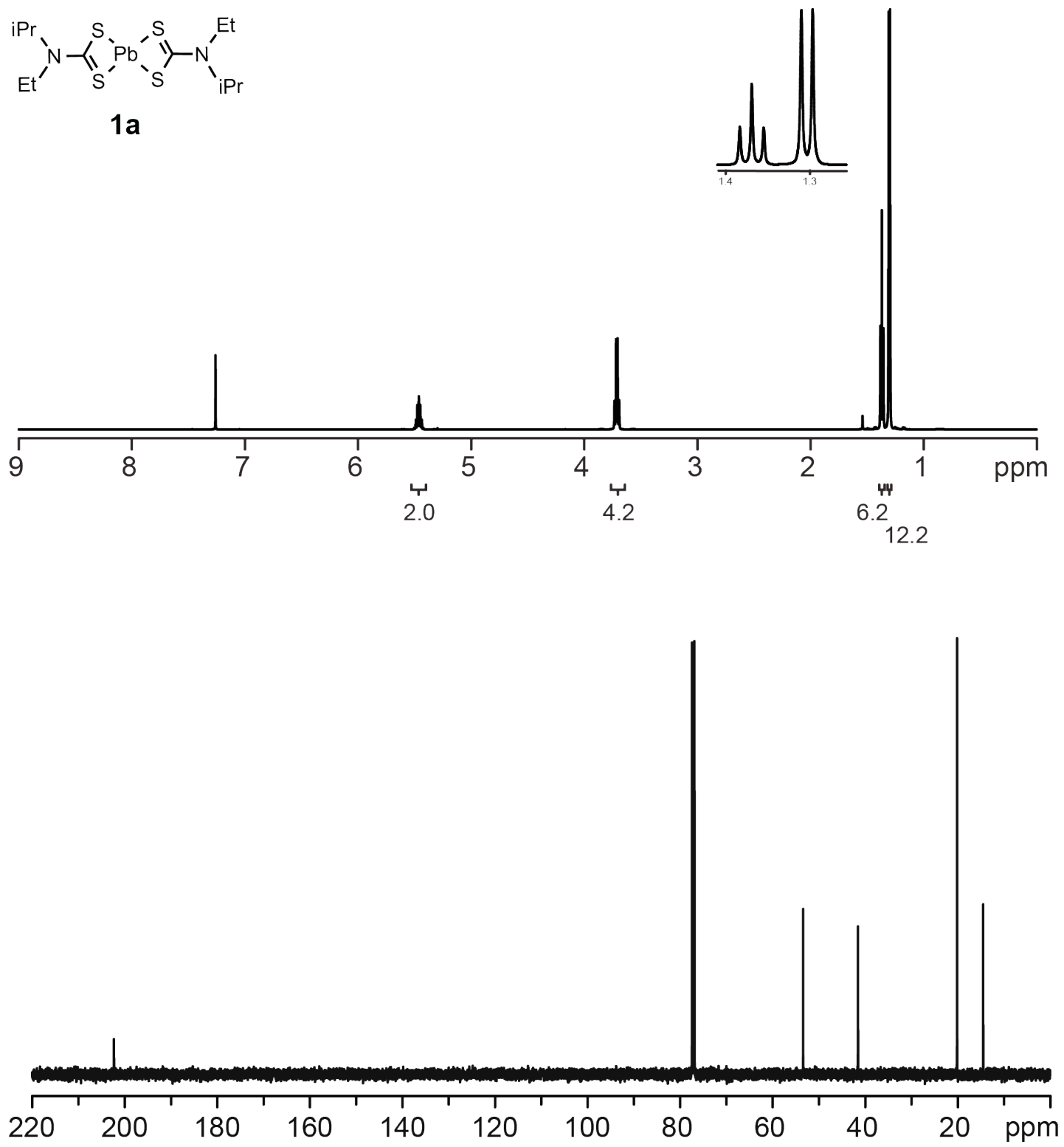

Figure S4. ${ }^{1} \mathrm{H}$ and ${ }^{13} \mathrm{C}$ NMR spectra of 1a. ${ }^{1} \mathrm{H}$ NMR $\left(500 \mathrm{MHz}, \mathrm{CDCl}_{3}\right) \delta 5.46$ (sept, $\mathrm{J}=7 \mathrm{~Hz}$, $2 \mathrm{H}$ ), $3.71(\mathrm{q}, J=7 \mathrm{~Hz}, 4 \mathrm{H}), 1.37(\mathrm{t}, J=7 \mathrm{~Hz}, 6 \mathrm{H}), 1.30(\mathrm{~d}, J=7 \mathrm{~Hz}, 12 \mathrm{H}) .{ }^{13} \mathrm{C}$ NMR $(126 \mathrm{MHz}$, $\left.\mathrm{CDCl}_{3}\right): \delta 202.32,53.41,41.56,20.13,14.50$. 
<smiles>CCN(CC)C1=[SH][PH]2(S1)SC(N(CC)CC)S2</smiles>

1b
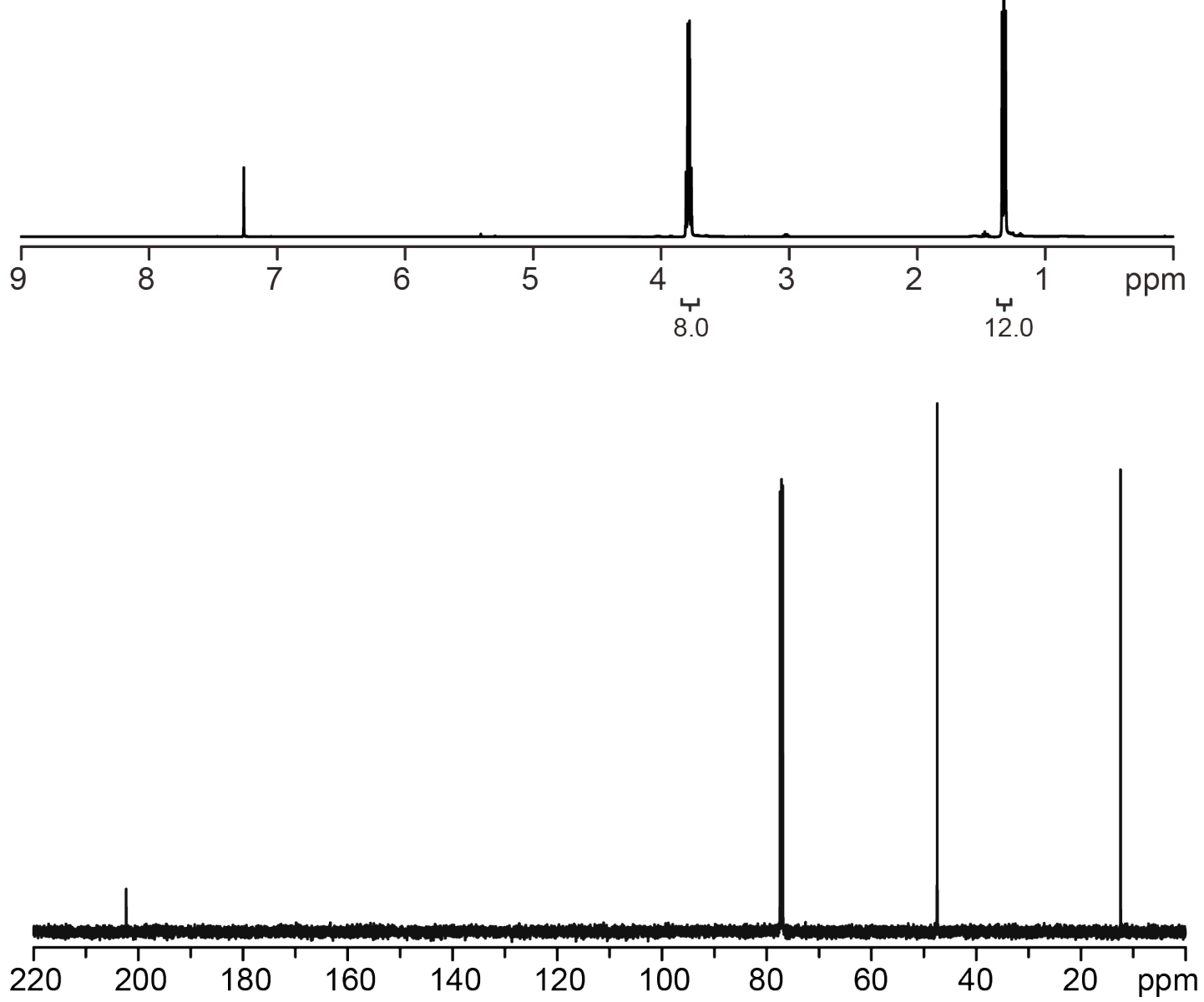

Figure S5. ${ }^{1} \mathrm{H}$ and ${ }^{13} \mathrm{C}$ NMR spectra of 1 b. ${ }^{1} \mathrm{H}$ NMR $\left(500 \mathrm{MHz}, \mathrm{CDCl}_{3}\right) \delta 3.79(\mathrm{q}, \mathrm{J}=7 \mathrm{~Hz}, 8 \mathrm{H})$, $1.32(\mathrm{t}, \mathrm{J}=7 \mathrm{~Hz}, 12 \mathrm{H}) .{ }^{13} \mathrm{C}$ NMR $\left(126 \mathrm{MHz} \mathrm{CDCl}_{3}\right)$ : $\delta 202.27,47.42,12.41$. 

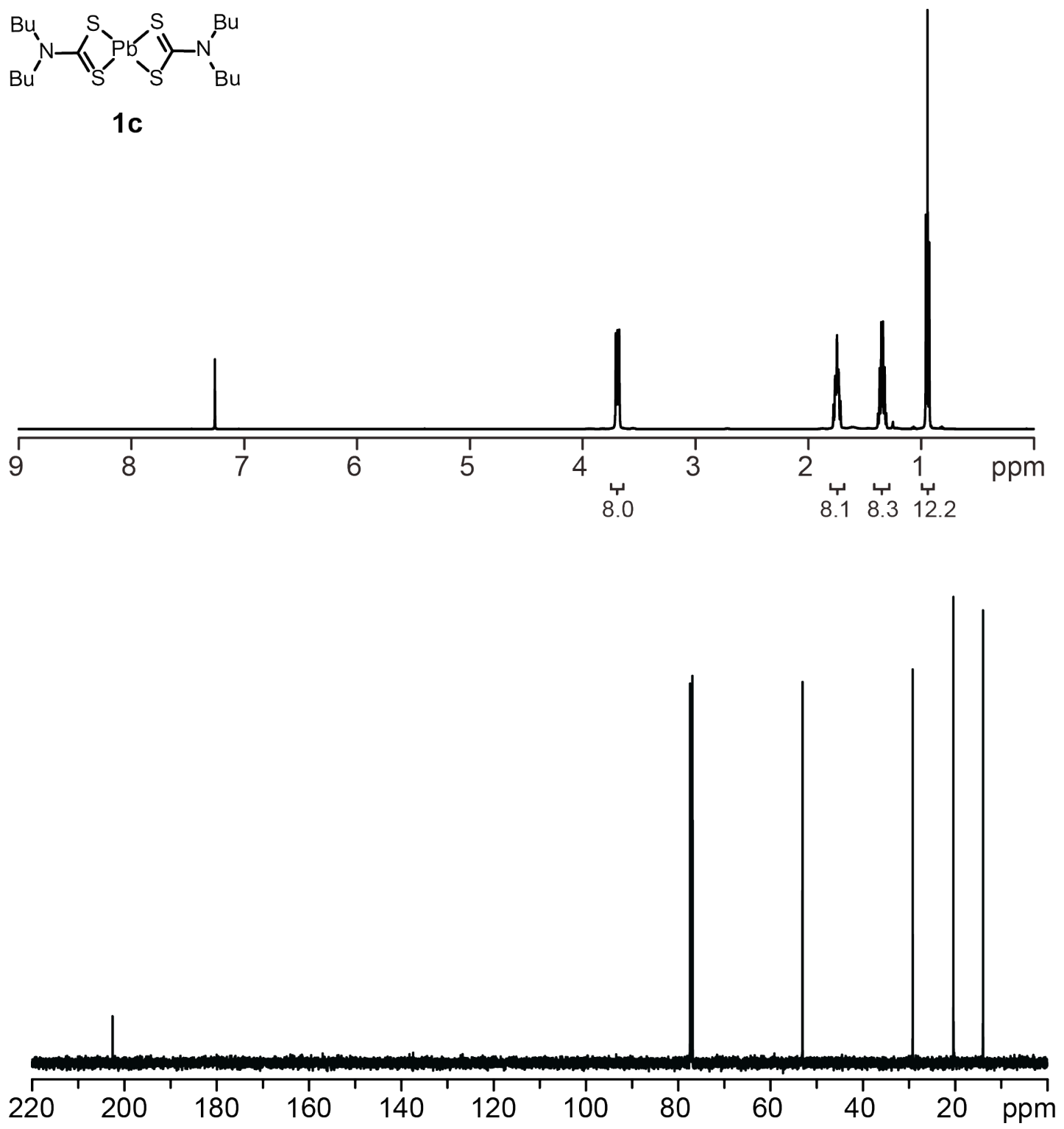

Figure S6. ${ }^{1} \mathrm{H}$ and ${ }^{13} \mathrm{C}$ NMR spectra of 1c. ${ }^{1} \mathrm{H}$ NMR $\left(500 \mathrm{MHz}, \mathrm{CDCl}_{3}\right) \delta 3.72-3.66(\mathrm{~m}, 8 \mathrm{H})$, $1.78-1.71(\mathrm{~m}, 8 \mathrm{H}), 1.35(\mathrm{tq}, J=7,7 \mathrm{~Hz}, 8 \mathrm{H}), 0.94(\mathrm{t}, J=7 \mathrm{~Hz}, 12 \mathrm{H}) .{ }^{13} \mathrm{C}$ NMR $(126 \mathrm{MHz}$, $\left.\mathrm{CDCl}_{3}\right): \delta 202.55,53.07,29.19,20.38,13.95$. 

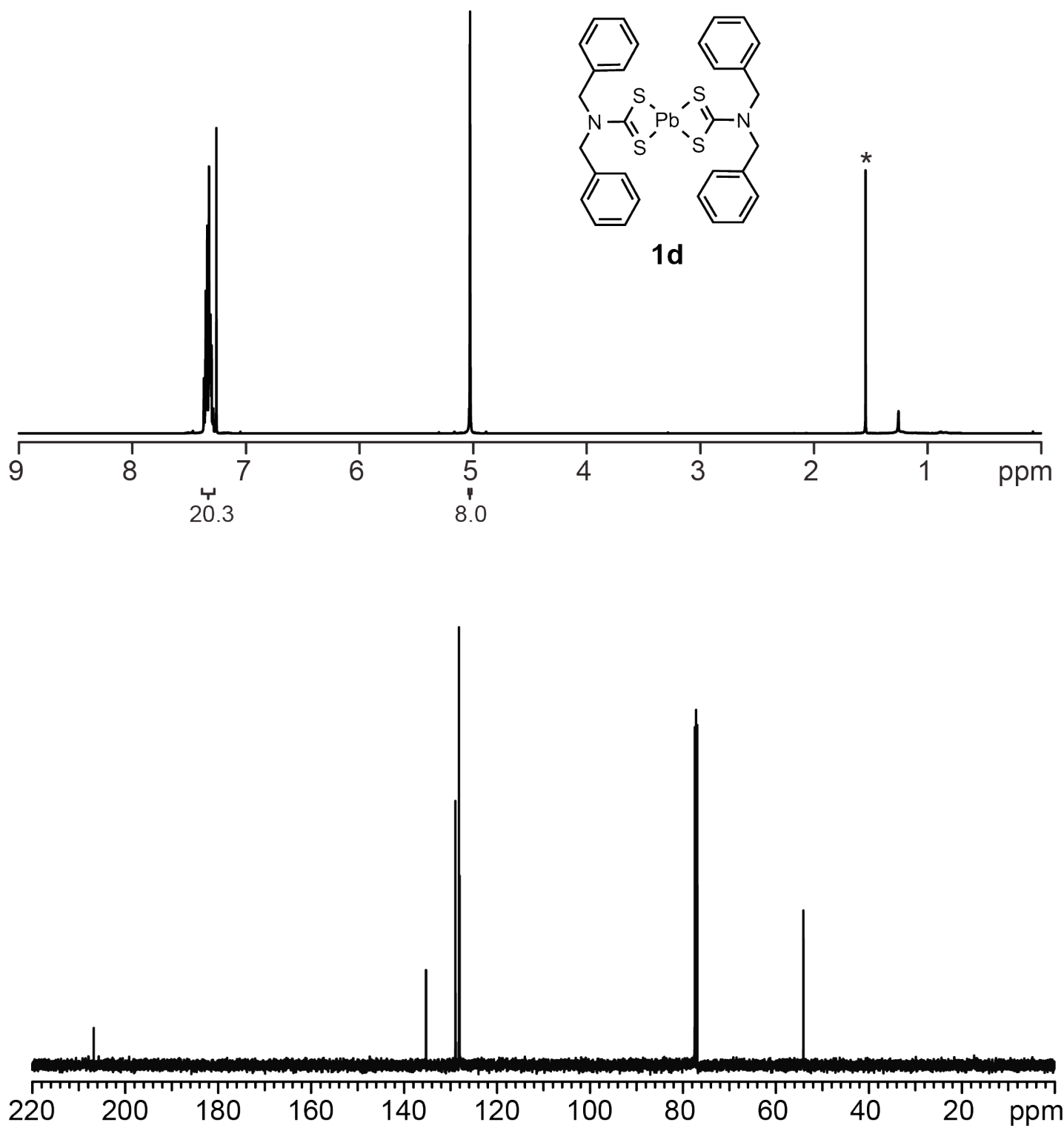

Figure S7. ${ }^{1} \mathrm{H}$ and ${ }^{13} \mathrm{C}$ NMR spectra of 1d. ${ }^{1} \mathrm{H}$ NMR $\left(500 \mathrm{MHz}, \mathrm{CDCl}_{3}\right) \delta 7.39-7.30(\mathrm{~m}, 20 \mathrm{H})$, $5.03(\mathrm{~s}, 8 \mathrm{H}) .{ }^{13} \mathrm{C}$ NMR $\left(126 \mathrm{MHz}, \mathrm{CDCl}_{3}\right): \delta 206.76,135.27,128.94,128.18,128.04,54.08$. *denotes residual $\mathrm{H}_{2} \mathrm{O}$. 

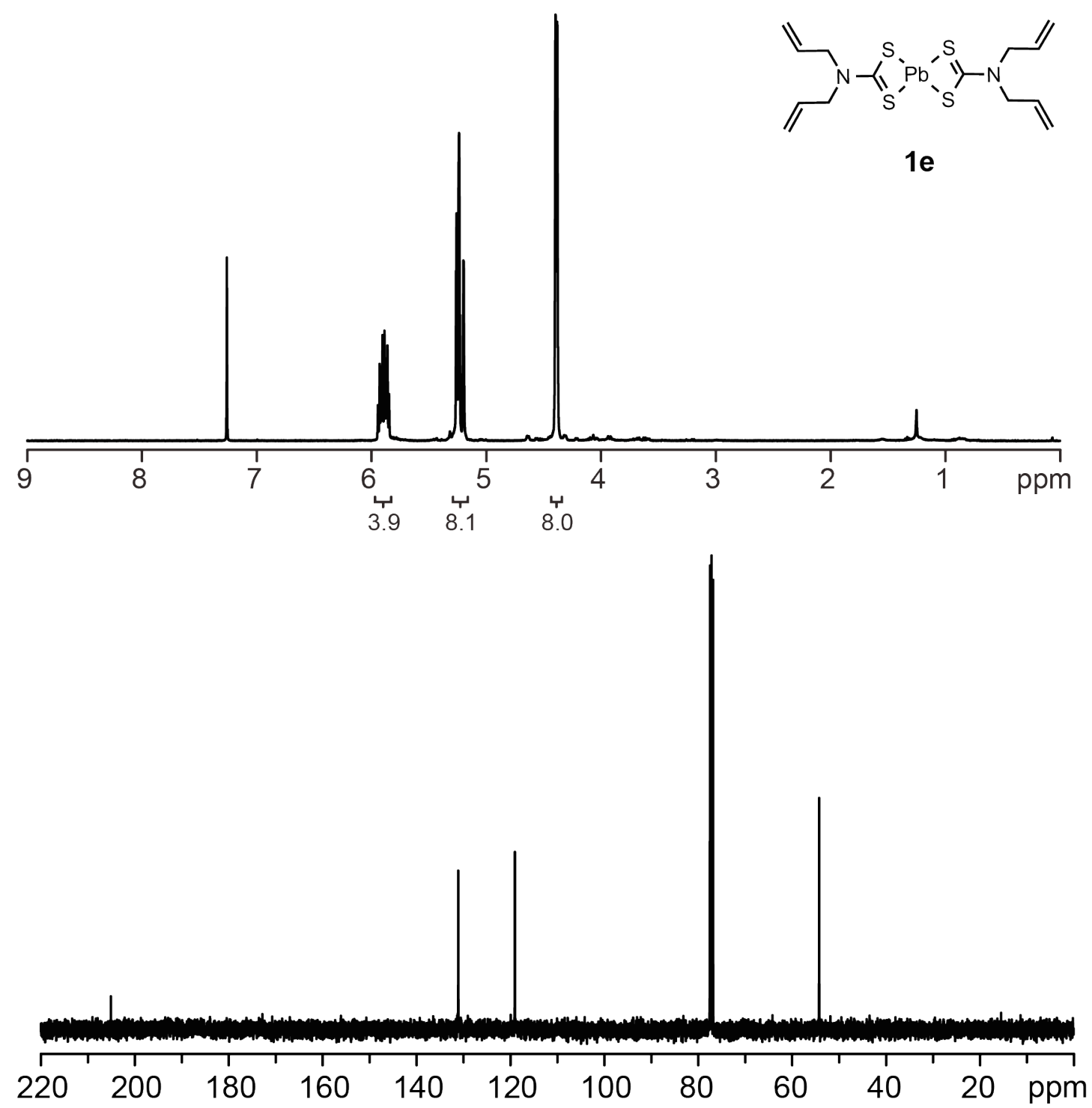

Figure S8. ${ }^{1} \mathrm{H}$ and ${ }^{13} \mathrm{C}$ NMR spectra of 1e. ${ }^{1} \mathrm{H}$ NMR $\left(400 \mathrm{MHz}, \mathrm{CDCl}_{3}\right) \delta 5.89$ (ddt, $J=16.4$, 10.3, $5.9 \mathrm{~Hz}, 4 \mathrm{H}), 5.28-5.17(\mathrm{~m}, 8 \mathrm{H}), 4.39(\mathrm{~d}, J=5.8 \mathrm{~Hz}, 8 \mathrm{H}) .{ }^{13} \mathrm{C} \mathrm{NMR}\left(101 \mathrm{MHz}, \mathrm{CDCl}_{3}\right): \delta$ $205.08,131.10,119.03,54.25$. 

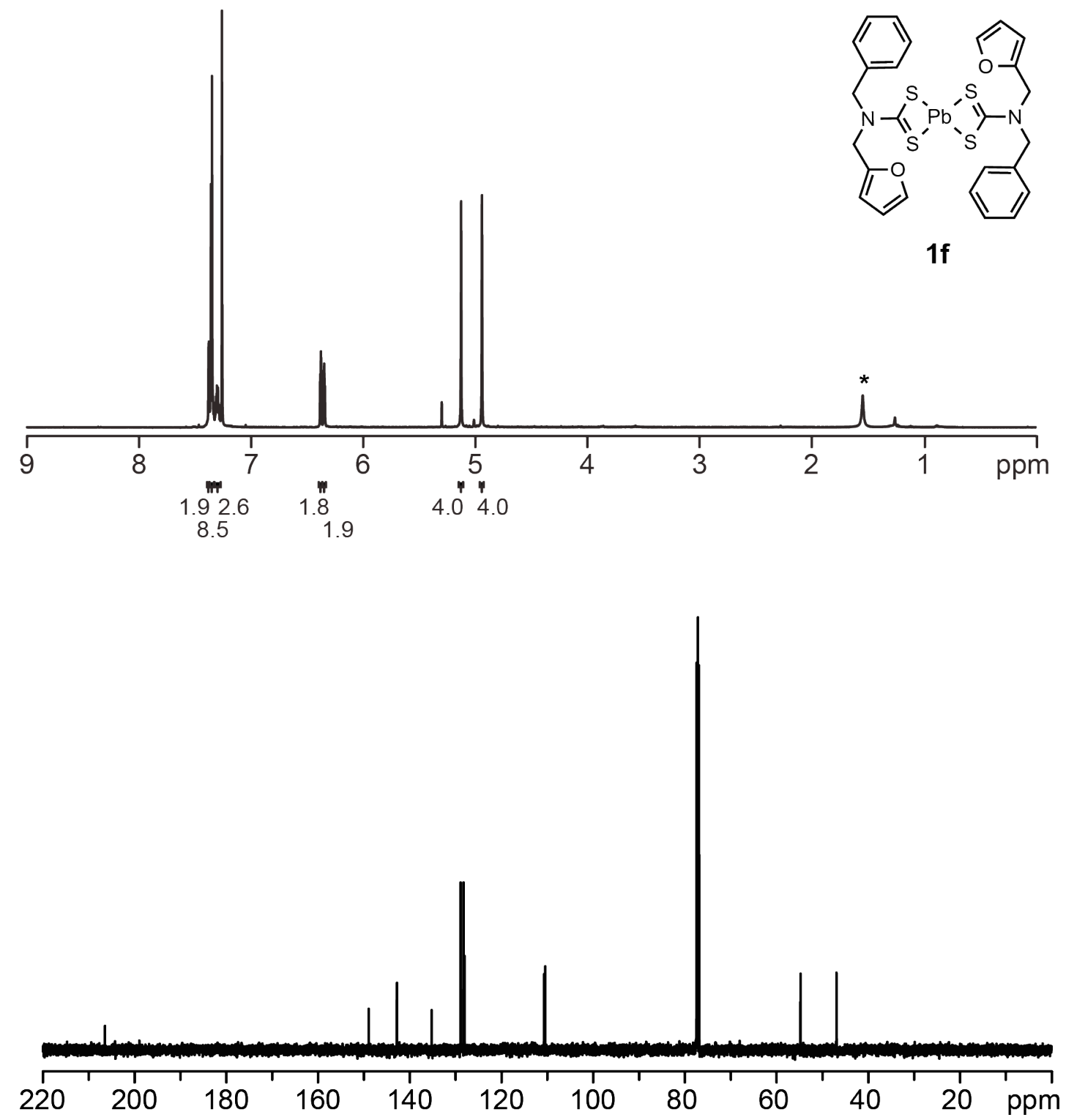

Figure S9. ${ }^{1} \mathrm{H}$ and ${ }^{13} \mathrm{C}$ NMR spectra of 1f. ${ }^{1} \mathrm{H}$ NMR $\left(500 \mathrm{MHz}, \mathrm{CDCl}_{3}\right) \delta 7.38(\mathrm{dd}, \mathrm{J}=1.8,0.8$ $\mathrm{Hz}, 2 \mathrm{H}), 7.35(\mathrm{~d}, J=4.4 \mathrm{~Hz}, 8 \mathrm{H}), 7.34-7.26(\mathrm{~m}, 2 \mathrm{H}), 6.38(\mathrm{~d}, J=3.2 \mathrm{~Hz}, 2 \mathrm{H}), 6.35(\mathrm{dd}, J=3.3$, $1.9 \mathrm{~Hz}, 2 \mathrm{H}), 5.13$ (s, 4H), 4.94 (s, 4H). ${ }^{13} \mathrm{C}$ NMR (126 MHz, $\left.\mathrm{CDCl}_{3}\right): \delta$ 206.43, 148.93, 142.77, 135.22, 128.89, 128.25, 128.04, 110.66, 110.44, 54.78, 46.94. *denotes residual $\mathrm{H}_{2} \mathrm{O}$. 

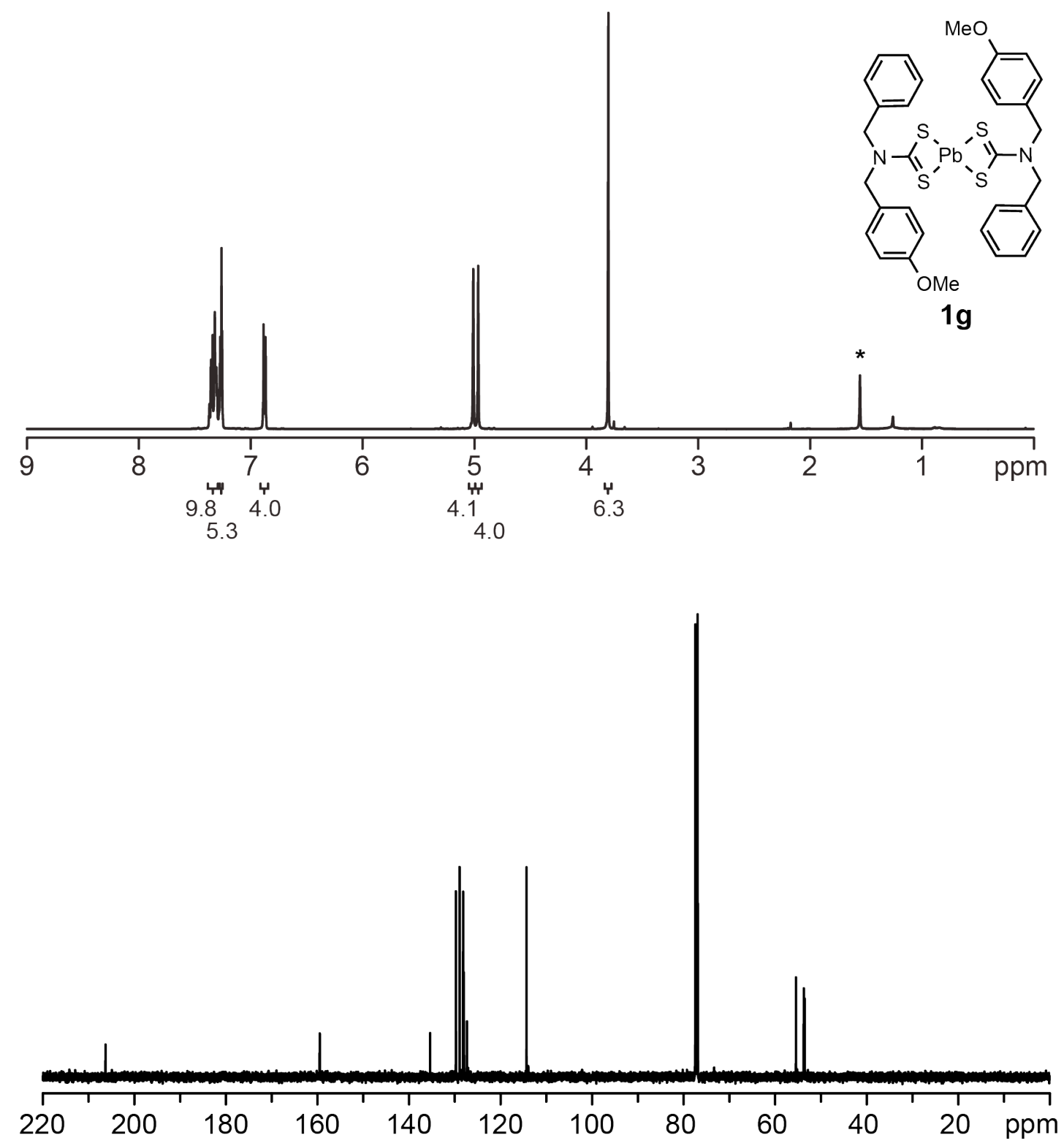

Figure S10. ${ }^{1} \mathrm{H}$ and ${ }^{13} \mathrm{C}$ NMR spectra of $1 \mathrm{~g} .{ }^{1} \mathrm{H}$ NMR $\left(500 \mathrm{MHz}, \mathrm{CDCl}_{3}\right) \delta 7.38-7.29(\mathrm{~m}, 10 \mathrm{H})$, $7.29-7.24(\mathrm{~m}, 4 \mathrm{H}), 6.90-6.85(\mathrm{~m}, 4 \mathrm{H}), 5.01(\mathrm{~s}, 4 \mathrm{H}), 4.97(\mathrm{~s}, 4 \mathrm{H}), 3.80(\mathrm{~s}, 6 \mathrm{H}) .{ }^{13} \mathrm{C}$ NMR $(126$ $\mathrm{MHz}_{\mathrm{CDCl}}$ ): $\delta$ 206.29, 159.48, 135.36, 129.73, 128.92, 128.16, 127.98, 127.31, 114.30, 55.45, 53.74, 53.55. *denotes residual $\mathrm{H}_{2} \mathrm{O}$. 


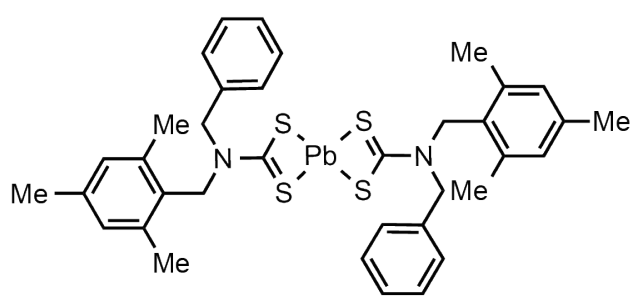

$1 \mathrm{~h}$
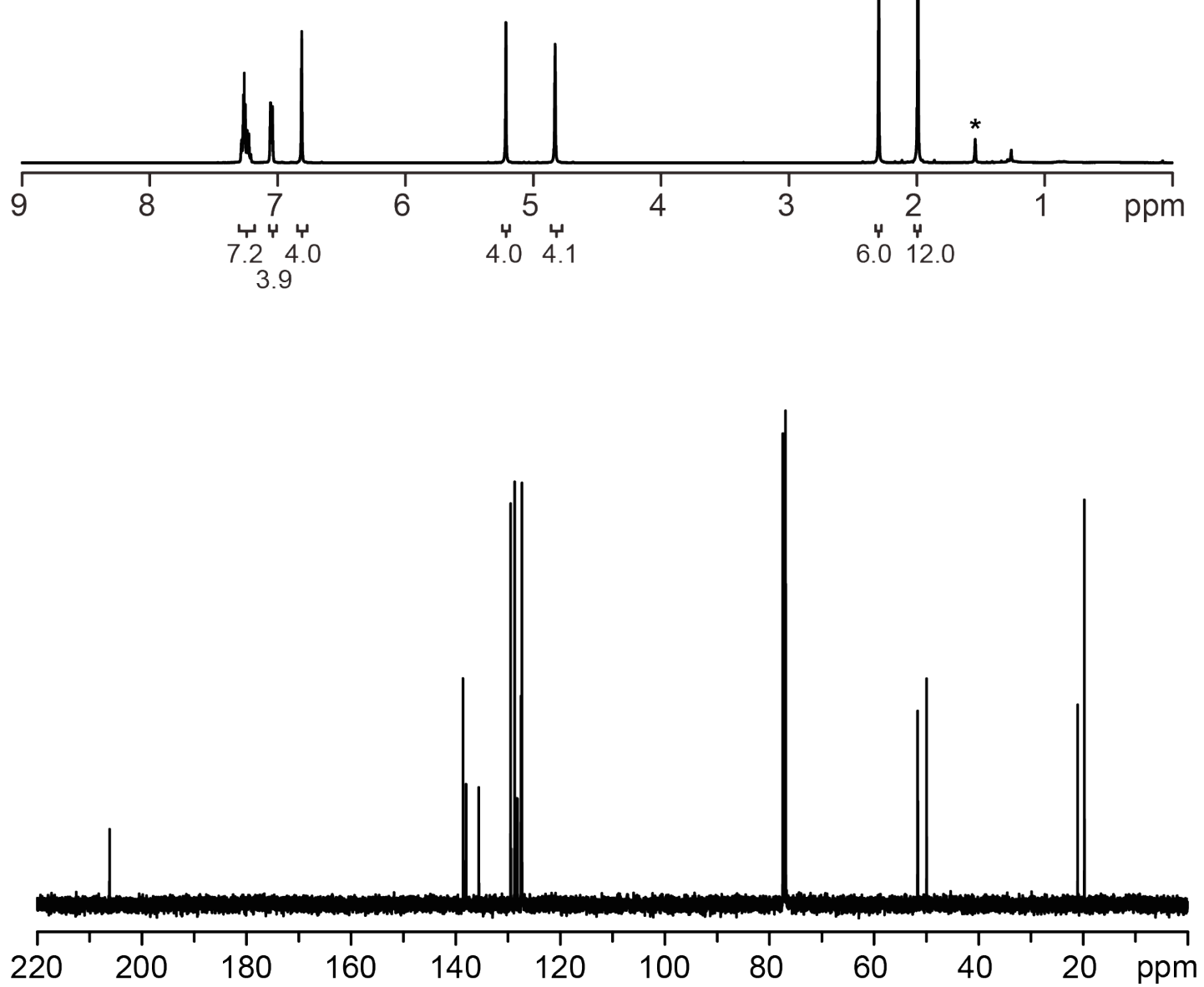

Figure S11. ${ }^{1} \mathrm{H}$ and ${ }^{13} \mathrm{C}$ NMR spectra of $1 \mathrm{~h} .{ }^{1} \mathrm{H}$ NMR $\left(500 \mathrm{MHz}, \mathrm{CDCl}_{3}\right) \delta 7.30-7.20(\mathrm{~m}, 6 \mathrm{H})$, $7.08-7.02(\mathrm{~m}, 4 \mathrm{H}), 6.81(\mathrm{~s}, 4 \mathrm{H}), 5.21(\mathrm{~s}, 4 \mathrm{H}), 4.83(\mathrm{~s}, 4 \mathrm{H}), 2.30(\mathrm{~s}, 6 \mathrm{H}), 1.99(\mathrm{~s}, 12 \mathrm{H}) .{ }^{13} \mathrm{C}$ NMR $\left(126 \mathrm{MHz}_{\mathrm{CDCl}}\right.$ ): $\delta$ 206.17, 138.60, 137.99, 135.57, 129.47, 128.69, 128.20, 127.48 , 127.31, 51.65, 49.94, 21.06, 19.77. *denotes residual $\mathrm{H}_{2} \mathrm{O}$. 

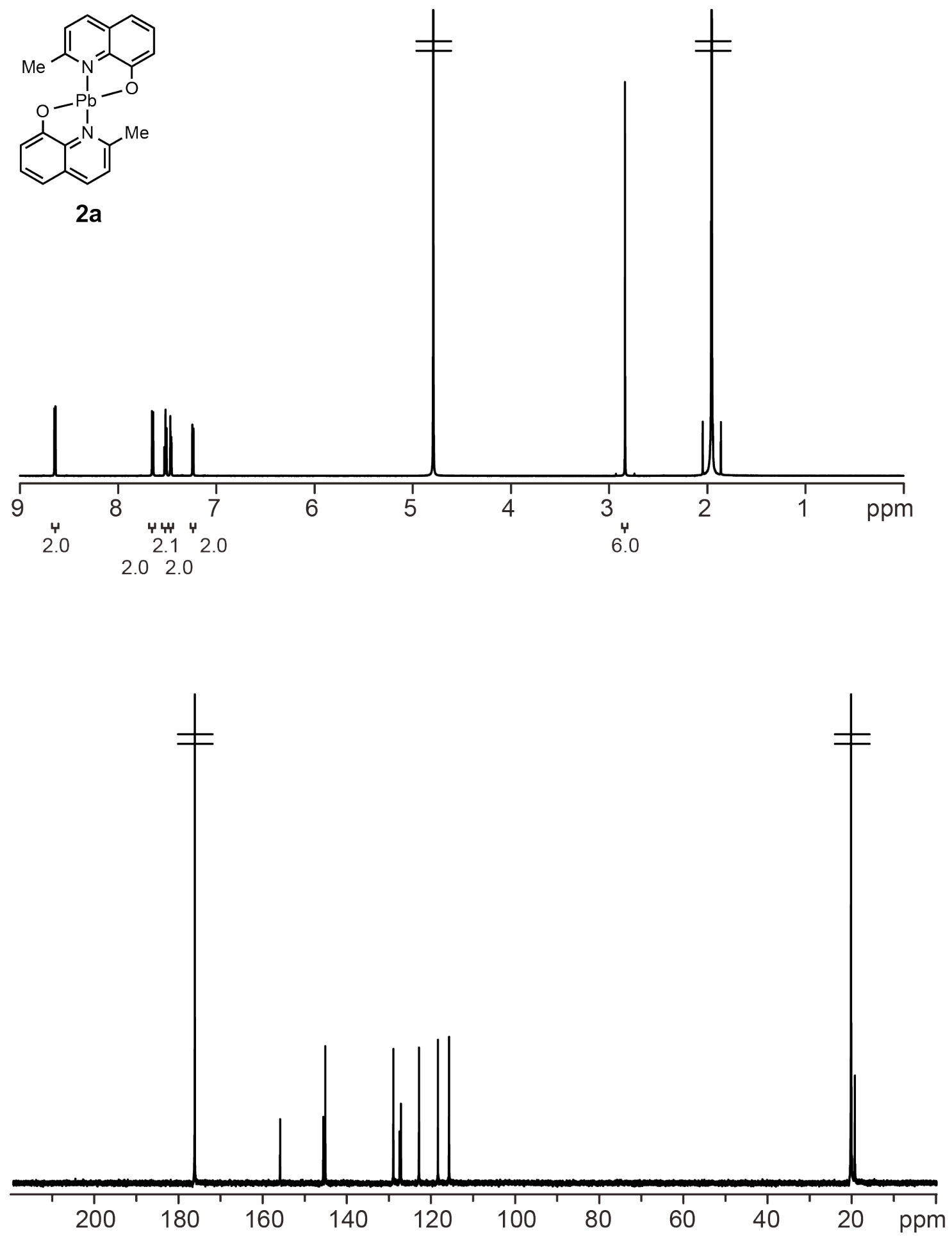

Figure S12. ${ }^{1} \mathrm{H}$ and ${ }^{13} \mathrm{C}$ NMR spectra of $2 \mathrm{a} .{ }^{1} \mathrm{H}$ NMR $\left(700 \mathrm{MHz}, \mathrm{D}_{2} \mathrm{O}\right.$ and DOAc) $\delta 8.50(\mathrm{~d}, \mathrm{~J}=8$ $\mathrm{Hz}, 2 \mathrm{H}), 7.50(\mathrm{~d}, J=8 \mathrm{~Hz}, 2 \mathrm{H}), 7.37(\mathrm{t}, J=8 \mathrm{~Hz}, 2 \mathrm{H}), 7.32(\mathrm{~d}, J=8 \mathrm{~Hz}, 2 \mathrm{H}), 7.09$ (d, J = $8 \mathrm{~Hz}$, $2 \mathrm{H}), 2.69(\mathrm{~s}, 6 \mathrm{H}) .{ }^{13} \mathrm{C}$ NMR $\left(176 \mathrm{MHz}, \mathrm{D}_{2} \mathrm{O}\right.$ and DOAc) $\delta$ 156.56, 146.31, 145.82, 129.60, $128.13,127.80,123.50,118.98,116.33,19.65$. 


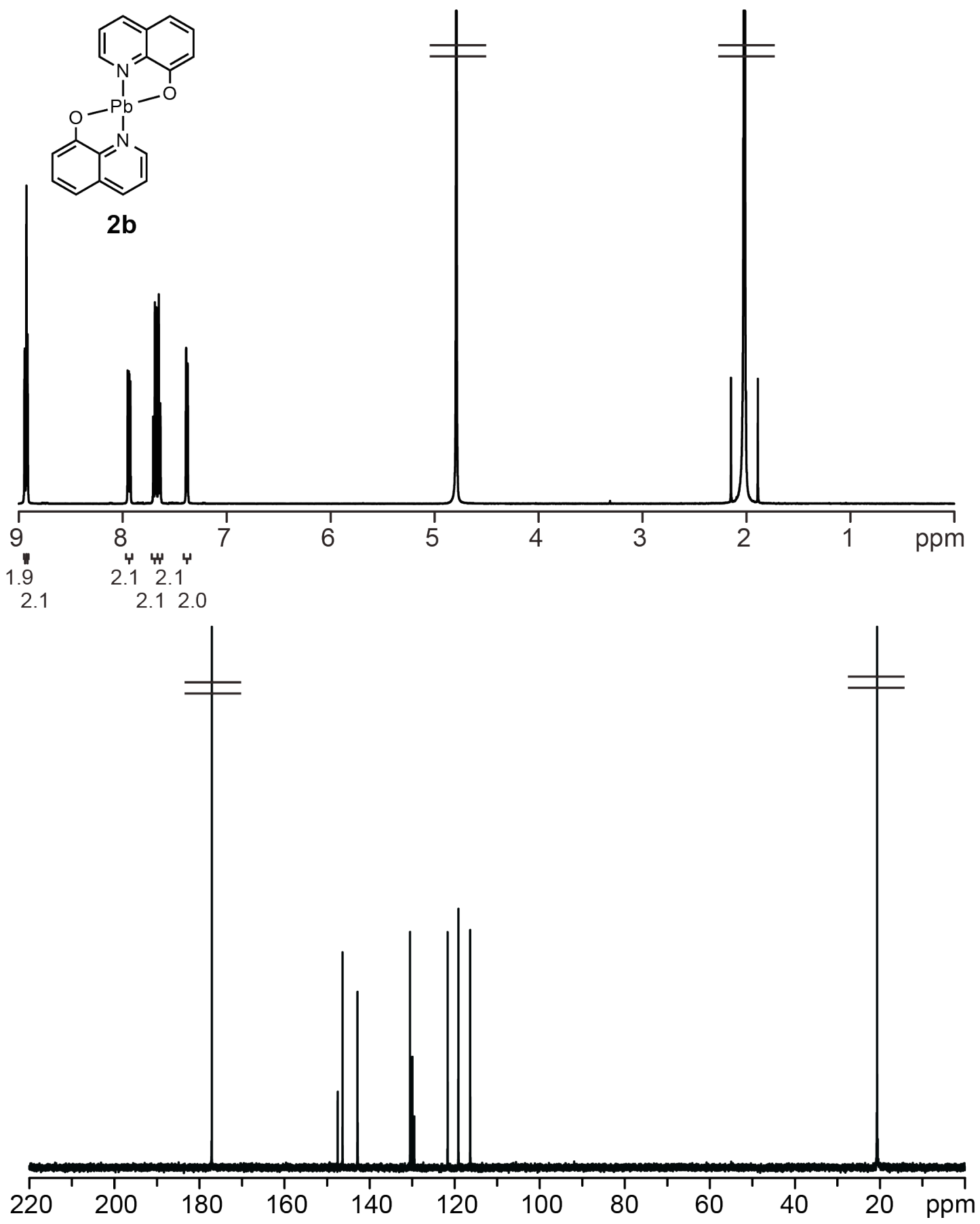

Figure S13. ${ }^{1} \mathrm{H}$ and ${ }^{13} \mathrm{C}$ NMR spectra of $2 \mathrm{~b} .{ }^{1} \mathrm{H}$ NMR $\left(700 \mathrm{MHz}, \mathrm{D}_{2} \mathrm{O}\right.$ and DOAc) $\delta 8.78(\mathrm{~d}, \mathrm{~J}=8$ $\mathrm{Hz}, 2 \mathrm{H}$ ), $8.76(\mathrm{~d}, J=5 \mathrm{~Hz}, 2 \mathrm{H}), 7.79(\mathrm{dd}, J=8,5 \mathrm{~Hz}, 2 \mathrm{H}), 7.52$ (dd, $J=8,8 \mathrm{~Hz}, 2 \mathrm{H}), 7.48(\mathrm{~d}, J$ $=8 \mathrm{~Hz}, 2 \mathrm{H}), 7.22(\mathrm{~d}, J=8 \mathrm{~Hz}, 2 \mathrm{H}) .{ }^{13} \mathrm{C} \mathrm{NMR}\left(176 \mathrm{MHz}, \mathrm{D}_{2} \mathrm{O}\right.$ and DOAc) $\delta$ 147.48, 146.36, $142.83,130.46,129.91,129.47,121.64,119.10,116.33$. 

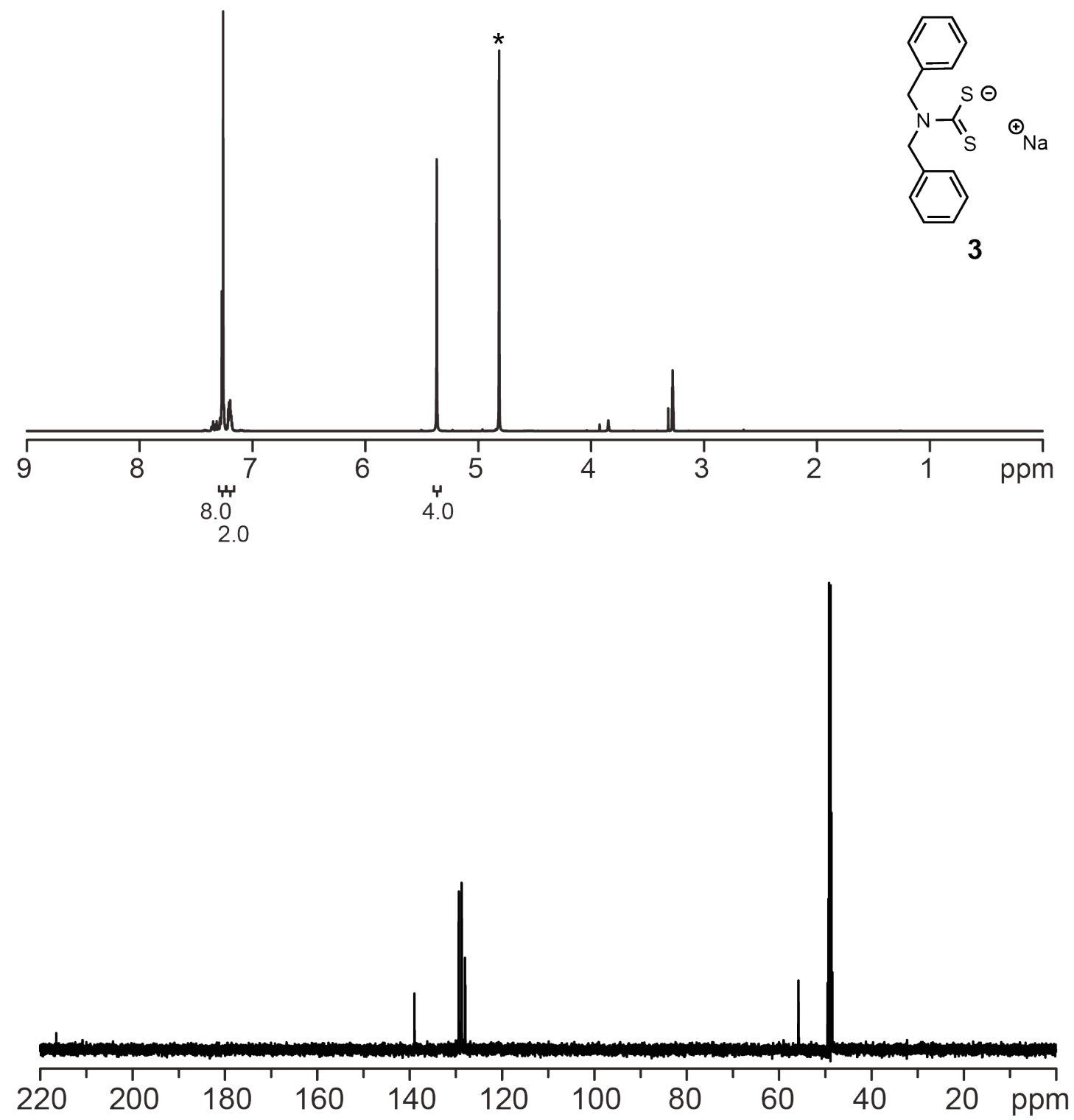

Figure S14. ${ }^{1} \mathrm{H}$ and ${ }^{13} \mathrm{C}$ NMR spectra of 3. ${ }^{1} \mathrm{H}$ NMR $\left(500 \mathrm{MHz}, \mathrm{CD}_{3} \mathrm{OD}\right) \delta 7.31-7.27(\mathrm{~m}, 8 \mathrm{H})$, $7.26-7.20(\mathrm{~m}, 2 \mathrm{H}), 5.40(\mathrm{~s}, 4 \mathrm{H}) .{ }^{13} \mathrm{C} \mathrm{NMR}\left(126 \mathrm{MHz}, \mathrm{CD}_{3} \mathrm{OD}\right): \delta 216.52,138.95,129.35$, 128.73, 127.98, 55.80. *denotes residual $\mathrm{H}_{2} \mathrm{O}$. 


\section{Cambridge Structural Database (CSD) Search Method}

\section{A search for Pb-complexes was performed using the Draw tool in CCDC Conquest.}

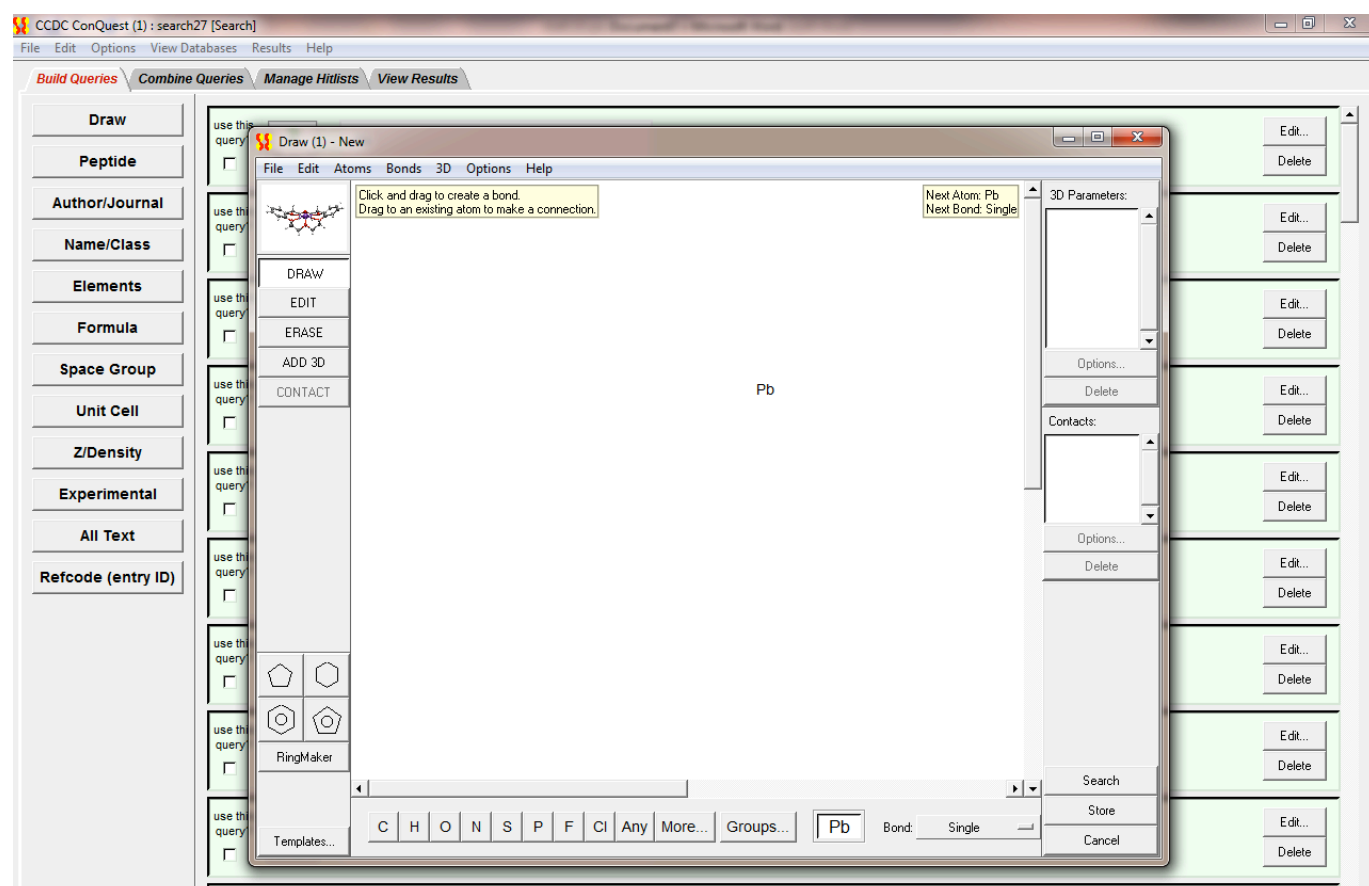

2. The following filters were applied to ensure that the CIF files were adequate for our morphology prediction calculations.

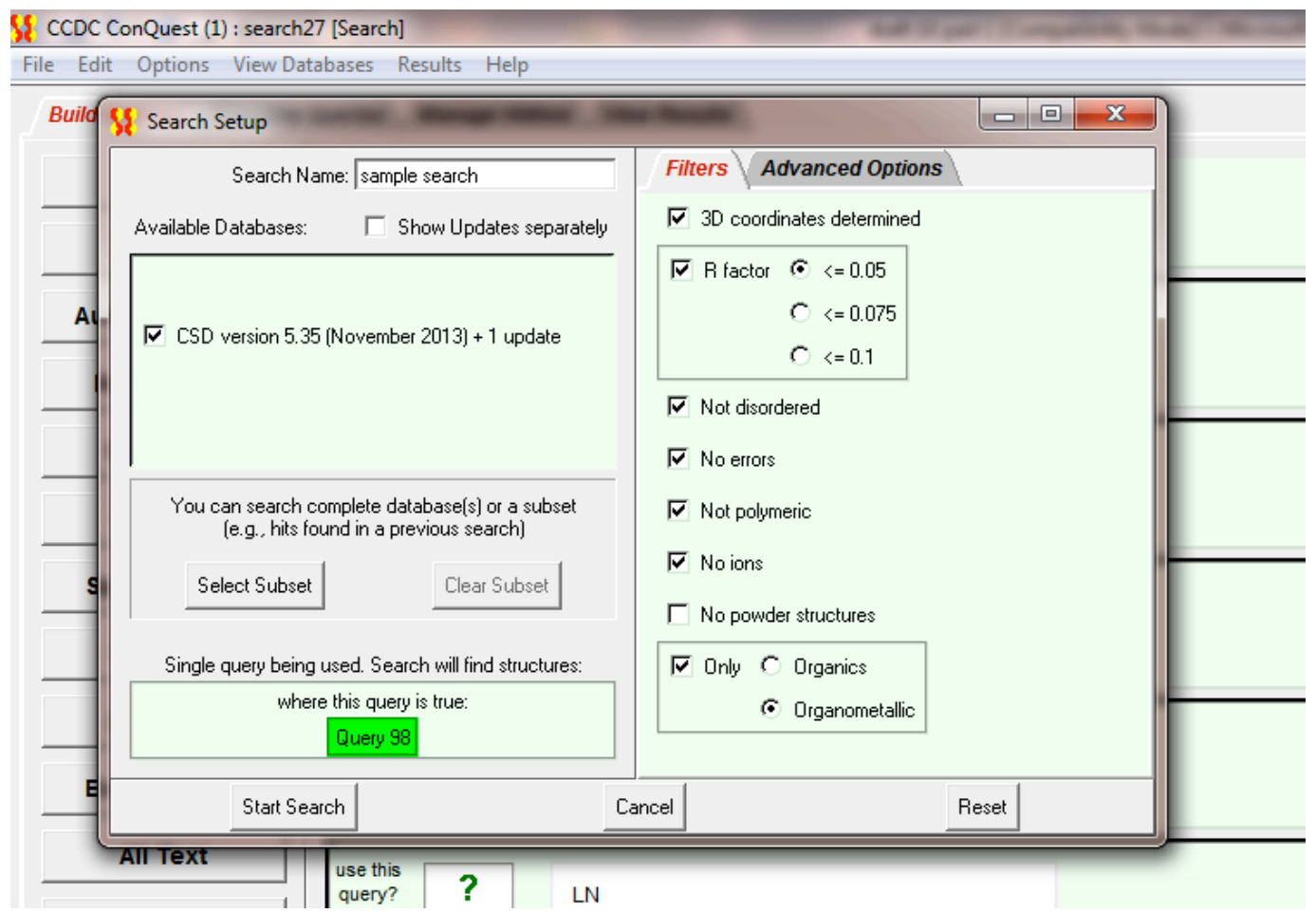


3. Using the Combine Queries in combination with the Draw tool, complexes containing all other metals and metalloids were excluded from the search.

\begin{tabular}{|c|c|c|c|c|c|c|}
\hline \multicolumn{7}{|c|}{ CCDC ConQuest (1) : sample search [Search] } \\
\hline \multicolumn{7}{|c|}{ File Edit Options View Databases Results Help } \\
\hline \multicolumn{3}{|c|}{ Build Queries Combine Queries } & Manage Hitlis & View Results & & \\
\hline \multicolumn{3}{|c|}{\begin{tabular}{|l} 
Drag Query Icons into Boxes \\
\end{tabular}} & \multirow{2}{*}{\multicolumn{2}{|c|}{\begin{tabular}{|c|} 
\\
Query 88 \\
\end{tabular}}} & Edt... & $\Delta$ \\
\hline \multicolumn{3}{|c|}{$\begin{array}{l}\text { Find entries that: } \\
\text { must have } \quad \text { (boolean AND) }\end{array}$} & & & Delete & \\
\hline \multirow{6}{*}{\multicolumn{3}{|c|}{$\begin{array}{c}? \\
\text { Query } 89 \\
\end{array}$}} & \multirow{2}{*}{\multicolumn{2}{|c|}{\begin{tabular}{|c|}
$?$ \\
Query 89 \\
\end{tabular}}} & Edt... & \\
\hline & & & & & Delete & \\
\hline & & & \multirow{2}{*}{\multicolumn{2}{|c|}{\begin{tabular}{|c|}
$?$ \\
Query 90 \\
\end{tabular}}} & Edit... & \\
\hline & & & & & Delete & \\
\hline & & & \multirow{2}{*}{\multicolumn{2}{|c|}{$\begin{array}{c}? \\
\text { Query } 91 \\
\end{array}$}} & Edit... & \\
\hline & & & & & Delete & \\
\hline \multicolumn{3}{|c|}{ must not have } & \multirow[b]{2}{*}{$\begin{array}{c}? \\
\text { Query } 92 \\
\end{array}$} & \multirow[b]{2}{*}{$3 \mathrm{~A}$} & Edit... & \\
\hline $\begin{array}{c}? \\
\text { Query } 90\end{array}$ & \begin{tabular}{|c|}
$?$ \\
Query 91 \\
\end{tabular} & \begin{tabular}{|c|} 
\\
Query 93 \\
\end{tabular} & & & Delete & \\
\hline \begin{tabular}{|c|}
$?$ \\
Query 94 \\
\end{tabular} & $\begin{array}{c}? \\
\text { Query } 95\end{array}$ & $\begin{array}{c}? \\
\text { Query } 96\end{array}$ & \multirow{2}{*}{$\begin{array}{c}? \\
\text { Query } 93 \\
\end{array}$} & \multirow[t]{2}{*}{ LN } & Edit... & \\
\hline \multirow{3}{*}{$\begin{array}{c}? \\
\text { Query } 97\end{array}$} & \multirow{3}{*}{\begin{tabular}{|c|}
$?$ \\
Query 92 \\
\end{tabular}} & \multirow{3}{*}{$\begin{array}{c}? \\
\text { Query } 98\end{array}$} & & & Delete & \\
\hline & & & $?$ & $\mathrm{si}$ & Edt... & \\
\hline & & & Query 94 & & Delete & \\
\hline \multirow{2}{*}{\multicolumn{3}{|c|}{ must have at least one of $(O R)$}} & \multirow{2}{*}{\begin{tabular}{c|}
$?$ \\
Query 95
\end{tabular}} & \multirow{2}{*}{ As } & Edt... & \\
\hline & & & & & Delete & \\
\hline
\end{tabular}

4. Solvate structures were excluded from the search manually.

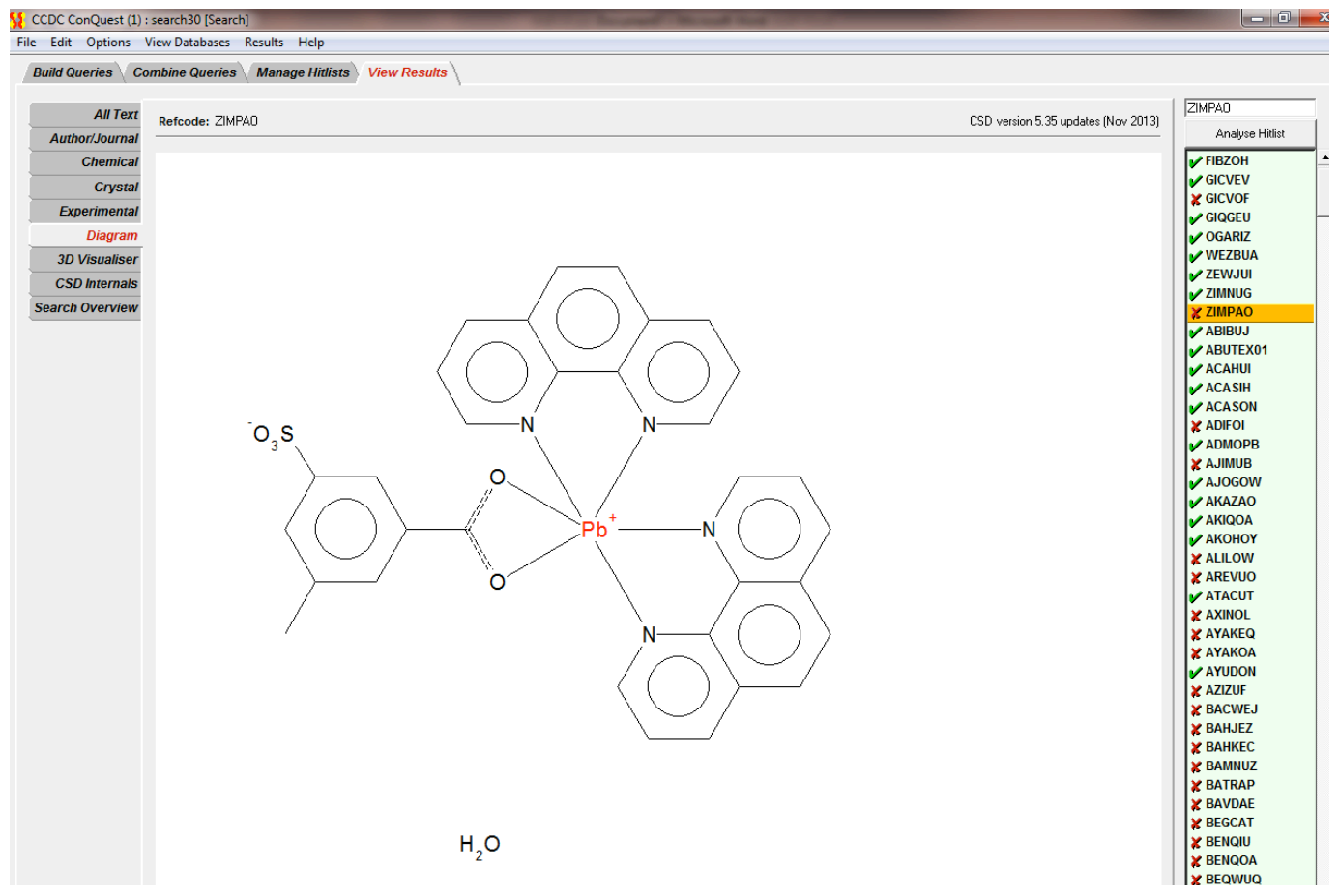




\section{Morphology Prediction Method}

1. CIF files were downloaded from the CSD and opened with Materials Studio using File $\rightarrow$ Import.

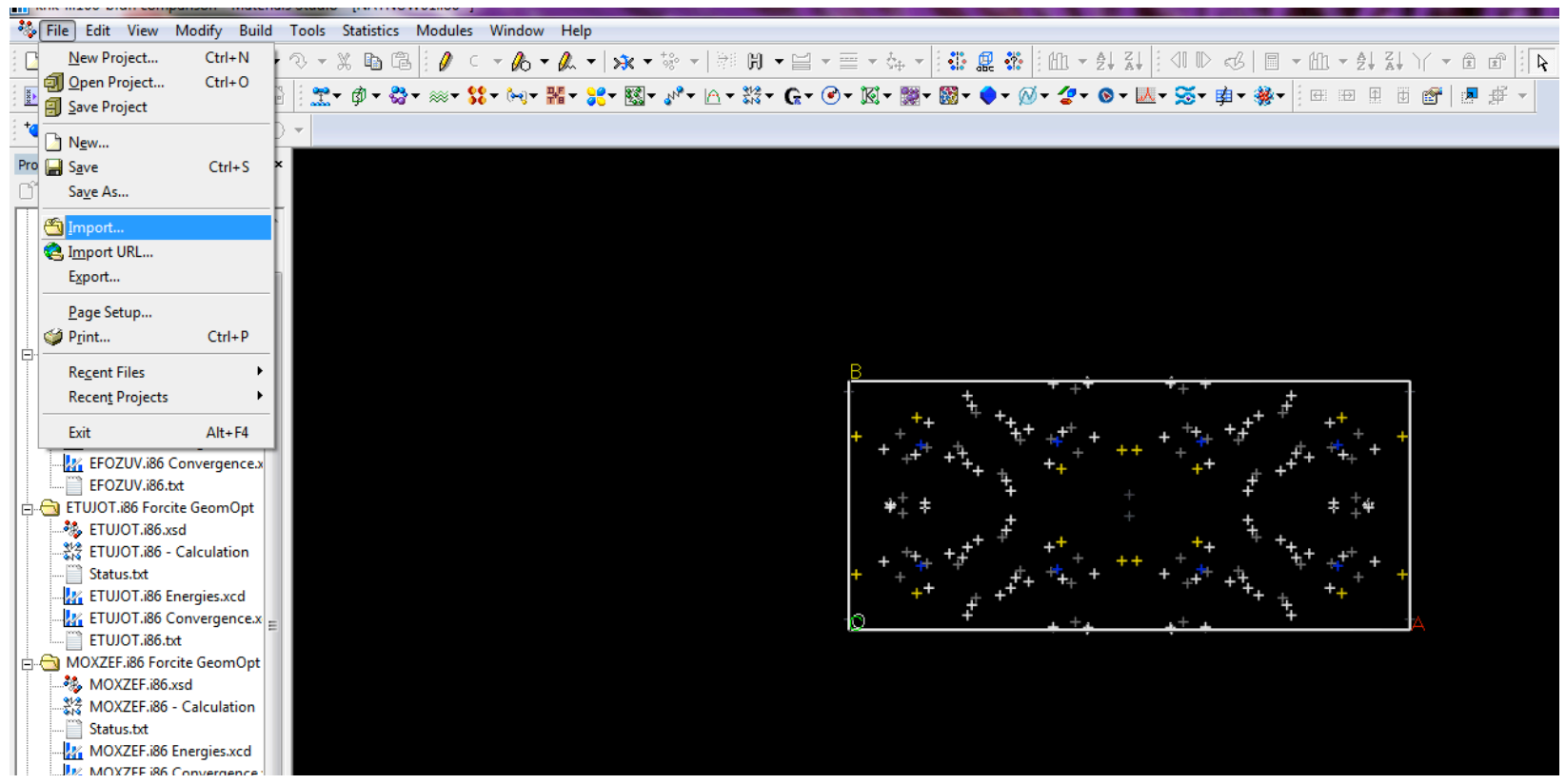

2. Bonds were calculated using the default settings for the distance between atoms and the atom type.

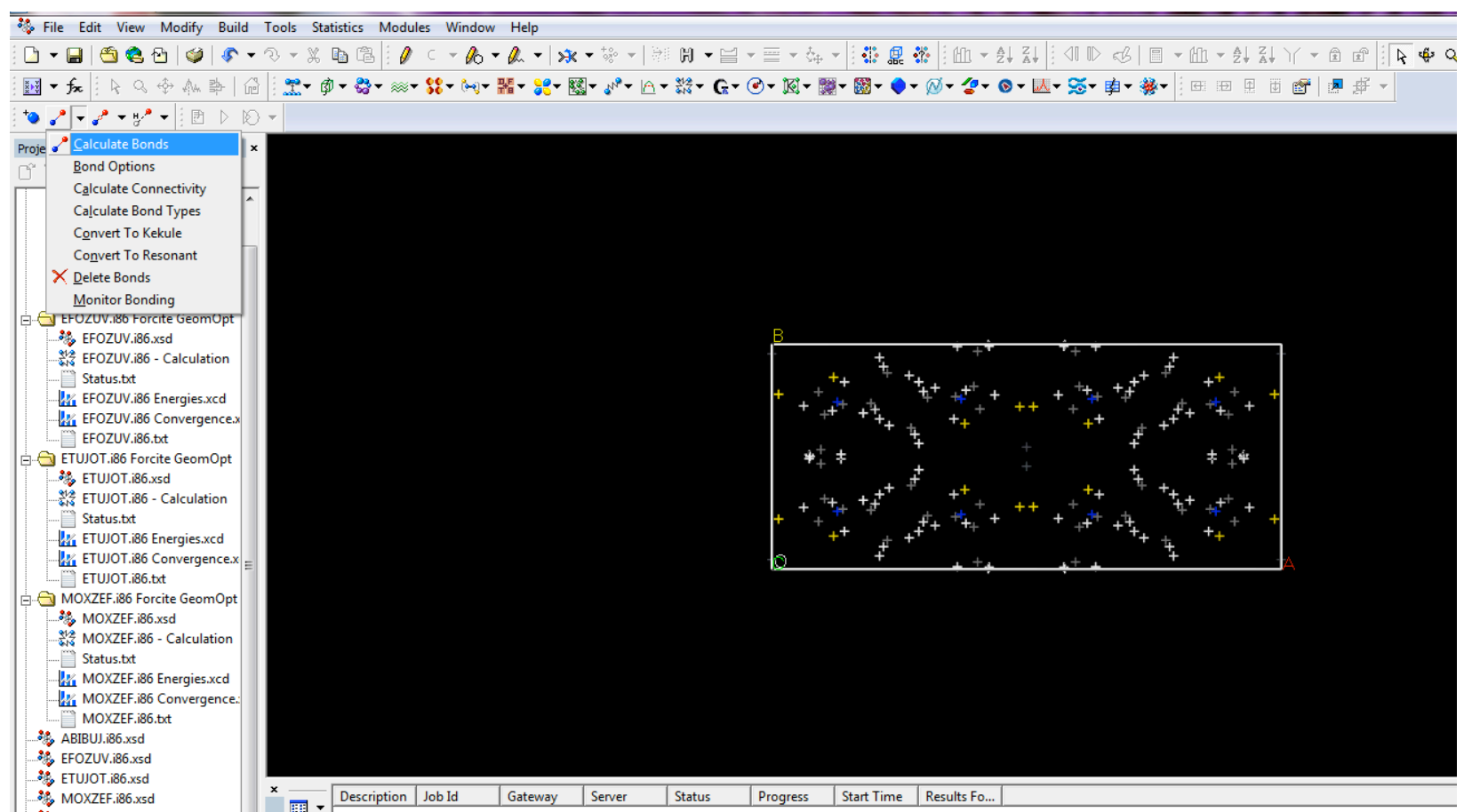


3. Molecular structures were completed using the Build Crystal tool.

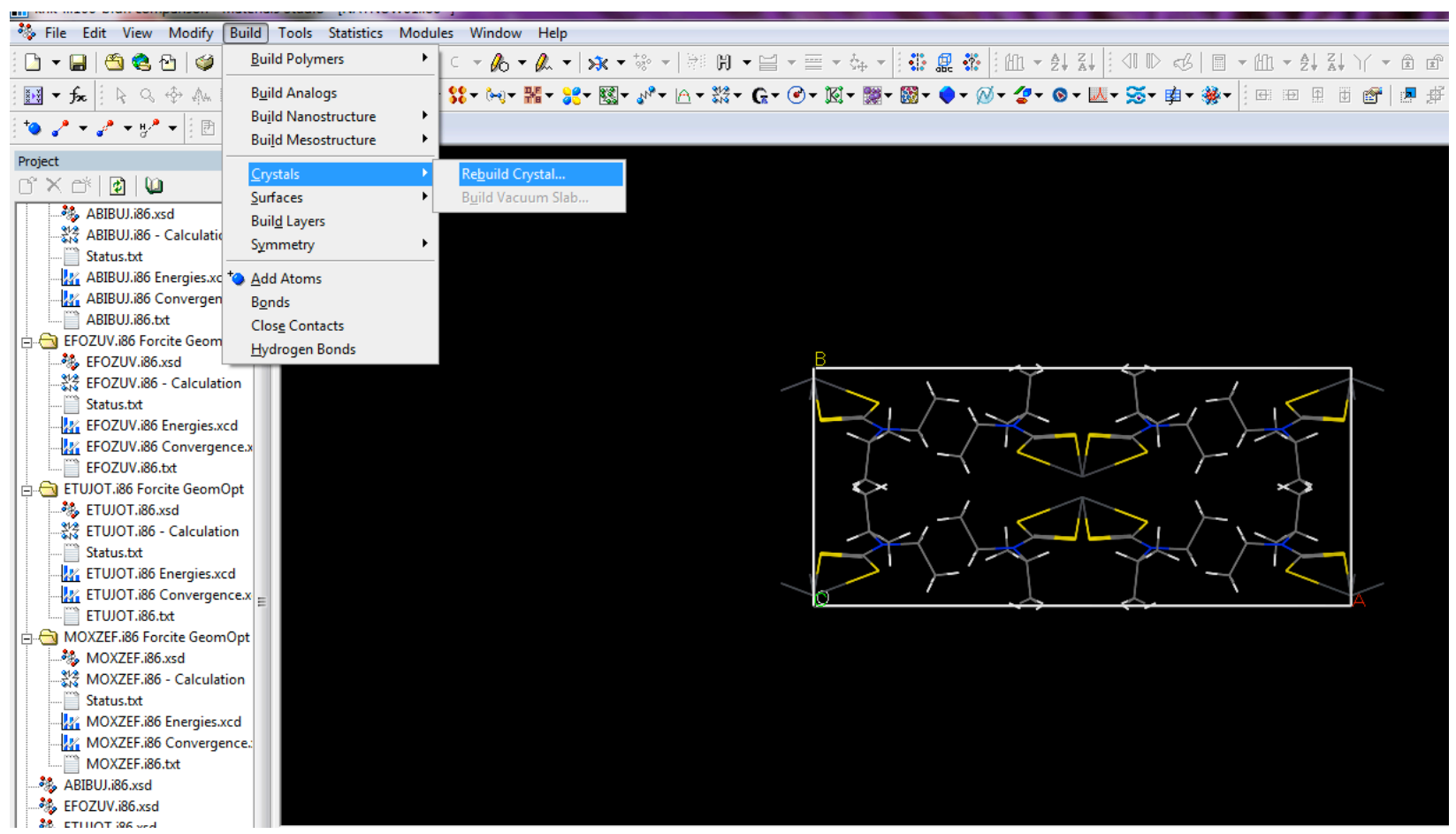

4. Hydrogen atom positions were normalized via Modify $\rightarrow$ Adjust Hydrogens.

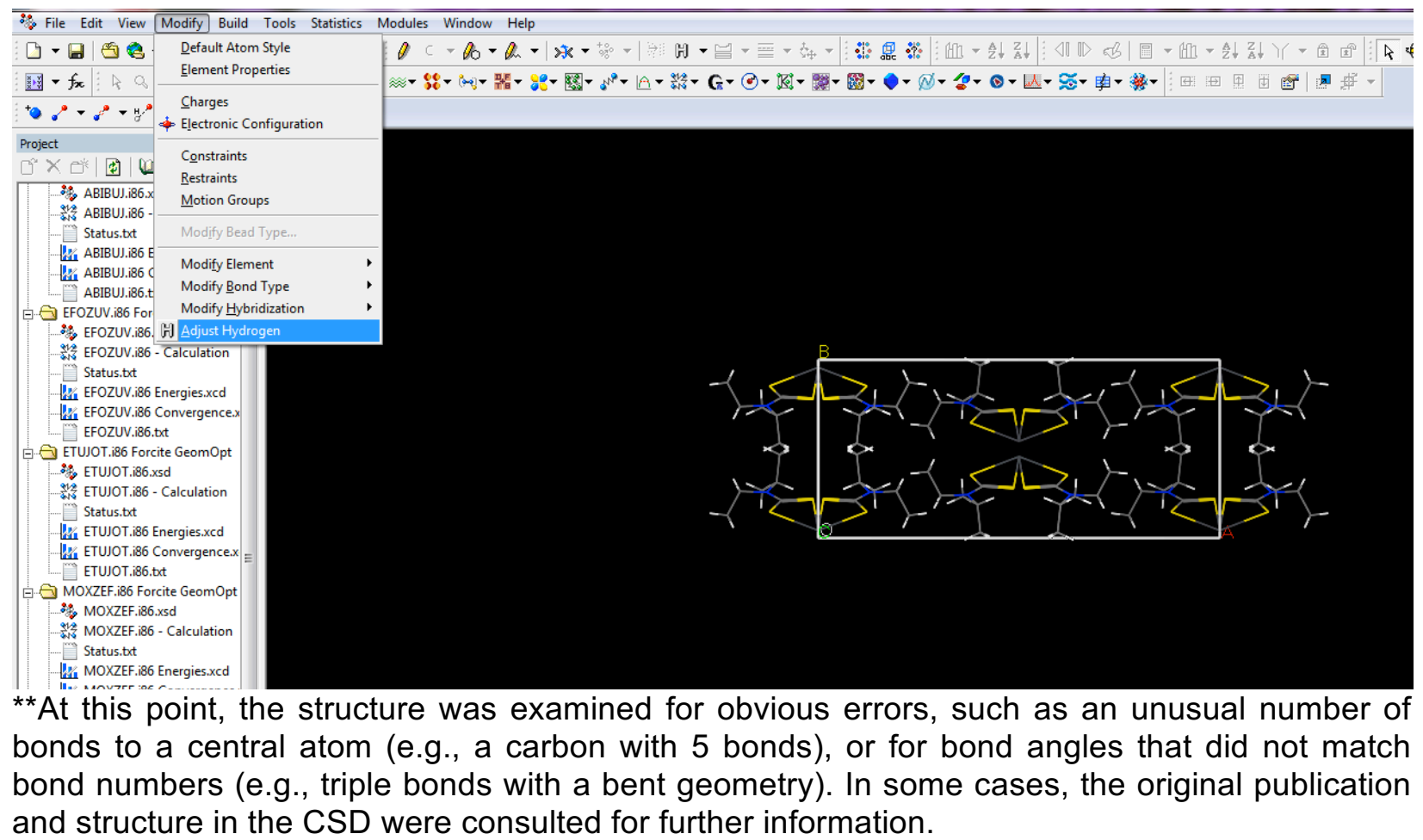


5. Geometry optimization was performed using Forcite. The Forcite module can be accessed by selecting Module $\rightarrow$ Forcite $\rightarrow$ Calculation.

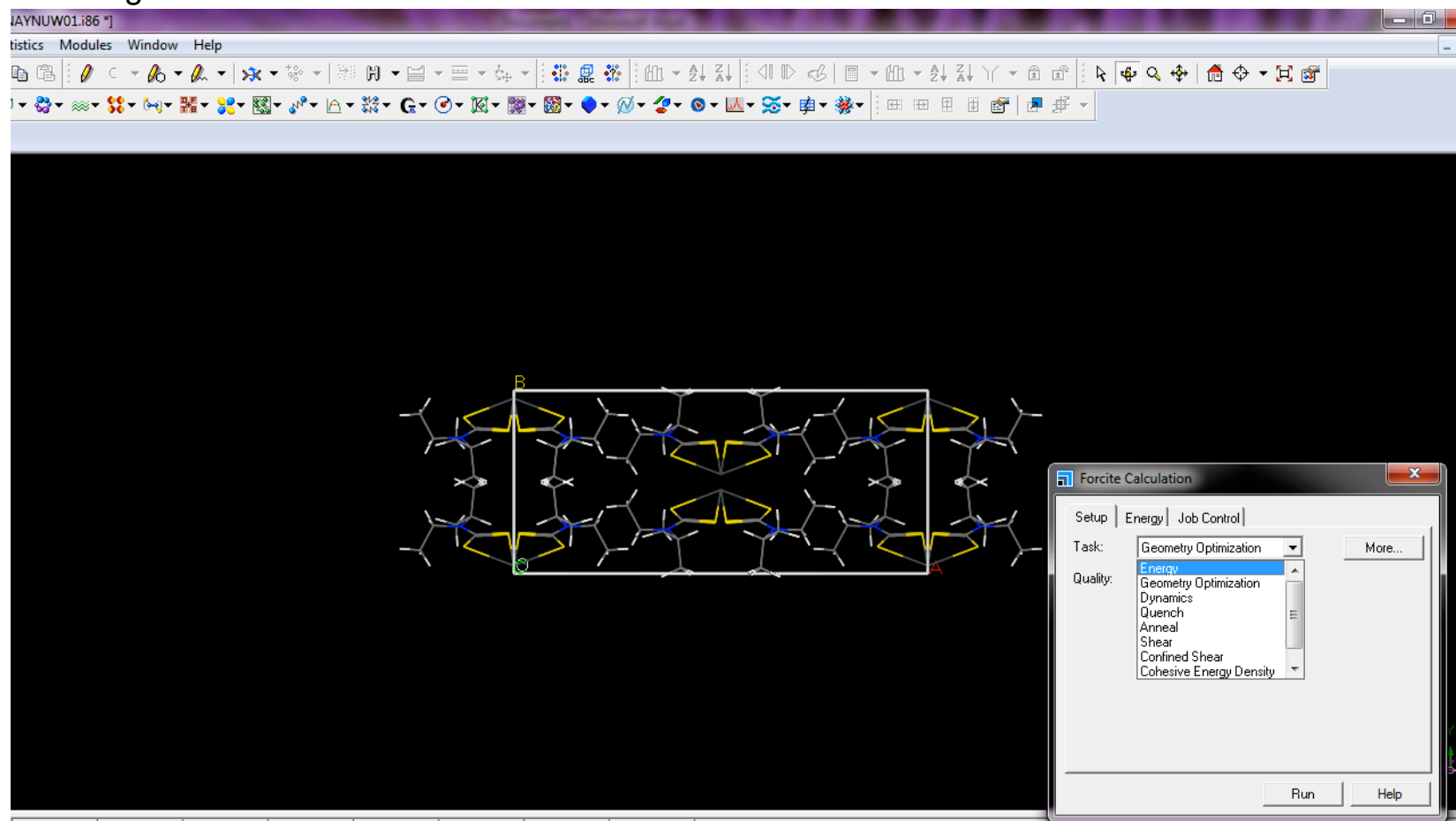

\begin{tabular}{|l|l|l|l|l|l|l|l|}
\hline Description & Job Id & Gateway & Server & Status & Progress & Start Time & Results Fo... \\
\hline
\end{tabular}

6. The "More..." button was selected to open a menu. Checking the box "optimize cell" leads to an unconstrained geometry optimization, where the unit cell parameters can change. For a constrained geometry optimization (no changes in the unit cell parameters), leave this box unchecked.

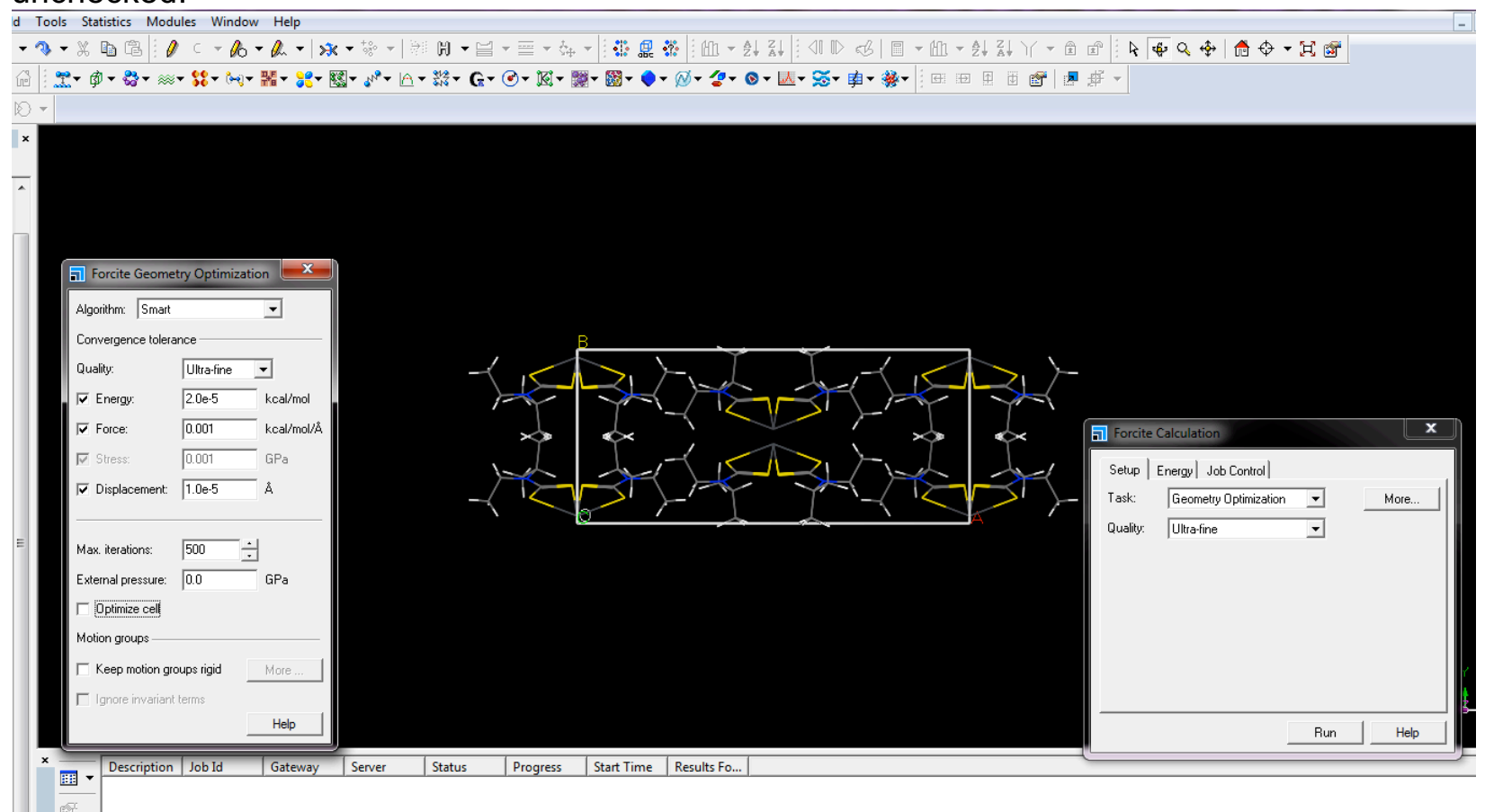


7. The calculation was performed at the ultra-fine quality level with the Universal Force Field (UFF) with charges assigned using QEq and electrostatic and van der Waals summation methods both set at Ewald.

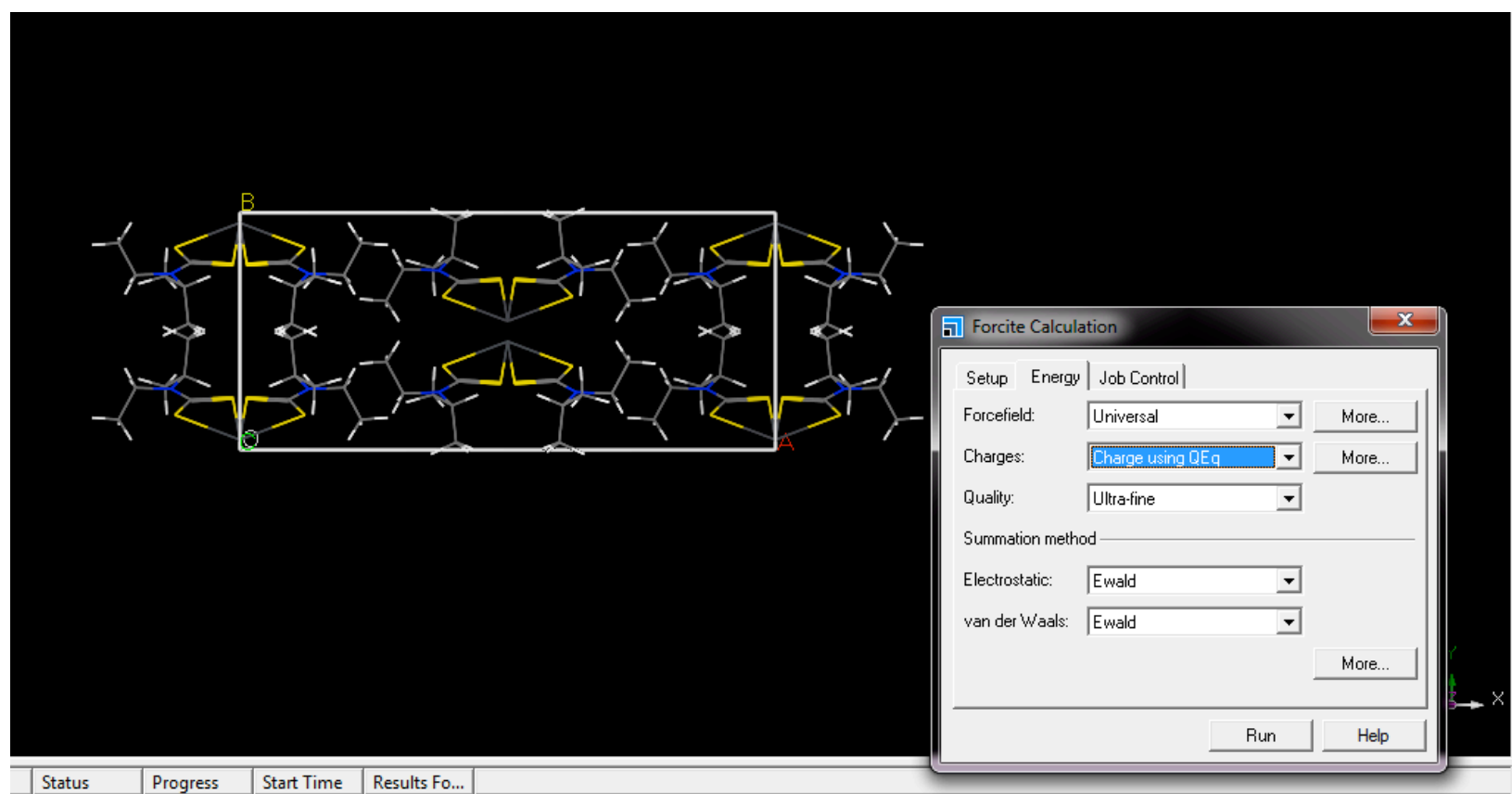

8. Crystal graphs were generated using the Module $\rightarrow$ Morphology $\rightarrow$ Crystal Graph.

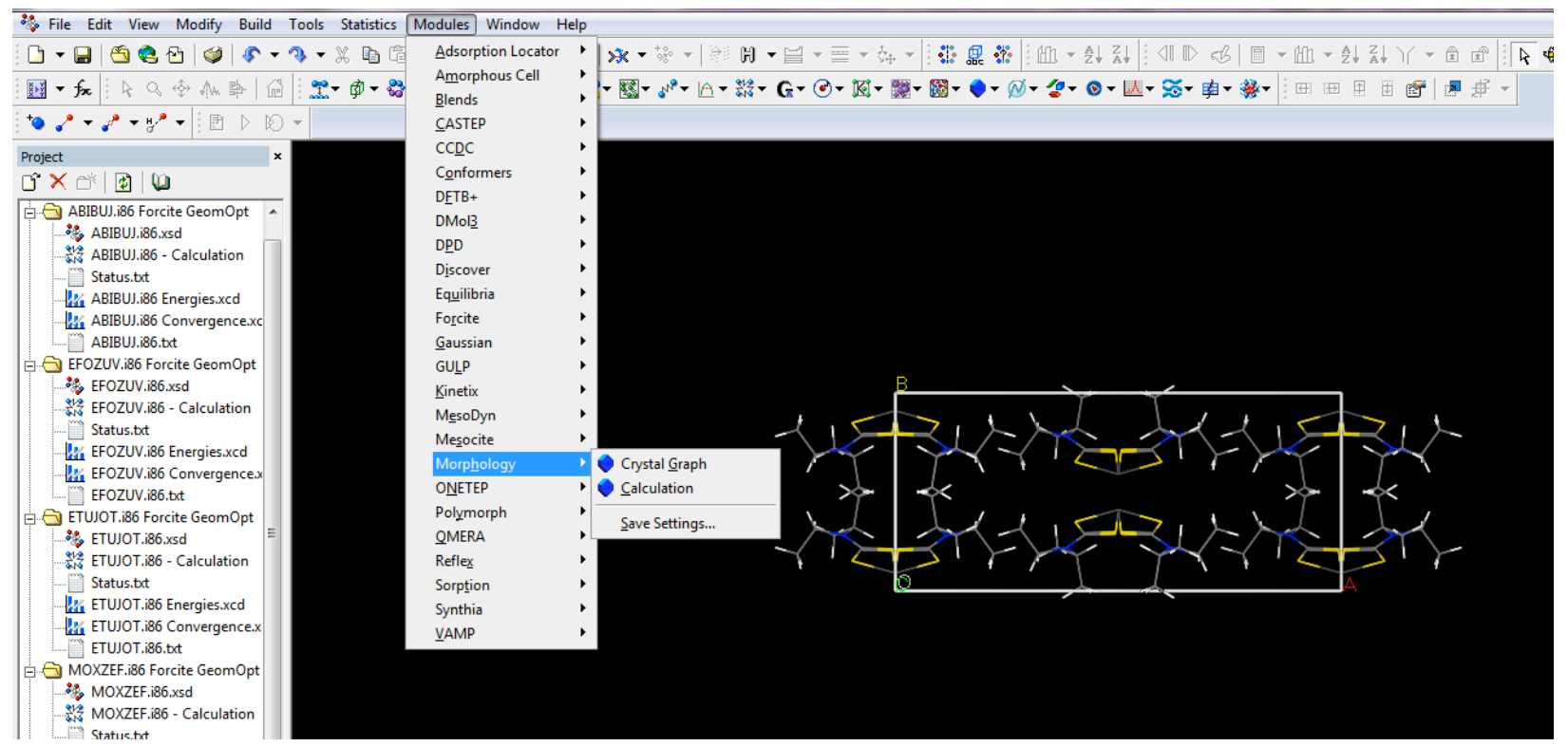


9. The crystal graph was generated using the same force field and charge assignment as the geometry optimization (see \#7 above). The maximum unit cell separation was set to one by one by one (calculation sphere), which can be done by either entering in the numbers or by selecting a "coarse" quality setting. A minimum energy of interaction filter was set to $-0.596 \mathrm{kcal} / \mathrm{mol}$.

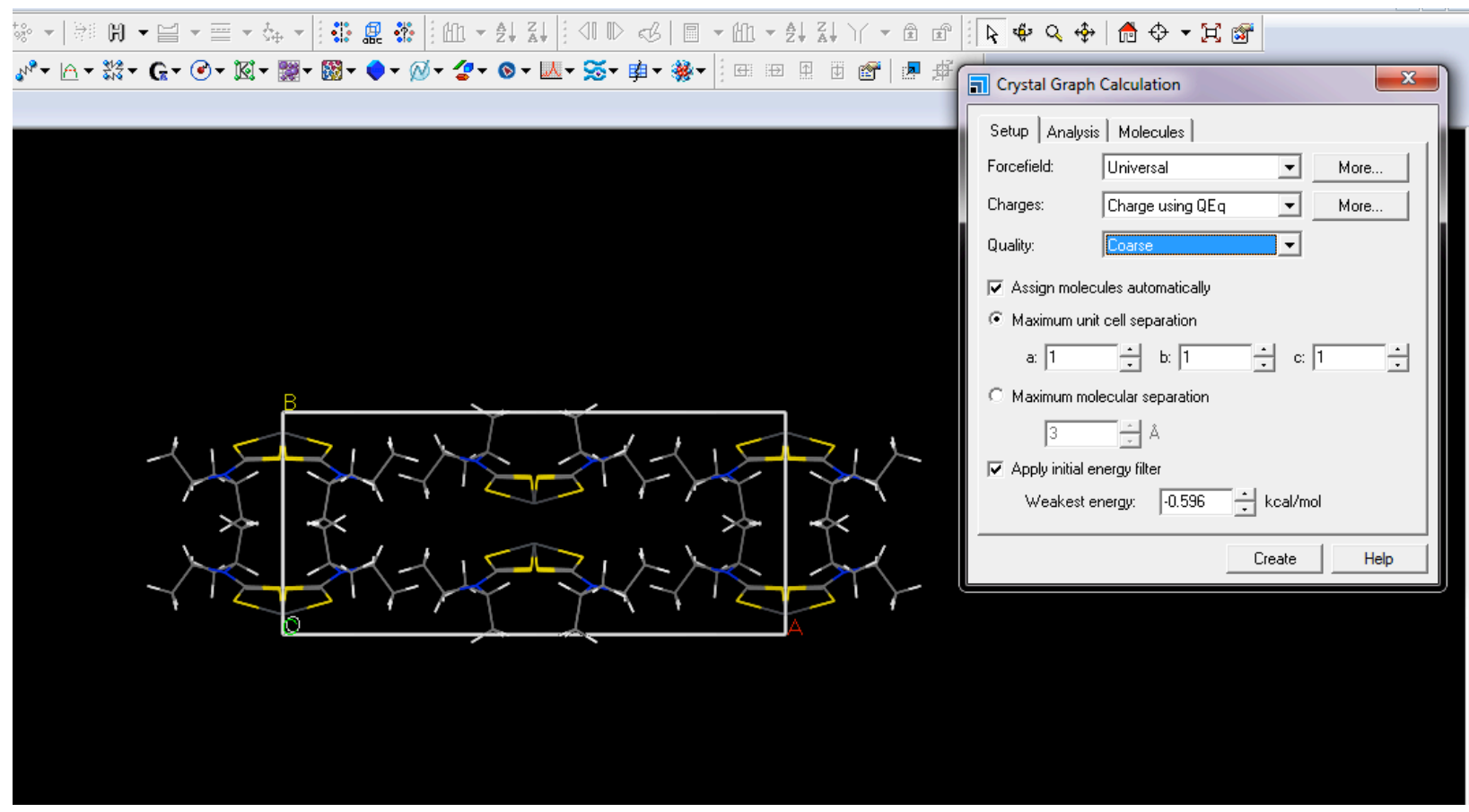

10. Morphologies were predicted by selecting Module $\rightarrow$ Morphology $\rightarrow$ Calculation.

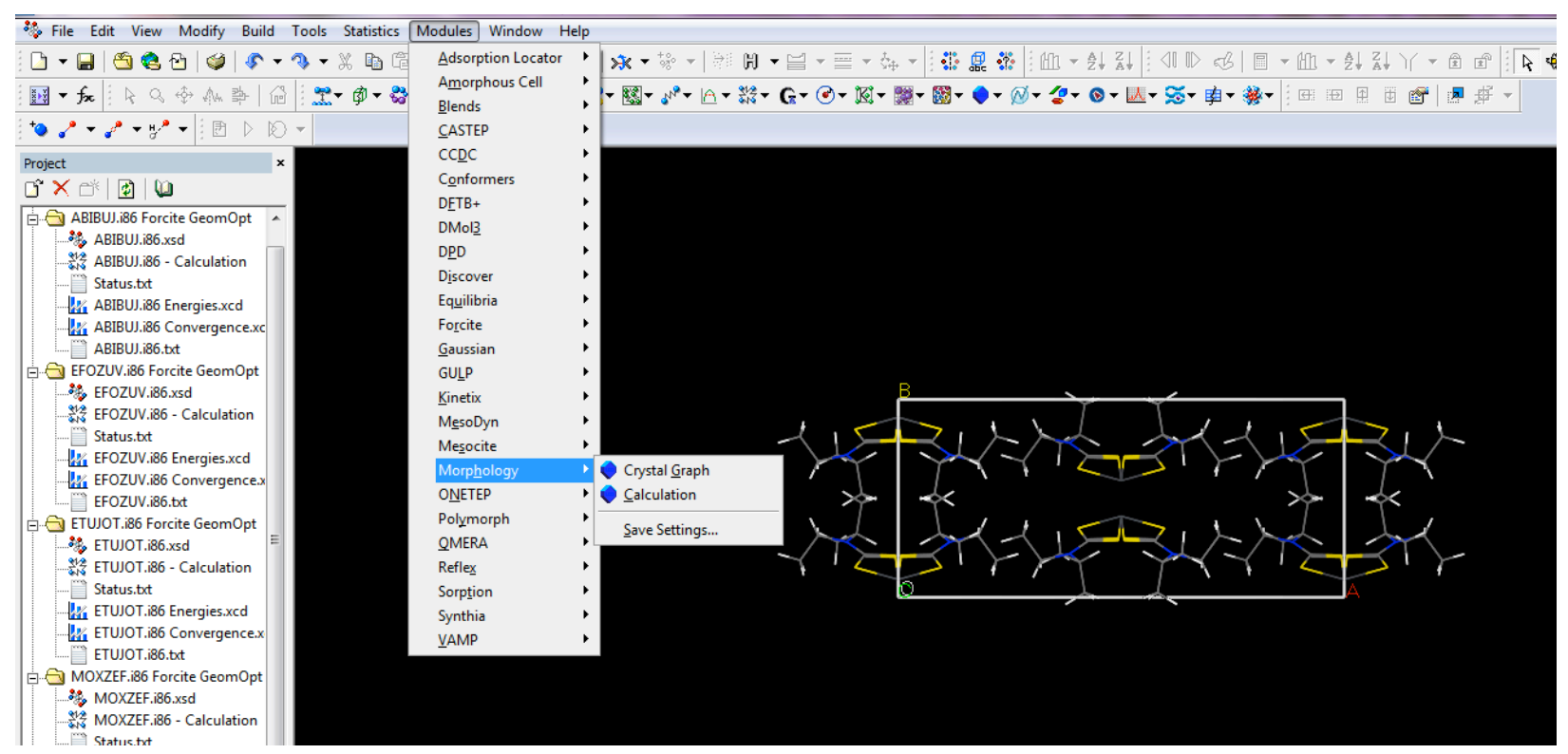


11. Using the crystal graph, the morphology was predicted via the Growth Morphology method. The energy method used for this step was Forcite and the quality was selected in the next step.

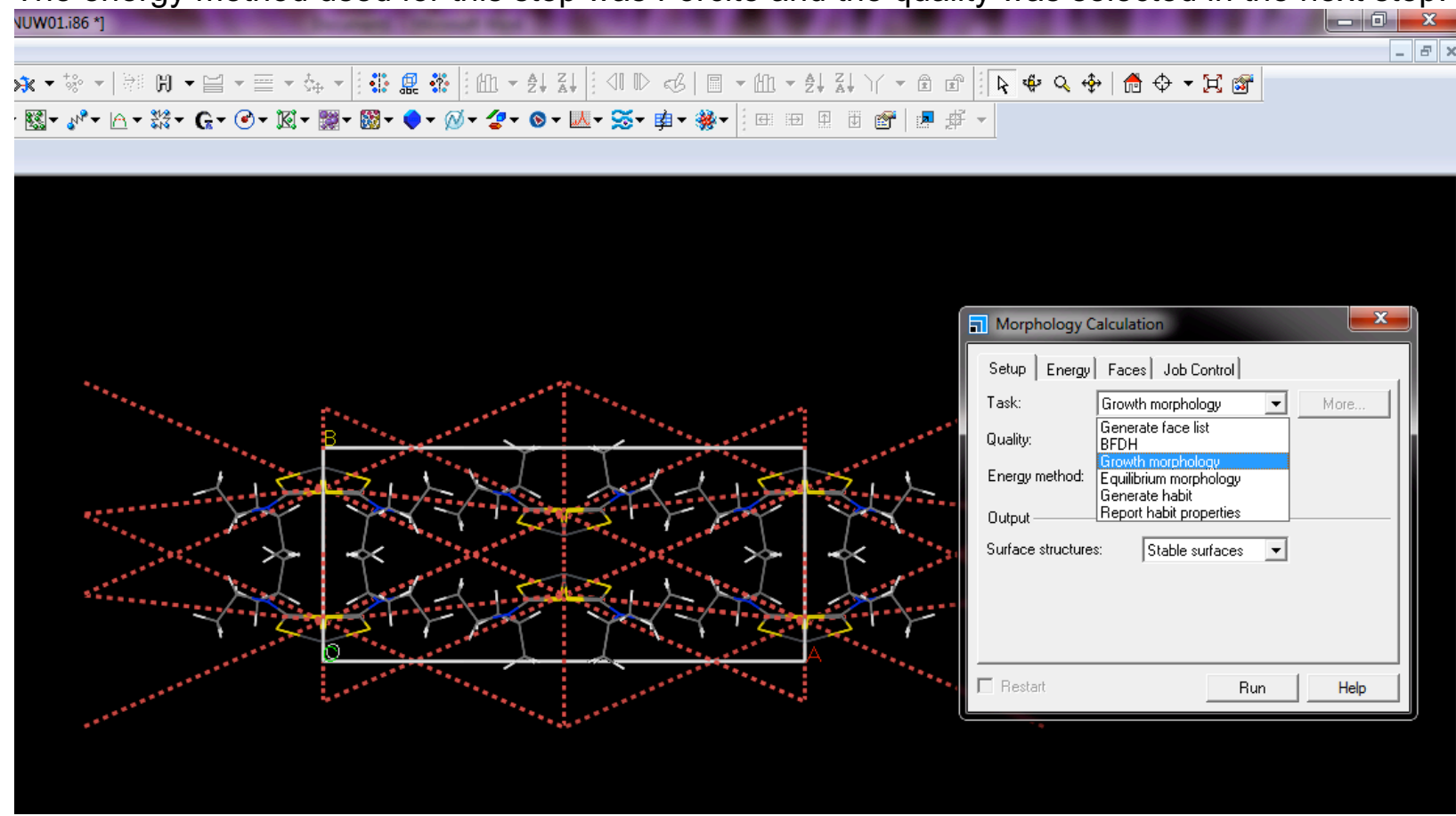

12. The calculation was performed using the UFF, charges assigned by Qeq, and an ultra-fine quality method. The summation method for electrostatic (Ewald) and van der Waals interactions (Atom-based) were default to the program.

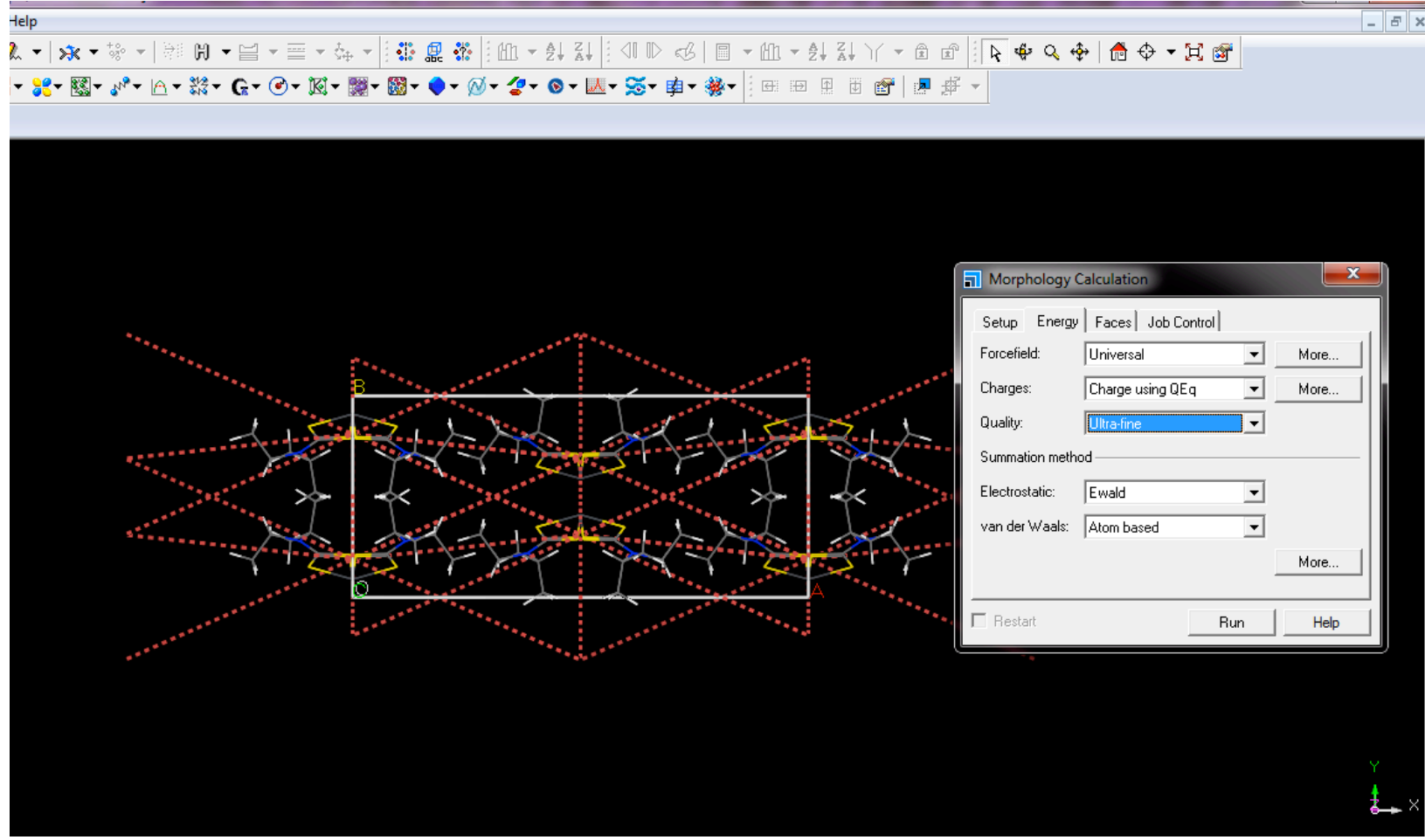


13. The morphology was then displayed and overlaid on the unit cell with the generated crystal graph.

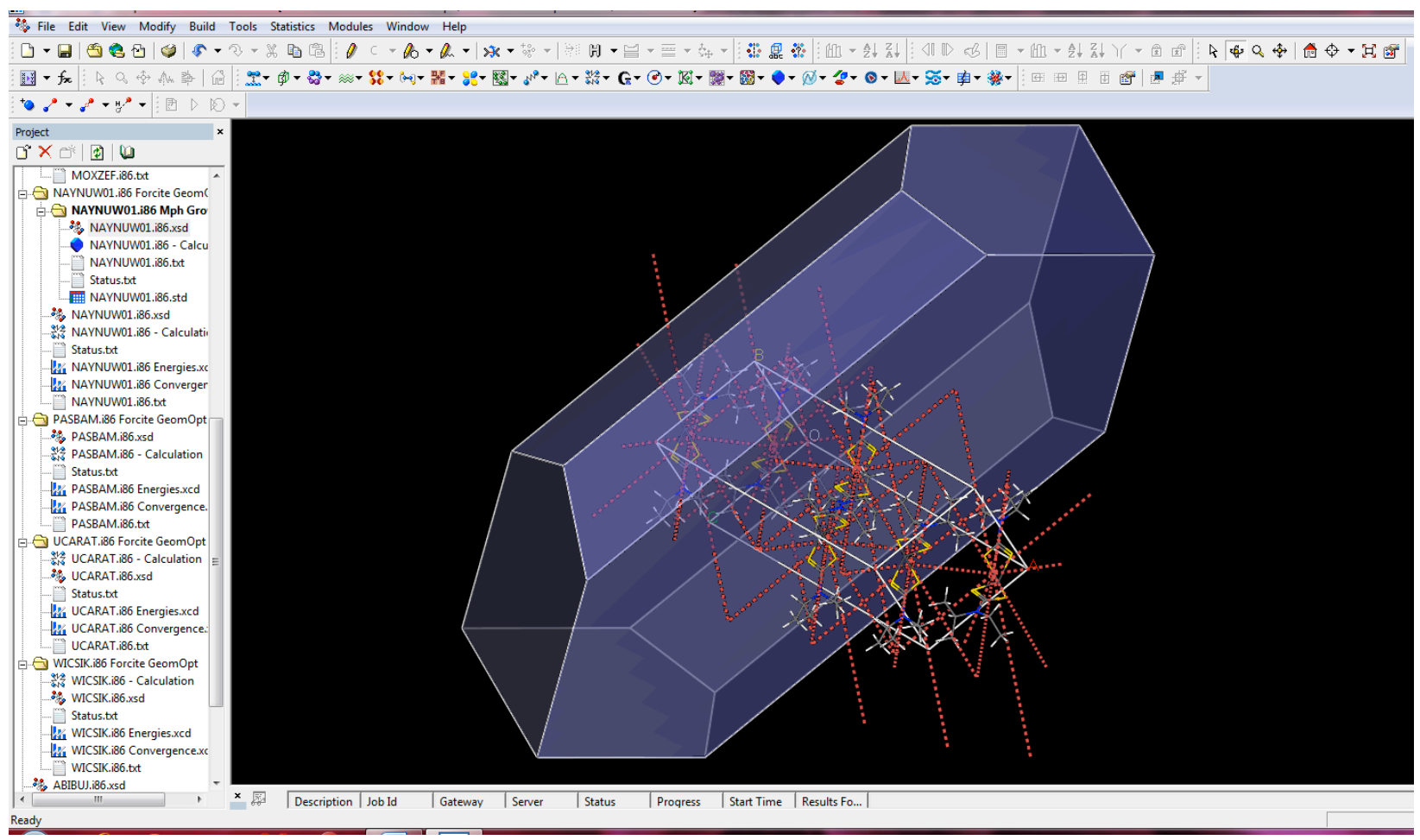

14. Details such as lattice energy and aspect ratio are listed in the .txt output file.

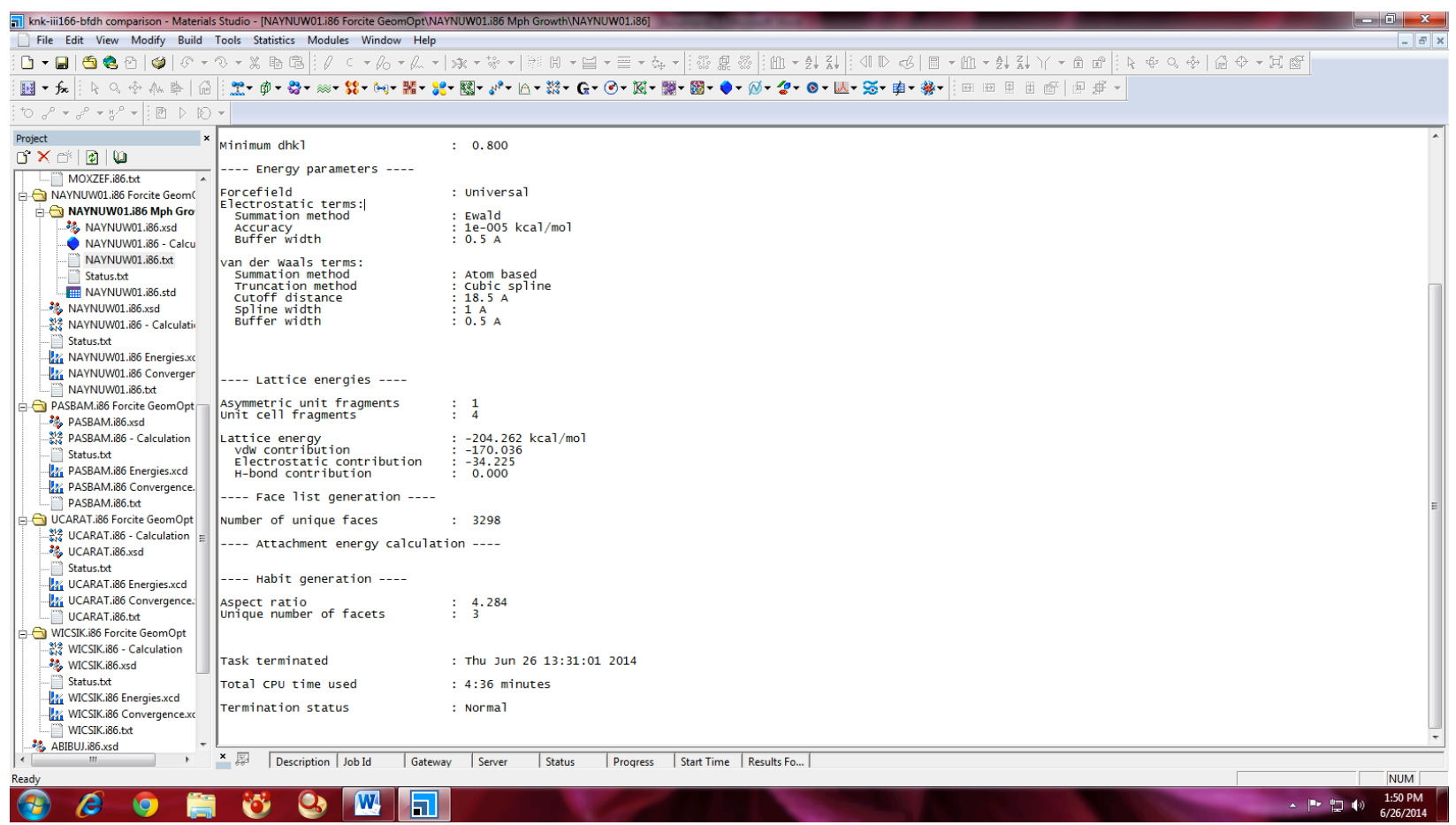




\section{Geometry Optimization}

Table S1. Unit cell parameters before and after unconstrained Forcite calculations.

\begin{tabular}{|c|c|c|c|c|c|c|c|c|c|c|c|c|c|c|c|c|c|c|}
\hline \multirow[b]{2}{*}{ Compound } & \multicolumn{6}{|c|}{ unoptimized lattice parameters } & \multicolumn{6}{|c|}{ optimized lattice parameters } & \multicolumn{6}{|c|}{$\%$ change } \\
\hline & $a(A)$ & b (A) & $c(A)$ & $a\left(^{\circ}\right)$ & $\beta\left(0^{\circ}\right)$ & $Y\left(^{\circ}\right)$ & $a(A)$ & b (A) & $c(\mathbf{A})$ & $\alpha\left(^{\circ}\right)$ & $\beta\left(0^{\circ}\right)$ & $Y\left(0^{\circ}\right)$ & $a(A)$ & b (A) & $c(\mathbf{A})$ & $\alpha\left(^{\circ}\right)$ & $\beta\left(0^{\circ}\right)$ & $Y\left(0^{\circ}\right)$ \\
\hline ABIBUJ & 10.614 & 12.278 & 13.462 & 95.311 & 111.434 & 104.811 & 14.755 & 12.753 & 9.664 & 86.330 & 112.110 & 109.503 & 39.015 & 3.869 & -28.213 & -9.423 & 0.607 & 4.477 \\
\hline ABUTEX01 & 10.079 & 10.779 & 11.928 & 74.665 & 70.677 & 85.778 & 13.405 & 10.514 & 9.819 & 55.656 & 82.432 & 90.714 & 32.994 & -2.458 & -17.684 & -25.459 & 16.632 & 5.754 \\
\hline ACAHUI & 6.543 & 13.575 & 16.172 & 90.000 & 97.561 & 90.000 & 7.505 & 12.226 & 16.783 & 90.000 & 112.352 & 90.000 & 14.704 & -9.935 & 3.779 & 0.000 & 15.161 & 0.000 \\
\hline ACASIH & 19.397 & 4.131 & 16.584 & 90.000 & 91.865 & 90.000 & 18.207 & 4.621 & 15.822 & 90.000 & 82.710 & 90.000 & -6.135 & 11.862 & -4.595 & 0.000 & -9.966 & 0.000 \\
\hline AJIMUB & 9.666 & 18.888 & 18.297 & 90.000 & 102.630 & 90.000 & 9.706 & 19.290 & 17.514 & 90.000 & 105.996 & 90.000 & 0.414 & 2.128 & -4.279 & 0.000 & 3.280 & 0.000 \\
\hline AJOGOW & 8.279 & 33.881 & 22.794 & 90.000 & 99.642 & 90.000 & 8.258 & 33.986 & 21.982 & 90.000 & 104.680 & 90.000 & -0.251 & 0.310 & -3.562 & 0.000 & 5.056 & 0.000 \\
\hline AKOHOY & 9.074 & 9.499 & 13.853 & 85.640 & 76.127 & 74.730 & 7.939 & 10.269 & 14.151 & 93.193 & 81.122 & 82.133 & -12.508 & 8.106 & 2.151 & 8.819 & 6.561 & 9.906 \\
\hline ATACUT & 8.672 & 13.196 & 28.629 & 90.000 & 93.538 & 90.000 & 8.671 & 12.703 & 27.400 & 90.000 & 97.705 & 90.000 & -0.006 & -3.733 & -4.293 & 0.000 & 4.455 & 0.000 \\
\hline AYUDON & 7.299 & 13.083 & 23.587 & 90.000 & 94.617 & 90.000 & 7.694 & 12.782 & 22.337 & 90.000 & 75.850 & 90.000 & 5.415 & -2.298 & -5.301 & 0.000 & -19.835 & 0.000 \\
\hline BAHKEC & 8.674 & 9.552 & 14.297 & 79.701 & 82.447 & 75.025 & 8.890 & 9.544 & 14.575 & 81.452 & 71.569 & 69.444 & 2.491 & -0.086 & 1.947 & 2.198 & -13.194 & -7.438 \\
\hline BAVDAE & 9.687 & 10.422 & 11.721 & 90.000 & 102.570 & 90.000 & 10.072 & 10.132 & 11.471 & 90.000 & 99.825 & 90.000 & 3.974 & -2.783 & -2.133 & 0.000 & -2.676 & 0.000 \\
\hline BEQWUQ & 14.973 & 9.902 & 20.581 & 90.000 & 109.379 & 90.000 & 14.293 & 10.536 & 20.433 & 90.000 & 107.860 & 90.000 & -4.542 & 6.398 & -0.719 & 0.000 & -1.389 & 0.000 \\
\hline BOJXOO & 8.797 & 10.005 & 11.993 & 66.280 & 74.318 & 68.154 & 8.590 & 9.910 & 12.017 & 69.280 & 75.979 & 68.602 & -2.358 & -0.946 & 0.199 & 4.526 & 2.235 & 0.657 \\
\hline BOJXUU & 12.987 & 14.107 & 14.792 & 90.000 & 95.445 & 90.000 & 13.603 & 13.032 & 15.253 & 90.000 & 95.876 & 90.000 & 4.747 & -7.622 & 3.120 & 0.000 & 0.452 & 0.000 \\
\hline BOSDOD & 10.136 & 11.166 & 11.314 & 95.460 & 116.336 & 111.668 & 10.327 & 14.137 & 11.542 & 89.958 & 120.998 & 126.543 & 1.884 & 26.608 & 2.012 & -5.764 & 4.007 & 13.321 \\
\hline BUMMOL & 11.600 & 8.975 & 6.863 & 97.790 & 90.190 & 101.540 & 11.054 & 9.193 & 7.069 & 111.566 & 81.278 & 98.243 & -4.707 & 2.429 & 3.002 & 14.087 & -9.881 & -3.247 \\
\hline BURPIO & 31.345 & 7.561 & 22.366 & 90.000 & 132.843 & 90.000 & 28.264 & 8.837 & 21.242 & 90.000 & 136.321 & 90.000 & -9.829 & 16.882 & -5.025 & 0.000 & 2.618 & 0.000 \\
\hline CEVLEV & 7.272 & 12.539 & 16.295 & 90.000 & 97.800 & 90.000 & 7.246 & 12.866 & 16.216 & 90.000 & 105.010 & 90.000 & -0.355 & 2.608 & -0.485 & 0.000 & 7.372 & 0.000 \\
\hline CIJQES & 10.407 & 12.034 & 12.470 & 89.464 & 72.864 & 70.868 & 10.239 & 12.206 & 12.465 & 83.572 & 73.182 & 66.897 & -1.614 & 1.427 & -0.040 & -6.586 & 0.436 & -5.603 \\
\hline CIJQIW & 10.414 & 12.084 & 12.468 & 89.224 & 72.815 & 70.638 & 10.275 & 12.242 & 12.490 & 83.380 & 72.863 & 66.682 & -1.332 & 1.308 & 0.179 & -6.550 & 0.066 & -5.600 \\
\hline CIJQIW01 & 10.361 & 12.033 & 12.431 & 89.330 & 72.910 & 70.560 & 10.267 & 12.341 & 12.495 & 83.032 & 72.925 & 66.064 & -0.907 & 2.560 & 0.515 & -7.050 & 0.021 & -6.372 \\
\hline CIJQOC & 22.366 & 11.214 & 11.602 & 90.000 & 101.411 & 90.000 & 21.980 & 11.371 & 11.556 & 90.000 & 102.964 & 90.000 & -1.724 & 1.396 & -0.397 & 0.000 & 1.531 & 0.000 \\
\hline CIQGUE & 8.241 & 23.765 & 8.341 & 90.000 & 112.910 & 90.000 & 8.056 & 24.544 & 8.176 & 90.000 & 111.938 & 90.000 & -2.245 & 3.278 & -1.978 & 0.000 & -0.861 & 0.000 \\
\hline
\end{tabular}




\begin{tabular}{|c|c|c|c|c|c|c|c|c|c|c|c|c|c|c|c|c|c|c|}
\hline \multirow[b]{2}{*}{ Compound } & \multicolumn{6}{|c|}{ unoptimized lattice parameters } & \multicolumn{6}{|c|}{ optimized lattice parameters } & \multicolumn{6}{|c|}{$\%$ change } \\
\hline & $\mathrm{a}(\mathrm{A})$ & $\mathrm{b}(\AA)$ & $c(\AA)$ & $\alpha\left(^{\circ}\right)$ & $\beta\left(^{\circ}\right)$ & $Y\left(^{\circ}\right)$ & $\mathrm{a}(\AA)$ & $\mathrm{b}(\mathrm{A})$ & $c(\AA)$ & $\alpha\left(^{\circ}\right)$ & $\beta\left(^{\circ}\right)$ & $Y\left(^{\circ}\right)$ & $\mathrm{a}(\AA)$ & $\mathrm{b}(\AA)$ & $c(\AA)$ & $\alpha\left(^{\circ}\right)$ & $\beta\left(^{\circ}\right)$ & $Y\left(^{\circ}\right)$ \\
\hline DAMTIV & 8.624 & 10.424 & 11.295 & 97.154 & 106.891 & 109.874 & 13.820 & 11.540 & 12.146 & 68.609 & 139.885 & 130.893 & 60.250 & 10.706 & 7.534 & -29.381 & 30.867 & 19.130 \\
\hline DAXMAR & 18.651 & 3.313 & 24.434 & 90.000 & 102.340 & 90.000 & 18.902 & 7.634 & 20.629 & 90.000 & 88.591 & 90.000 & 1.346 & 130.405 & -15.573 & 0.000 & -13.435 & 0.000 \\
\hline DOBZID10 & 14.831 & 16.722 & 8.399 & 90.000 & 95.270 & 90.000 & 25.979 & 10.844 & 15.666 & 90.000 & 151.688 & 90.000 & 75.167 & -35.151 & 86.522 & 0.000 & 59.219 & 0.000 \\
\hline DODMUF & 28.939 & 28.939 & 14.158 & 90.000 & 90.000 & 90.000 & 31.593 & 31.593 & 13.252 & 90.000 & 90.000 & 90.000 & 9.170 & 9.170 & -6.400 & 0.000 & 0.000 & 0.000 \\
\hline DUPXAO & 9.347 & 10.643 & 11.378 & 106.696 & 95.343 & 99.117 & 8.315 & 10.856 & 12.129 & 98.463 & 88.802 & 106.432 & -11.041 & 1.998 & 6.604 & -7.716 & -6.860 & 7.380 \\
\hline DURGON & 16.451 & 7.290 & 14.188 & 90.000 & 108.981 & 90.000 & 14.939 & 8.455 & 14.739 & 90.000 & 110.241 & 90.000 & -9.191 & 15.989 & 3.886 & 0.000 & 1.156 & 0.000 \\
\hline DURJEG & 7.320 & 8.307 & 23.325 & 90.000 & 90.397 & 90.000 & 9.359 & 8.364 & 19.843 & 90.000 & 97.218 & 90.000 & 27.860 & 0.692 & -14.927 & 0.000 & 7.546 & 0.000 \\
\hline EFOZUV & 10.266 & 12.304 & 14.844 & 92.054 & 103.473 & 105.561 & 9.545 & 13.479 & 14.831 & 105.421 & 107.958 & 101.508 & -7.025 & 9.552 & -0.090 & 14.521 & 4.334 & -3.839 \\
\hline EFUBAJ & 9.346 & 26.331 & 28.535 & 90.000 & 96.436 & 90.00 & 10.641 & 32.061 & 22.332 & 90.000 & 99.073 & 90.00 & 13.856 & 21.761 & -21.738 & 0.000 & 2.734 & 0.000 \\
\hline ERIGES & 9.011 & 10.923 & 11.920 & 83.760 & 87.626 & 71.601 & 10.756 & 12.175 & 10.616 & 90.177 & 99.483 & 54.424 & 19.365 & 11.462 & -10.940 & 7.661 & 13.531 & -23.990 \\
\hline ETUJOT & 21.937 & 7.457 & 23.238 & 90.000 & 90.000 & 90.000 & 20.955 & 7.285 & 23.188 & 90.000 & 90.000 & 90.000 & -4.476 & -2.305 & -0.215 & 0.000 & 0.000 & 0.000 \\
\hline FAZFIX & 26.226 & 4.585 & 15.541 & 90.000 & 119.667 & 90.000 & 24.927 & 4.243 & 15.344 & 90.000 & 93.605 & 90.000 & -4.952 & -7.457 & -1.268 & 0.000 & -21.779 & 0.000 \\
\hline FIHCUV & 10.267 & 22.017 & 7.451 & 90.000 & 92.432 & 90.000 & 9.765 & 27.507 & 7.264 & 90.000 & 98.725 & 90.000 & -4.889 & 24.935 & -2.515 & 0.000 & 6.808 & 0.000 \\
\hline FIMDUB & 12.859 & 13.074 & 17.064 & 90.000 & 105.504 & 90.000 & 13.281 & 12.320 & 18.866 & 90.000 & 113.607 & 90.000 & 3.280 & -5.764 & 10.560 & 0.000 & 7.680 & 0.000 \\
\hline FOFCEI & 10.569 & 11.082 & 17.918 & 72.784 & 88.671 & 85.959 & 10.459 & 10.911 & 18.158 & 72.294 & 90.740 & 85.128 & -1.039 & -1.543 & 1.339 & -0.673 & 2.333 & -0.967 \\
\hline FOHFEO & 35.937 & 8.337 & 25.432 & 90.000 & 126.076 & 90.000 & 38.377 & 8.618 & 27.536 & 90.000 & 138.411 & 90.000 & 6.790 & 3.371 & 8.273 & 0.000 & 9.784 & 0.000 \\
\hline FOPJIE & 10.240 & 13.111 & 17.619 & 90.000 & 98.972 & 90.000 & 9.831 & 13.140 & 18.749 & 90.000 & 104.587 & 90.000 & -3.995 & 0.219 & 6.412 & 0.000 & 5.673 & 0.000 \\
\hline GAGCIC & 17.201 & 14.170 & 19.873 & 90.000 & 115.644 & 90.000 & 16.686 & 13.075 & 21.344 & 90.000 & 121.852 & 90.000 & -2.995 & -7.724 & 7.404 & 0.000 & 5.368 & 0.000 \\
\hline GAGXES & 11.000 & 18.299 & 12.894 & 90.000 & 107.661 & 90.000 & 11.407 & 23.080 & 11.898 & 90.000 & 125.896 & 90.000 & 3.696 & 26.128 & -7.722 & 0.000 & 16.937 & 0.000 \\
\hline GICKUZ & 13.923 & 13.135 & 12.171 & 90.000 & 110.384 & 90.000 & 11.898 & 15.030 & 12.738 & 90.000 & 117.960 & 90.000 & -14.544 & 14.427 & 4.659 & 0.000 & 6.863 & 0.000 \\
\hline GIMPUN & 12.254 & 10.833 & 16.792 & 90.000 & 98.471 & 90.000 & 12.102 & 10.146 & 18.506 & 90.000 & 110.793 & 90.000 & -1.240 & -6.343 & 10.207 & 0.000 & 12.513 & 0.000 \\
\hline GODWIG & 10.057 & 11.074 & 14.553 & 69.707 & 73.897 & 74.244 & 9.966 & 10.990 & 14.826 & 68.456 & 70.264 & 73.177 & -0.903 & -0.762 & 1.879 & -1.795 & -4.916 & -1.437 \\
\hline GOJGUI & 9.217 & 11.587 & 19.103 & 102.516 & 97.867 & 97.715 & 11.648 & 11.572 & 19.079 & 76.687 & 111.152 & 91.375 & 26.377 & -0.129 & -0.127 & -25.195 & 13.575 & -6.488 \\
\hline GOLMEA & 8.258 & 8.805 & 9.677 & 103.976 & 98.262 & 108.190 & 8.178 & 13.321 & 9.390 & 121.433 & 62.028 & 129.831 & -0.968 & 51.286 & -2.961 & 16.789 & -36.875 & 20.003 \\
\hline GOLMIE & 13.742 & 18.068 & 18.611 & 90.000 & 90.000 & 90.000 & 14.058 & 14.732 & 22.969 & 90.000 & 90.000 & 90.000 & 2.299 & -18.464 & 23.414 & 0.000 & 0.000 & 0.000 \\
\hline GOLMOK & 5.305 & 11.820 & 17.493 & 90.000 & 94.569 & 90.000 & 4.797 & 12.300 & 23.090 & 90.000 & 122.005 & 90.000 & -9.574 & 4.061 & 31.997 & 0.000 & 29.012 & 0.000 \\
\hline GOPYIT & 18.920 & 9.647 & 22.275 & 90.000 & 112.592 & 90.000 & 24.055 & 10.084 & 24.978 & 90.000 & 139.233 & 90.000 & 27.141 & 4.526 & 12.135 & 0.000 & 23.662 & 0.000 \\
\hline GUWCOR & 11.234 & 11.946 & 13.513 & 100.361 & 109.481 & 100.996 & 11.234 & 11.946 & 13.513 & 100.361 & 109.481 & 100.996 & -0.002 & 0.001 & -0.002 & 0.000 & 0.000 & 0.000 \\
\hline
\end{tabular}




\begin{tabular}{|c|c|c|c|c|c|c|c|c|c|c|c|c|c|c|c|c|c|c|}
\hline \multirow[b]{2}{*}{ Compound } & \multicolumn{6}{|c|}{ unoptimized lattice parameters } & \multicolumn{6}{|c|}{ optimized lattice parameters } & \multicolumn{6}{|c|}{$\%$ change } \\
\hline & $\mathrm{a}(\AA)$ & $b(\AA)$ & $c(\AA)$ & $\alpha\left(^{\circ}\right)$ & $\beta\left(^{\circ}\right)$ & $Y\left(0^{\circ}\right)$ & $a(\AA)$ & $\mathrm{b}(\AA)$ & $c(\AA)$ & $a\left(^{\circ}\right)$ & $\beta\left(^{\circ}\right)$ & $Y\left(0^{\circ}\right)$ & $a(\AA)$ & $\mathrm{b}(\AA)$ & $c(\AA)$ & $\alpha\left(^{\circ}\right)$ & $\beta\left(^{\circ}\right)$ & $Y\left(0^{\circ}\right)$ \\
\hline HABGAU & 10.160 & 5.160 & 18.758 & 90.000 & 94.687 & 90.000 & 10.604 & 5.330 & 16.926 & 90.000 & 87.761 & 90.000 & 4.370 & 3.295 & -9.766 & 0.000 & -7.315 & 0.000 \\
\hline HAGSOX & 8.198 & 14.220 & 17.793 & 90.000 & 94.010 & 90.000 & 8.691 & 12.104 & 21.304 & 90.000 & 95.104 & 90.000 & 6.014 & -14.880 & 19.732 & 0.000 & 1.164 & 0.000 \\
\hline HEDNIO & 11.449 & 16.113 & 21.369 & 90.000 & 100.327 & 90.000 & 11.247 & 16.934 & 19.934 & 90.000 & 96.093 & 90.000 & -1.762 & 5.098 & -6.714 & 0.000 & -4.220 & 0.000 \\
\hline HEFHAB & 9.017 & 10.839 & 12.808 & 99.480 & 90.130 & 97.910 & 9.658 & 10.392 & 12.512 & 96.782 & 92.116 & 98.177 & 7.109 & -4.124 & -2.311 & -2.712 & 2.203 & 0.273 \\
\hline HERHUH & 11.226 & 9.820 & 12.258 & 90.000 & 100.500 & 90.000 & 11.601 & 8.498 & 12.636 & 90.000 & 96.041 & 90.000 & 3.340 & -13.462 & 3.084 & 0.000 & -4.437 & 0.000 \\
\hline HIQHEV & 11.054 & 20.906 & 11.291 & 90.000 & 101.667 & 90.000 & 11.197 & 18.383 & 12.201 & 90.000 & 102.365 & 90.000 & 1.295 & -12.068 & 8.064 & 0.000 & 0.687 & 0.000 \\
\hline HOXQOB & 15.841 & 16.146 & 40.504 & 90.000 & 90.000 & 90.000 & 15.996 & 17.016 & 40.739 & 90.000 & 90.000 & 90.000 & 0.981 & 5.387 & 0.580 & 0.000 & 0.000 & 0.000 \\
\hline HUGVUB & 10.439 & 11.973 & 12.424 & 90.450 & 107.020 & 108.520 & 10.655 & 11.532 & 13.064 & 78.686 & 111.469 & 104.704 & 2.069 & -3.683 & 5.151 & -13.006 & 4.157 & -3.516 \\
\hline HUGWAI & 10.146 & 31.930 & 19.622 & 90.000 & 99.340 & 90.000 & 10.293 & 31.688 & 19.524 & 90.000 & 100.663 & 90.000 & 1.449 & -0.758 & -0.499 & 0.000 & 1.332 & 0.000 \\
\hline HUGWEM & 8.760 & 11.529 & 16.702 & 75.200 & 82.280 & 87.860 & 8.501 & 11.509 & 17.478 & 75.095 & 80.794 & 87.639 & -2.959 & -0.173 & 4.646 & -0.140 & -1.806 & -0.252 \\
\hline IHEMUE & 19.532 & 11.008 & 7.219 & 90.000 & 112.325 & 90.000 & 18.689 & 12.016 & 7.699 & 90.000 & 119.122 & 90.000 & -4.318 & 9.154 & 6.649 & 0.000 & 6.051 & 0.000 \\
\hline IJONEB & 7.342 & 8.400 & 23.801 & 90.000 & 93.733 & 90.000 & 8.199 & 7.968 & 21.976 & 90.000 & 89.925 & 90.000 & 11.674 & -5.147 & -7.667 & 0.000 & -4.063 & 0.000 \\
\hline ILOBUH & 8.278 & 15.412 & 11.702 & 90.000 & 115.707 & 90.000 & 10.465 & 14.734 & 11.562 & 90.000 & 124.242 & 90.000 & 26.419 & -4.399 & -1.196 & 0.000 & 7.376 & 0.000 \\
\hline IVEFIY & 10.368 & 20.577 & 12.239 & 90.000 & 107.970 & 90.000 & 11.888 & 19.776 & 10.519 & 90.000 & 97.752 & 90.000 & 14.660 & -3.893 & -14.053 & 0.000 & -9.464 & 0.000 \\
\hline IWOVAR & 8.646 & 10.579 & 10.793 & 91.477 & 111.107 & 109.929 & 10.802 & 11.302 & 12.030 & 83.161 & 136.210 & 114.090 & 24.936 & 6.834 & 11.457 & -9.091 & 22.594 & 3.785 \\
\hline JADJIH & 9.576 & 14.151 & 16.175 & 104.840 & 102.260 & 90.620 & 8.714 & 13.934 & 18.230 & 116.432 & 94.878 & 85.338 & -9.002 & -1.533 & 12.705 & 11.057 & -7.219 & -5.829 \\
\hline JAWHAR & 27.659 & 16.275 & 17.166 & 90.000 & 121.620 & 90.000 & 32.441 & 15.032 & 16.468 & 90.000 & 127.242 & 90.000 & 17.289 & -7.637 & -4.066 & 0.000 & 4.623 & 0.000 \\
\hline JIBZAV & 12.107 & 11.738 & 15.540 & 90.000 & 111.090 & 90.000 & 11.478 & 11.929 & 14.897 & 90.000 & 108.707 & 90.000 & -5.195 & 1.627 & -4.138 & 0.000 & -2.145 & 0.000 \\
\hline JITBEU & 8.708 & 10.570 & 10.963 & 78.131 & 79.035 & 85.183 & 8.610 & 10.197 & 11.668 & 79.512 & 86.064 & 84.819 & -1.125 & -3.529 & 6.429 & 1.768 & 8.894 & -0.427 \\
\hline JUCCEP & 8.171 & 15.499 & 7.586 & 90.000 & 105.808 & 90.000 & 8.237 & 15.274 & 7.671 & 90.000 & 116.570 & 90.000 & 0.808 & -1.452 & 1.120 & 0.000 & 10.171 & 0.000 \\
\hline JUCCEP02 & 8.170 & 15.480 & 7.441 & 90.000 & 106.652 & 90.000 & 7.907 & 15.519 & 7.129 & 90.000 & 107.570 & 90.000 & -3.219 & 0.252 & -4.198 & 0.000 & 0.861 & 0.000 \\
\hline KAMKAL & 27.180 & 10.662 & 15.005 & 90.000 & 96.234 & 90.000 & 27.512 & 9.790 & 15.317 & 90.000 & 88.443 & 90.000 & 1.221 & -8.180 & 2.079 & 0.000 & -8.096 & 0.000 \\
\hline KEGZAX & 18.067 & 12.518 & 8.103 & 90.000 & 101.930 & 90.000 & 17.448 & 8.122 & 13.507 & 90.000 & 117.988 & 90.000 & -3.426 & -35.117 & 66.691 & 0.000 & 15.754 & 0.000 \\
\hline KEGZEB & 7.958 & 7.445 & 19.007 & 90.000 & 98.740 & 90.000 & 5.103 & 9.202 & 25.395 & 90.000 & 98.090 & 90.000 & -35.876 & 23.600 & 33.609 & 0.000 & -0.658 & 0.000 \\
\hline KILMEX & 11.837 & 11.993 & 14.611 & 90.000 & 97.930 & 90.000 & 11.473 & 12.779 & 13.441 & 90.000 & 99.405 & 90.000 & -3.075 & 6.554 & -8.008 & 0.000 & 1.506 & 0.000 \\
\hline KUJCIB & 8.382 & 9.648 & 11.427 & 102.792 & 105.623 & 90.303 & 7.903 & 8.960 & 12.127 & 101.967 & 105.329 & 83.500 & -5.719 & -7.128 & 6.130 & -0.803 & -0.278 & -7.534 \\
\hline $\mathrm{KUJCOH}$ & 8.126 & 12.294 & 10.685 & 90.000 & 110.170 & 90.000 & 9.059 & 14.138 & 8.469 & 90.000 & 124.764 & 90.000 & 11.482 & 14.999 & -20.739 & 0.000 & 13.247 & 0.000 \\
\hline KUQFAE & 7.856 & 8.416 & 9.155 & 78.524 & 69.771 & 82.813 & 7.181 & 8.738 & 9.488 & 81.426 & 70.125 & 89.107 & -8.588 & 3.832 & 3.637 & 3.696 & 0.507 & 7.600 \\
\hline
\end{tabular}




\begin{tabular}{|c|c|c|c|c|c|c|c|c|c|c|c|c|c|c|c|c|c|c|}
\hline \multirow[b]{2}{*}{ Compound } & \multicolumn{6}{|c|}{ unoptimized lattice parameters } & \multicolumn{6}{|c|}{ optimized lattice parameters } & \multicolumn{6}{|c|}{$\%$ change } \\
\hline & $\mathrm{a}(\AA)$ & $b(\AA)$ & $c(\AA)$ & $\alpha\left(^{\circ}\right)$ & $\beta\left(^{\circ}\right)$ & $Y\left(^{\circ}\right)$ & $a(\AA)$ & $\mathrm{b}(\AA)$ & $c(\AA)$ & $\alpha\left({ }^{\circ}\right)$ & $\beta\left(^{\circ}\right)$ & $Y\left(^{\circ}\right)$ & $a(\AA)$ & $\mathrm{b}(\AA)$ & $c(\AA)$ & $\alpha\left(^{\circ}\right)$ & $\beta\left(^{\circ}\right)$ & $Y\left(^{\circ}\right)$ \\
\hline KUQFAE01 & 12.037 & 5.731 & 16.327 & 90.000 & 90.368 & 90.000 & 10.881 & 5.931 & 18.889 & 90.000 & 76.913 & 90.000 & -9.604 & 3.490 & 15.692 & 0.000 & -14.889 & 0.000 \\
\hline LAJCAC & 8.436 & 10.391 & 12.075 & 90.000 & 103.421 & 90.000 & 8.001 & 10.795 & 11.009 & 90.000 & 96.622 & 90.000 & -5.156 & 3.891 & -8.831 & 0.000 & -6.574 & 0.000 \\
\hline LERQAB & 9.458 & 16.264 & 12.652 & 90.000 & 9.856 & 90.000 & 10.692 & 12.745 & 14.089 & 90.000 & 105.431 & 90.000 & 13.051 & -21.637 & 11.357 & 0.000 & 969.714 & 0.000 \\
\hline LEWPIM & 6.814 & 6.766 & 22.649 & 90.000 & 90.000 & 90.000 & 8.834 & 7.364 & 17.851 & 90.000 & 90.000 & 90.000 & 29.645 & 8.838 & -21.184 & 0.000 & 0.000 & 0.000 \\
\hline LIZFUW & 10.529 & 11.398 & 13.280 & 73.398 & 89.522 & 76.447 & 9.582 & 11.228 & 14.423 & 71.733 & 89.983 & 77.510 & -8.994 & -1.491 & 8.607 & -2.268 & 0.515 & 1.391 \\
\hline LIZGAD & 12.431 & 12.431 & 21.323 & 90.000 & 90.000 & 90.000 & 12.356 & 12.356 & 21.650 & 90.000 & 90.000 & 90.000 & -0.606 & -0.606 & 1.534 & 0.000 & 0.000 & 0.000 \\
\hline MAWNON & 14.646 & 15.501 & 15.429 & 90.000 & 99.583 & 90.000 & 12.660 & 17.708 & 16.606 & 90.000 & 105.783 & 90.000 & -13.560 & 14.238 & 7.628 & 0.000 & 6.226 & 0.000 \\
\hline MAWPAB & 16.212 & 14.420 & 25.146 & 90.000 & 92.014 & 90.000 & 16.005 & 13.903 & 25.521 & 90.000 & 89.892 & 90.000 & -1.277 & -3.584 & 1.491 & 0.000 & -2.306 & 0.000 \\
\hline MIFSIE02 & 17.709 & 13.410 & 12.902 & 90.000 & 104.896 & 90.000 & 18.157 & 14.166 & 12.028 & 90.000 & 113.026 & 90.000 & 2.531 & 5.641 & -6.777 & 0.000 & 7.751 & 0.000 \\
\hline MONLIK & 10.277 & 17.922 & 14.368 & 90.000 & 92.080 & 90.000 & 10.449 & 18.047 & 14.189 & 90.000 & 90.641 & 90.000 & 1.674 & 0.697 & -1.246 & 0.000 & -1.563 & 0.000 \\
\hline MOXYOO & 12.283 & 10.590 & 15.260 & 90.000 & 95.140 & 90.000 & 10.578 & 11.383 & 18.217 & 90.000 & 92.916 & 90.000 & -13.881 & 7.488 & 19.377 & 0.000 & -2.338 & 0.000 \\
\hline MOXZEF & 10.060 & 11.932 & 12.059 & 93.420 & 91.980 & 112.880 & 9.968 & 12.378 & 12.701 & 91.608 & 88.240 & 112.213 & -0.915 & 3.738 & 5.324 & -1.940 & -4.066 & -0.591 \\
\hline MTCBPB & 21.073 & 27.422 & 4.214 & 90.000 & 90.000 & 90.000 & 18.536 & 28.304 & 4.304 & 90.000 & 90.000 & 90.000 & -12.039 & 3.216 & 2.136 & 0.000 & 0.000 & 0.000 \\
\hline NADVEV & 9.710 & 11.816 & 17.999 & 72.985 & 88.178 & 89.040 & 10.125 & 11.552 & 17.841 & 71.669 & 84.076 & 85.525 & 4.279 & -2.233 & -0.875 & -1.803 & -4.652 & -3.948 \\
\hline NAKGIP & 21.078 & 42.546 & 8.388 & 90.000 & 90.000 & 90.000 & 21.074 & 43.174 & 8.283 & 90.000 & 90.000 & 90.000 & -0.019 & 1.476 & -1.252 & 0.000 & 0.000 & 0.000 \\
\hline NAPVOR & 18.117 & 10.216 & 8.654 & 90.000 & 112.000 & 90.000 & 14.469 & 8.172 & 13.598 & 90.000 & 111.780 & 90.000 & -20.136 & -20.009 & 57.137 & 0.000 & -0.196 & 0.000 \\
\hline NAYNUW01 & 22.478 & 9.718 & 8.734 & 90.000 & 102.620 & 90.000 & 22.011 & 9.179 & 8.770 & 90.000 & 106.838 & 90.000 & -2.078 & -5.546 & 0.412 & 0.000 & 4.110 & 0.000 \\
\hline NEDVAU & 10.100 & 11.287 & 11.615 & 77.868 & 89.884 & 84.785 & 9.576 & 11.148 & 12.041 & 74.788 & 96.502 & 88.741 & -5.191 & -1.233 & 3.670 & -3.955 & 7.363 & 4.666 \\
\hline NETVIR & 10.986 & 13.823 & 14.160 & 90.000 & 90.000 & 90.000 & 8.276 & 19.145 & 14.115 & 90.000 & 90.000 & 90.000 & -24.668 & 38.501 & -0.318 & 0.000 & 0.000 & 0.000 \\
\hline NIPFAU & 19.685 & 10.964 & 36.763 & 90.000 & 91.304 & 90.000 & 19.466 & 10.889 & 37.350 & 90.000 & 89.418 & 90.000 & -1.112 & -0.680 & 1.598 & 0.000 & -2.066 & 0.000 \\
\hline NIXTIX & 8.860 & 9.394 & 9.783 & 108.930 & 98.670 & 107.870 & 9.495 & 9.623 & 9.037 & 110.745 & 97.595 & 105.368 & 7.167 & 2.438 & -7.625 & 1.666 & -1.089 & -2.319 \\
\hline NOGQOQ & 8.314 & 19.379 & 21.059 & 90.000 & 90.000 & 90.000 & 8.327 & 19.687 & 20.814 & 90.000 & 90.000 & 90.000 & 0.156 & 1.588 & -1.164 & 0.000 & 0.000 & 0.000 \\
\hline NOMJUV & 11.763 & 11.763 & 11.918 & 90.000 & 90.000 & 120.000 & 10.028 & 10.028 & 13.426 & 90.000 & 90.000 & 120.000 & -14.753 & -14.753 & 12.653 & 0.000 & 0.000 & 0.000 \\
\hline NONXOE & 9.978 & 9.263 & 17.363 & 90.000 & 97.260 & 90.000 & 10.494 & 8.298 & 18.698 & 90.000 & 94.859 & 90.000 & 5.171 & -10.420 & 7.689 & 0.000 & -2.469 & 0.000 \\
\hline NUFQEL02 & 10.394 & 10.776 & 11.462 & 90.000 & 90.000 & 90.000 & 9.306 & 10.933 & 12.809 & 90.000 & 90.000 & 90.000 & -10.466 & 1.458 & 11.753 & 0.000 & 0.000 & 0.000 \\
\hline NUNXOK & 22.439 & 4.764 & 15.714 & 90.000 & 101.167 & 90.000 & 21.704 & 6.423 & 13.431 & 90.000 & 97.606 & 90.000 & -3.276 & 34.835 & -14.528 & 0.000 & -3.520 & 0.000 \\
\hline NUWHUJ & 11.608 & 11.746 & 12.603 & 107.979 & 112.338 & 100.396 & 11.476 & 12.905 & 12.872 & 103.118 & 114.593 & 113.477 & -1.140 & 9.864 & 2.135 & -4.502 & 2.007 & 13.029 \\
\hline
\end{tabular}




\begin{tabular}{|c|c|c|c|c|c|c|c|c|c|c|c|c|c|c|c|c|c|c|}
\hline \multirow[b]{2}{*}{ Compound } & \multicolumn{6}{|c|}{ unoptimized lattice parameters } & \multicolumn{6}{|c|}{ optimized lattice parameters } & \multicolumn{6}{|c|}{$\%$ change } \\
\hline & $\mathrm{a}(\AA)$ & $\mathrm{b}(\AA)$ & $c(\AA)$ & $\alpha\left(^{\circ}\right)$ & $\beta\left(^{\circ}\right)$ & $y\left(0^{\circ}\right)$ & $\mathrm{a}(\AA)$ & $b(\AA)$ & $c(\AA)$ & $\alpha\left(\left(^{\circ}\right)\right.$ & $\beta\left(0^{\circ}\right)$ & $Y\left(^{\circ}\right)$ & $\mathrm{a}(\AA)$ & $\mathrm{b}(\AA)$ & $c(\AA)$ & $\alpha\left(^{\circ}\right)$ & $\beta\left(^{\circ}\right)$ & $y\left(0^{\circ}\right)$ \\
\hline OJAMIW & 9.883 & 16.419 & 13.971 & 90.000 & 131.564 & 90.000 & 10.585 & 14.587 & 14.860 & 90.000 & 136.089 & 90.000 & 7.103 & -11.157 & 6.363 & 0.000 & 3.439 & 0.000 \\
\hline OKIMUR & 10.114 & 10.019 & 17.394 & 90.000 & 96.934 & 90.000 & 11.969 & 8.087 & 18.925 & 90.000 & 95.501 & 90.000 & 18.342 & -19.283 & 8.803 & 0.000 & -1.478 & 0.000 \\
\hline OSAKAV & 10.483 & 12.033 & 8.126 & 90.000 & 91.891 & 90.000 & 7.378 & 16.473 & 8.080 & 90.000 & 80.323 & 90.000 & -29.619 & 36.903 & -0.562 & 0.000 & -12.589 & 0.000 \\
\hline OSAKEZ & 13.196 & 12.445 & 15.242 & 90.000 & 109.177 & 90.000 & 13.829 & 11.224 & 14.934 & 90.000 & 110.511 & 90.000 & 4.796 & -9.811 & -2.020 & 0.000 & 1.222 & 0.000 \\
\hline OXURUV & 10.719 & 12.040 & 12.130 & 103.458 & 108.499 & 94.887 & 10.535 & 11.036 & 13.030 & 90.874 & 115.029 & 96.734 & -1.714 & -8.336 & 7.424 & -12.163 & 6.018 & 1.947 \\
\hline OZCDPB & 8.400 & 15.082 & 17.650 & 90.000 & 106.330 & 90.000 & 8.761 & 14.496 & 19.941 & 90.000 & 53.339 & 90.000 & 4.298 & -3.885 & 12.980 & 0.000 & -49.836 & 0.000 \\
\hline PAQVIN & 9.168 & 9.588 & 16.707 & 90.000 & 95.410 & 90.000 & 13.358 & 12.469 & 9.992 & 90.000 & 82.140 & 90.000 & 45.702 & 30.048 & -40.193 & 0.000 & -13.908 & 0.000 \\
\hline PASBAM & 7.180 & 10.346 & 17.712 & 90.000 & 90.000 & 90.000 & 7.873 & 13.363 & 14.308 & 90.000 & 90.000 & 90.000 & 9.647 & 29.161 & -19.219 & 0.000 & 0.000 & 0.000 \\
\hline PIGWAE & 12.331 & 12.587 & 15.119 & 105.315 & 100.911 & 108.965 & 12.579 & 12.552 & 14.902 & 107.919 & 99.756 & 106.588 & 2.010 & -0.276 & -1.432 & 2.473 & -1.145 & -2.181 \\
\hline PILYAK & 7.856 & 8.148 & 13.788 & 74.390 & 82.770 & 77.210 & 7.931 & 8.986 & 13.197 & 74.836 & 73.391 & 66.589 & 0.955 & 10.285 & -4.286 & 0.600 & -11.331 & -13.756 \\
\hline POGDOE & 10.523 & 8.992 & 16.672 & 90.000 & 104.142 & 90.000 & 10.677 & 8.164 & 18.618 & 90.000 & 106.920 & 90.000 & 1.463 & -9.210 & 11.672 & 0.000 & 2.668 & 0.000 \\
\hline PUHYAT & 9.551 & 10.081 & 13.248 & 109.865 & 97.322 & 90.643 & 11.149 & 9.832 & 12.939 & 118.252 & 86.881 & 90.337 & 16.731 & -2.465 & -2.335 & 7.634 & -10.728 & -0.338 \\
\hline PUQVON & 16.160 & 8.097 & 15.378 & 90.000 & 110.982 & 90.000 & 16.122 & 7.593 & 16.507 & 90.000 & 110.874 & 90.000 & -0.233 & -6.228 & 7.342 & 0.000 & -0.097 & 0.000 \\
\hline PUQVUT & 27.517 & 10.674 & 13.075 & 90.000 & 100.837 & 90.000 & 27.191 & 9.635 & 15.104 & 90.000 & 101.365 & 90.000 & -1.185 & -9.734 & 15.518 & 0.000 & 0.524 & 0.000 \\
\hline QAFPIY & 14.745 & 9.539 & 30.317 & 90.000 & 90.000 & 90.000 & 14.859 & 9.191 & 30.676 & 90.000 & 90.000 & 90.000 & 0.774 & -3.648 & 1.186 & 0.000 & 0.000 & 0.000 \\
\hline QEYSET & 9.662 & 25.527 & 11.181 & 90.000 & 90.383 & 90.000 & 9.382 & 27.934 & 10.649 & 90.000 & 100.158 & 90.000 & -2.898 & 9.429 & -4.758 & 0.000 & 10.815 & 0.000 \\
\hline QEYSIX & 10.437 & 11.251 & 12.736 & 115.124 & 95.840 & 92.309 & 9.535 & 11.392 & 12.661 & 106.736 & 95.640 & 81.777 & -8.641 & 1.253 & -0.589 & -7.286 & -0.209 & -11.410 \\
\hline QIBSIE & 18.820 & 9.397 & 16.324 & 90.000 & 109.390 & 90.000 & 17.843 & 10.758 & 15.487 & 90.000 & 105.564 & 90.000 & -5.191 & 14.483 & -5.127 & 0.000 & -3.498 & 0.000 \\
\hline QOKHAZ & 9.980 & 17.863 & 12.193 & 90.000 & 112.510 & 90.000 & 9.604 & 18.305 & 12.043 & 90.000 & 109.736 & 90.000 & -3.768 & 2.474 & -1.230 & 0.000 & -2.466 & 0.000 \\
\hline QOPJAH & 9.295 & 9.931 & 16.482 & 84.867 & 79.842 & 66.327 & 9.237 & 8.916 & 19.866 & 79.178 & 63.749 & 66.938 & -0.624 & -10.221 & 20.532 & -6.703 & -20.156 & 0.921 \\
\hline QUXQAC & 19.916 & 8.017 & 16.358 & 90.000 & 90.000 & 90.000 & 20.837 & 8.067 & 14.961 & 90.000 & 90.000 & 90.000 & 4.622 & 0.620 & -8.537 & 0.000 & 0.000 & 0.000 \\
\hline RABKEK & 9.332 & 10.025 & 11.668 & 68.410 & 68.390 & 68.580 & 7.824 & 9.122 & 12.240 & 77.150 & 76.150 & 69.550 & -16.159 & -9.007 & 4.902 & 12.776 & 11.347 & 1.414 \\
\hline RAQFAQ01 & 5.323 & 12.293 & 13.081 & 90.000 & 101.551 & 90.000 & 5.496 & 12.921 & 12.360 & 90.000 & 98.132 & 90.000 & 3.244 & 5.109 & -5.513 & 0.000 & -3.367 & 0.000 \\
\hline RAQQOP & 10.834 & 9.023 & 8.906 & 80.340 & 89.400 & 79.780 & 10.186 & 9.373 & 8.752 & 91.044 & 81.269 & 97.562 & -5.981 & 3.879 & -1.729 & 13.323 & -9.095 & 22.289 \\
\hline RASRAE & 14.224 & 8.882 & 22.885 & 90.000 & 101.470 & 90.000 & 14.169 & 10.257 & 19.136 & 90.000 & 98.348 & 90.000 & -0.387 & 15.481 & -16.382 & 0.000 & -3.077 & 0.000 \\
\hline SAHRID & 13.316 & 14.179 & 14.614 & 90.000 & 97.539 & 90.000 & 13.667 & 14.014 & 14.392 & 90.000 & 96.919 & 90.000 & 2.633 & -1.165 & -1.522 & 0.000 & -0.636 & 0.000 \\
\hline SAHSIE & 8.043 & 18.649 & 27.034 & 90.000 & 96.516 & 90.000 & 7.869 & 19.263 & 26.530 & 90.000 & 96.577 & 90.000 & -2.161 & 3.295 & -1.866 & 0.000 & 0.063 & 0.000 \\
\hline SIFKAU & 11.873 & 12.653 & 13.005 & 103.320 & 106.940 & 115.980 & 12.686 & 12.164 & 11.167 & 96.738 & 106.831 & 108.434 & 6.847 & -3.865 & -14.136 & -6.370 & -0.102 & -6.506 \\
\hline
\end{tabular}




\begin{tabular}{|c|c|c|c|c|c|c|c|c|c|c|c|c|c|c|c|c|c|c|}
\hline \multirow[b]{2}{*}{ Compound } & \multicolumn{6}{|c|}{ unoptimized lattice parameters } & \multicolumn{6}{|c|}{ optimized lattice parameters } & \multicolumn{6}{|c|}{$\%$ change } \\
\hline & $a(A)$ & $\mathrm{b}(\mathrm{A})$ & $c(\AA)$ & $\alpha\left(^{\circ}\right)$ & $\beta\left(^{\circ}\right)$ & $y\left(0^{\circ}\right)$ & $a(A)$ & $\mathrm{b}(\mathrm{A})$ & $c(\AA)$ & $\alpha\left(^{\circ}\right)$ & $\beta\left(^{\circ}\right)$ & $y\left(0^{\circ}\right)$ & $\mathrm{a}(\AA)$ & $\mathrm{b}(\AA)$ & $c(\AA)$ & $\alpha\left(^{\circ}\right)$ & $\beta\left(^{\circ}\right)$ & $Y\left(0^{\circ}\right)$ \\
\hline SUCNUZ & 10.625 & 11.864 & 15.086 & 77.860 & 68.210 & 69.510 & 10.660 & 12.823 & 14.738 & 73.258 & 57.854 & 68.233 & 0.329 & 8.083 & -2.307 & -5.911 & -15.183 & -1.837 \\
\hline SUXZOB & 10.341 & 10.833 & 11.418 & 89.923 & 72.080 & 65.273 & 10.601 & 10.532 & 12.661 & 97.937 & 58.733 & 90.451 & 2.512 & -2.776 & 10.888 & 8.912 & -18.517 & 38.573 \\
\hline TAMBUF & 9.431 & 10.950 & 11.609 & 80.900 & 84.410 & 73.360 & 9.481 & 11.202 & 11.995 & 85.529 & 92.192 & 62.185 & 0.527 & 2.301 & 3.325 & 5.722 & 9.219 & -15.233 \\
\hline TASCIZ & 11.032 & 9.187 & 25.426 & 90.000 & 96.860 & 90.000 & 9.638 & 9.933 & 26.255 & 90.000 & 106.742 & 90.000 & -12.636 & 8.120 & 3.260 & 0.000 & 10.202 & 0.000 \\
\hline TATREM & 20.640 & 7.454 & 22.897 & 90.000 & 101.164 & 90.000 & 18.397 & 8.805 & 20.353 & 90.000 & 95.258 & 90.000 & -10.866 & 18.118 & -11.109 & 0.000 & -5.838 & 0.000 \\
\hline TIWCUX & 21.086 & 10.831 & 15.899 & 90.000 & 118.930 & 90.000 & 20.251 & 11.236 & 15.948 & 90.000 & 118.656 & 90.000 & -3.960 & 3.739 & 0.308 & 0.000 & -0.230 & 0.000 \\
\hline TOJPIR & 11.237 & 10.992 & 8.139 & 90.000 & 97.320 & 90.000 & 8.062 & 13.022 & 7.658 & 90.000 & 76.439 & 90.000 & -28.255 & 18.468 & -5.910 & 0.000 & -21.456 & 0.000 \\
\hline TOXPEB & 9.252 & 9.742 & 11.141 & 75.920 & 77.040 & 87.340 & 9.970 & 9.803 & 10.632 & 82.121 & 61.905 & 87.535 & 7.760 & 0.626 & -4.569 & 8.168 & -19.646 & 0.223 \\
\hline TUMFAJ & 18.544 & 11.924 & 20.901 & 90.000 & 96.231 & 90.000 & 17.297 & 12.579 & 22.457 & 90.000 & 94.059 & 90.000 & -6.725 & 5.493 & 7.445 & 0.000 & -2.257 & 0.000 \\
\hline UCARAT & 9.593 & 10.899 & 11.246 & 99.450 & 109.678 & 96.891 & 9.816 & 15.701 & 10.213 & 104.902 & 88.892 & 130.473 & 2.321 & 44.066 & -9.188 & 5.482 & -18.952 & 34.660 \\
\hline UDIKOI & 7.792 & 11.332 & 13.888 & 66.876 & 82.502 & 73.039 & 9.396 & 13.510 & 11.980 & 67.895 & 60.576 & 70.771 & 20.584 & 19.219 & -13.738 & 1.524 & -26.576 & -3.105 \\
\hline ULAVUZ & 9.615 & 11.534 & 13.272 & 82.459 & 79.280 & 67.369 & 12.131 & 11.216 & 11.846 & 72.319 & 86.034 & 77.593 & 26.169 & -2.757 & -10.747 & -12.297 & 8.519 & 15.176 \\
\hline ULAWAG & 7.703 & 15.995 & 18.893 & 90.000 & 100.919 & 90.000 & 7.505 & 16.947 & 19.680 & 90.000 & 103.165 & 90.000 & -2.574 & 5.953 & 4.166 & 0.000 & 2.226 & 0.000 \\
\hline VAMWIP01 & 17.505 & 13.383 & 6.792 & 90.000 & 112.250 & 90.000 & 13.588 & 10.356 & 11.635 & 90.000 & 70.566 & 90.000 & -22.376 & -22.618 & 71.315 & 0.000 & -37.135 & 0.000 \\
\hline VEJNIH & 8.049 & 18.927 & 31.080 & 90.000 & 90.000 & 90.000 & 8.650 & 19.302 & 27.423 & 90.000 & 90.000 & 90.000 & 7.467 & 1.981 & -11.766 & 0.000 & 0.000 & 0.000 \\
\hline VEWREU & 14.231 & 10.487 & 15.814 & 84.950 & 144.280 & 91.890 & 14.235 & 10.329 & 15.839 & 85.499 & 145.660 & 92.182 & 0.028 & -1.507 & 0.158 & 0.646 & 0.956 & 0.318 \\
\hline VOCHIF & 11.047 & 14.486 & 32.048 & 91.300 & 99.730 & 101.610 & 10.978 & 14.534 & 32.153 & 89.413 & 103.914 & 96.859 & -0.625 & 0.331 & 0.328 & -2.067 & 4.195 & -4.676 \\
\hline WABQEX & 9.278 & 11.441 & 12.276 & 73.820 & 72.754 & 68.680 & 9.975 & 14.661 & 20.166 & 48.055 & 33.986 & 61.717 & 7.517 & 28.146 & 64.266 & -34.902 & -53.286 & -10.138 \\
\hline WACCUA & 10.909 & 4.827 & 15.980 & 90.000 & 100.020 & 90.000 & 11.570 & 4.626 & 16.347 & 90.000 & 92.869 & 90.000 & 6.059 & -4.166 & 2.297 & 0.000 & -7.150 & 0.000 \\
\hline WAJQON01 & 20.457 & 11.292 & 8.409 & 90.000 & 91.370 & 90.000 & 17.174 & 17.712 & 9.756 & 90.000 & 61.856 & 90.000 & -16.048 & 56.854 & 16.019 & 0.000 & -32.302 & 0.000 \\
\hline WALQIK & 8.737 & 16.261 & 6.951 & 90.000 & 94.824 & 90.000 & 8.055 & 14.478 & 8.326 & 90.000 & 89.383 & 90.000 & -7.806 & -10.965 & 19.781 & 0.000 & -5.738 & 0.000 \\
\hline WALQOQ & 9.027 & 17.016 & 7.210 & 90.000 & 95.360 & 90.000 & 8.105 & 18.698 & 7.273 & 90.000 & 100.052 & 90.000 & -10.214 & 9.885 & 0.874 & 0.000 & 4.920 & 0.000 \\
\hline WAQBIZ & 7.720 & 12.880 & 14.316 & 111.051 & 97.022 & 98.520 & 9.658 & 11.537 & 13.996 & 88.643 & 113.279 & 117.475 & 25.104 & -10.427 & -2.235 & -20.178 & 16.756 & 19.240 \\
\hline WATSEP & 10.180 & 25.275 & 9.665 & 90.000 & 90.000 & 90.000 & 7.396 & 12.944 & 14.244 & 111.125 & 103.005 & 98.951 & -27.347 & -48.787 & 47.379 & 23.472 & 14.450 & 9.946 \\
\hline WICSIK & 10.412 & 11.884 & 12.742 & 89.892 & 78.783 & 81.928 & 10.765 & 11.718 & 12.164 & 90.020 & 88.388 & 87.887 & 3.387 & -1.398 & -4.538 & 0.142 & 12.192 & 7.273 \\
\hline WILNOV & 8.770 & 9.733 & 12.857 & 81.219 & 81.435 & 74.993 & 10.963 & 7.413 & 13.924 & 101.376 & 75.270 & 81.233 & 25.006 & -23.836 & 8.299 & 24.817 & -7.570 & 8.321 \\
\hline WIMKIN & 14.196 & 10.984 & 15.590 & 90.000 & 116.482 & 90.000 & 13.468 & 12.130 & 14.590 & 90.000 & 113.596 & 90.000 & -5.126 & 10.432 & -6.416 & 0.000 & -2.478 & 0.000 \\
\hline WITQOF & 11.104 & 12.671 & 13.124 & 92.420 & 109.300 & 99.480 & 11.394 & 12.874 & 13.843 & 98.317 & 114.883 & 106.096 & 2.612 & 1.602 & 5.479 & 6.381 & 5.108 & 6.651 \\
\hline
\end{tabular}




\begin{tabular}{|c|c|c|c|c|c|c|c|c|c|c|c|c|c|c|c|c|c|c|}
\hline \multirow[b]{2}{*}{ Compound } & \multicolumn{6}{|c|}{ unoptimized lattice parameters } & \multicolumn{6}{|c|}{ optimized lattice parameters } & \multicolumn{6}{|c|}{$\%$ change } \\
\hline & $a(\AA)$ & $\mathrm{b}(\mathrm{A})$ & $c(\AA)$ & $\alpha\left({ }^{\circ}\right)$ & $\beta\left(^{\circ}\right)$ & $\mathrm{Y}\left({ }^{\circ}\right)$ & $a(\AA)$ & $\mathrm{b}(\AA)$ & $c(\AA)$ & $\alpha\left(^{\circ}\right)$ & $\beta\left(^{\circ}\right)$ & $y\left({ }^{\circ}\right)$ & $a(\AA)$ & $\mathrm{b}(\mathrm{A})$ & $c(\AA)$ & $\alpha\left(^{\circ}\right)$ & $\beta\left(^{\circ}\right)$ & $Y\left({ }^{\circ}\right)$ \\
\hline WOCYUJ & 15.300 & 23.967 & 20.180 & 90.000 & 99.930 & 90.000 & 14.797 & 24.220 & 20.072 & 90.000 & 97.840 & 90.000 & -3.288 & 1.056 & -0.535 & 0.000 & -2.091 & 0.000 \\
\hline WUXGAY & 15.755 & 17.460 & 20.784 & 90.000 & 115.010 & 90.000 & 17.444 & 17.996 & 18.955 & 90.000 & 80.248 & 90.000 & 10.720 & 3.069 & -8.800 & 0.000 & -30.225 & 0.000 \\
\hline XAGKEX & 7.413 & 10.923 & 12.087 & 98.073 & 106.450 & 99.980 & 8.421 & 12.828 & 10.785 & 99.764 & 111.874 & 100.541 & 13.605 & 17.440 & -10.770 & 1.724 & 5.095 & 0.561 \\
\hline XAGKIB & 7.209 & 12.928 & 16.536 & 90.000 & 115.624 & 90.000 & 7.374 & 12.712 & 17.593 & 90.000 & 121.178 & 90.000 & 2.283 & -1.671 & 6.392 & 0.000 & 4.804 & 0.000 \\
\hline XANCOG & 11.108 & 12.614 & 11.312 & 99.070 & 110.680 & 109.920 & 12.257 & 12.483 & 12.024 & 103.673 & 111.657 & 114.609 & 10.344 & -1.039 & 6.294 & 4.646 & 0.883 & 4.266 \\
\hline XIJFOL & 16.857 & 13.390 & 8.871 & 90.000 & 109.191 & 90.000 & 18.537 & 13.243 & 8.495 & 90.000 & 112.584 & 90.000 & 9.966 & -1.094 & -4.234 & 0.000 & 3.107 & 0.000 \\
\hline XIRBUW & 13.781 & 14.517 & 21.279 & 90.000 & 90.000 & 90.000 & 13.919 & 14.352 & 20.982 & 90.000 & 90.000 & 90.000 & 1.001 & -1.137 & -1.396 & 0.000 & 0.000 & 0.000 \\
\hline XIRCEH & 28.043 & 9.858 & 18.872 & 90.000 & 116.997 & 90.000 & 29.571 & 9.476 & 19.042 & 90.000 & 114.079 & 90.000 & 5.449 & -3.875 & 0.901 & 0.000 & -2.494 & 0.000 \\
\hline XISSAT & 9.295 & 13.680 & 15.390 & 90.000 & 98.365 & 90.000 & 10.019 & 12.763 & 15.193 & 90.000 & 86.553 & 90.000 & 7.793 & -6.700 & -1.282 & 0.000 & -12.008 & 0.000 \\
\hline XOPDUB & 8.158 & 9.070 & 11.019 & 68.089 & 82.769 & 74.970 & 8.521 & 8.554 & 12.816 & 58.733 & 79.806 & 71.508 & 4.448 & -5.686 & 16.311 & -13.741 & -3.580 & -4.618 \\
\hline XUZZAU & 8.698 & 12.120 & 15.115 & 91.885 & 98.639 & 97.444 & 8.485 & 11.937 & 15.280 & 90.983 & 95.889 & 95.286 & -2.448 & -1.506 & 1.094 & -0.982 & -2.788 & -2.215 \\
\hline YAGLAT01 & 9.943 & 12.229 & 12.616 & 112.530 & 82.570 & 91.550 & 8.965 & 11.803 & 14.491 & 112.282 & 95.787 & 91.885 & -9.836 & -3.484 & 14.862 & -0.220 & 16.007 & 0.366 \\
\hline YAQWEU & 9.284 & 9.309 & 11.899 & 102.136 & 93.599 & 108.409 & 8.350 & 9.402 & 12.101 & 102.988 & 92.872 & 97.687 & -10.060 & 1.002 & 1.698 & 0.834 & -0.777 & -9.890 \\
\hline YAZZAB & 10.033 & 10.147 & 10.431 & 67.151 & 62.715 & 72.064 & 10.782 & 9.547 & 10.338 & 68.679 & 62.058 & 68.014 & 7.465 & -5.913 & -0.892 & 2.275 & -1.048 & -5.620 \\
\hline YENSIT & 11.218 & 11.680 & 15.360 & 93.422 & 110.575 & 90.951 & 11.460 & 12.048 & 15.089 & 103.174 & 113.826 & 76.521 & 2.157 & 3.151 & -1.764 & 10.439 & 2.940 & -15.866 \\
\hline YORFOA & 8.090 & 11.676 & 14.803 & 90.000 & 121.506 & 90.000 & 9.530 & 11.147 & 13.843 & 90.000 & 113.261 & 90.000 & 17.800 & -4.531 & -6.485 & 0.000 & -6.786 & 0.000 \\
\hline YUCXAW & 11.093 & 11.266 & 12.642 & 109.250 & 95.430 & 105.620 & 11.998 & 12.263 & 10.211 & 101.571 & 99.496 & 88.613 & 8.158 & 8.850 & -19.230 & -7.029 & 4.261 & -16.102 \\
\hline ZAXWOK & 17.429 & 8.932 & 18.443 & 90.000 & 112.490 & 90.000 & 17.837 & 8.602 & 20.069 & 90.000 & 119.418 & 90.000 & 2.341 & -3.695 & 8.816 & 0.000 & 6.159 & 0.000 \\
\hline ZOXGAU & 14.146 & 18.003 & 5.372 & 90.000 & 90.000 & 90.000 & 14.821 & 18.283 & 5.277 & 90.000 & 90.000 & 90.000 & 4.772 & 1.555 & -1.768 & 0.000 & 0.000 & 0.000 \\
\hline
\end{tabular}




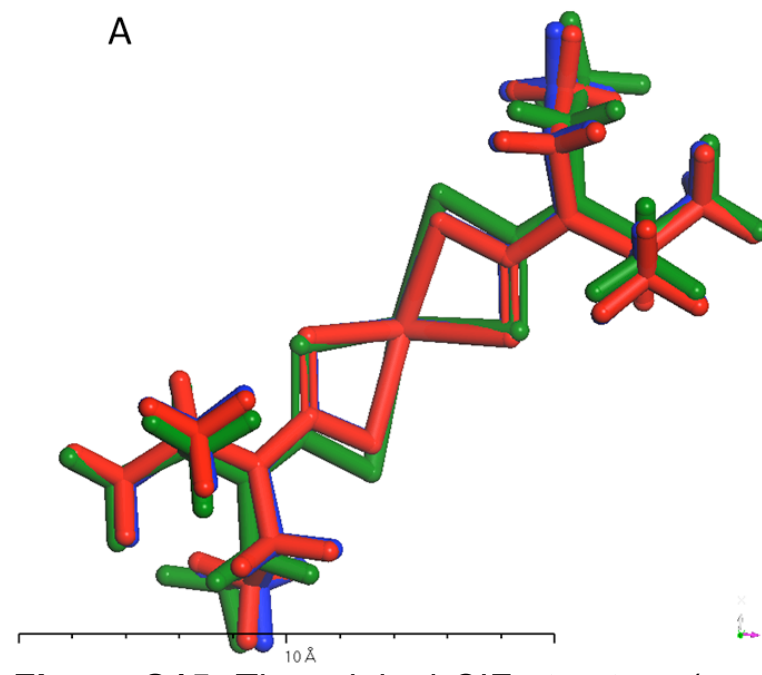

B

Figure S15. The original CIF structure (green) overlaid with the geometry optimized structures using the constrained (red) and unconstrained (blue) unit cells for (A) 1a (NAYNUW01) and (B) 2a (NUNXOK).

Table S2. Bond lengths and angles for 1a (NAYNUW01).

\begin{tabular}{lll}
\hline optimization type & angle $\left(\mathbf{S}_{1}-\mathbf{P b}-\mathbf{S}_{1}{ }^{\circ},{ }^{\circ}\right)$ & length $(\mathbf{S}-\mathbf{P b}, \mathbf{A})$ \\
\hline unoptimized & 138.744 & 2.837 \\
constrained unit cell & 115.830 & 2.245 \\
unconstrained unit cell & 113.986 & 2.234 \\
\hline
\end{tabular}

Table S3. Bond lengths and angles for $\mathbf{2 a}$ (NUNXOK).

\begin{tabular}{lll}
\hline optimization type & angle (O-Pb-O', $\left.{ }^{\circ}\right)$ & length $(\mathbf{O}-\mathbf{P b}, \mathbf{A})$ \\
\hline unoptimized & 93.609 & 2.262 \\
constrained unit cell & 106.614 & 2.033 \\
unconstrained unit cell & 118.456 & 2.012 \\
\hline
\end{tabular}


VIII. Morphology Prediction

A. Crystal Graphs: Impact of Varying the Calculation Radius

Table S4. Predicted aspect ratios (AR) for 1a (NAYNUW01) using different calculation radii in the crystal graph generation.

\begin{tabular}{ccc}
\hline $\begin{array}{c}\text { calc. radius } \\
\text { (\# of unit } \\
\text { cells) }\end{array}$ & $\begin{array}{c}\text { minimum energy } \\
\text { limit (kcal/mol) }\end{array}$ & $\begin{array}{c}\text { aspect } \\
\text { ratio }\end{array}$ \\
\hline 1 & -0.596 & 4.672 \\
1.5 & -0.596 & 4.190 \\
2 & -0.596 & 4.191 \\
1 & none & 4.738 \\
\hline
\end{tabular}

Table S5. Predicted aspect ratios (AR) for $\mathbf{2 a}$ (NUNXOK) using different calculation radii in the crystal graph generation.

\begin{tabular}{ccc}
\hline $\begin{array}{c}\text { calc. radius } \\
\text { (\# of unit } \\
\text { cells) }\end{array}$ & $\begin{array}{c}\text { minimum energy } \\
\text { limit (kcal/mol) }\end{array}$ & $\begin{array}{c}\text { aspect } \\
\text { ratio }\end{array}$ \\
\hline 1 & -0.596 & 4.489 \\
1.5 & -0.596 & 3.957 \\
2 & -0.596 & 3.864 \\
1 & none & 4.392 \\
\hline
\end{tabular}




\section{B. Crystal Graph versus Forcite}
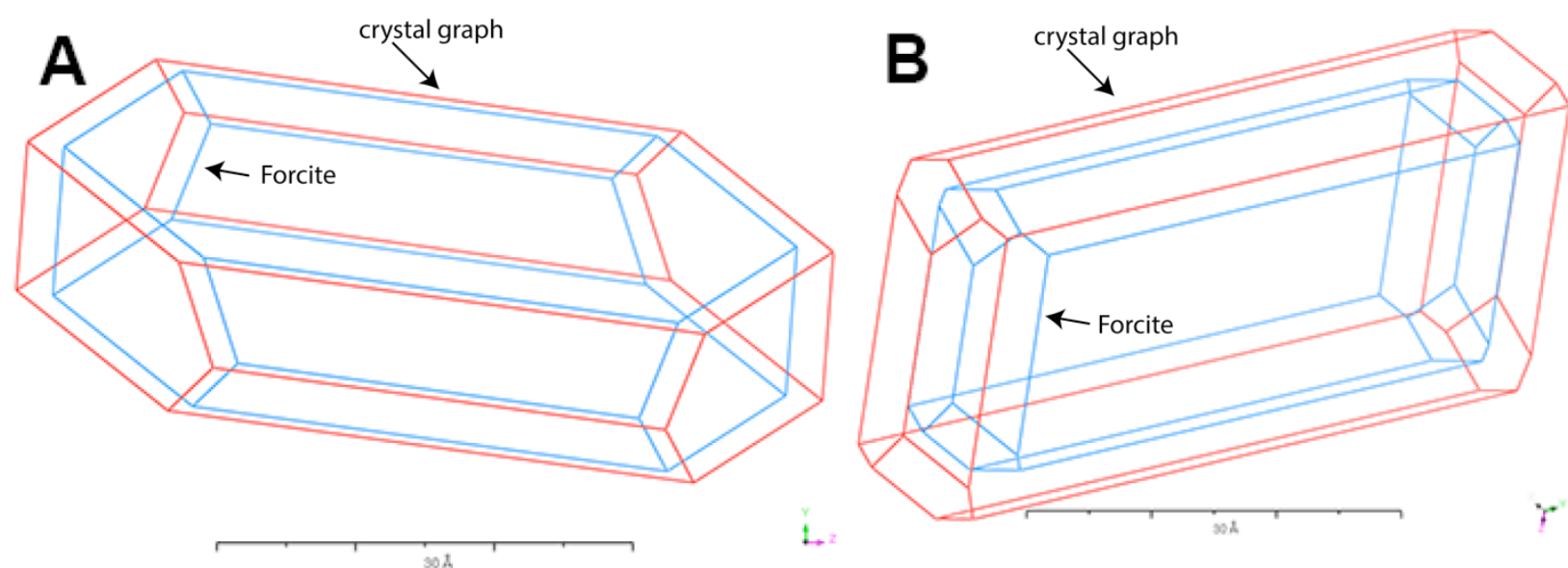

Figure S16. Predicted morphologies for (A) 1a (NAYNUW01) and (B) 2a (NUNXOK) generated by the crystal graph (red) and Forcite (blue) energy methods.

Table S6. Data on the predicted morphology of 1a (NAYNUW01) calculated via the crystal graph and Forcite energy methods.

\begin{tabular}{lllr}
\hline $\begin{array}{c}\text { calc. radius } \\
\text { (\# of unit } \\
\text { cells) }\end{array}$ & $\begin{array}{l}\text { minimum energy } \\
\text { limit (kcal/mol) }\end{array}$ & energy method & $\begin{array}{c}\text { aspect } \\
\text { ratio }\end{array}$ \\
\hline 1 & -0.596 & crystal graph & 4.672 \\
1 & none & crystal graph & 4.738 \\
$\mathrm{n} / \mathrm{a}$ & $\mathrm{n} / \mathrm{a}$ & Forcite & 4.284 \\
\hline
\end{tabular}

Table S7. Data on the predicted morphology of $2 a$ (NUNXOK) calculated via the crystal graph and Forcite energy methods.

\begin{tabular}{lllr}
\hline $\begin{array}{c}\text { calc. radius } \\
\text { (\# of unit } \\
\text { cells) }\end{array}$ & $\begin{array}{l}\text { minimum energy } \\
\text { limit (kcal/mol) }\end{array}$ & energy method & $\begin{array}{c}\text { aspect } \\
\text { ratio }\end{array}$ \\
\hline 1 & -0.596 & crystal graph & 4.489 \\
1 & none & crystal graph & 4.392 \\
$\mathrm{n} / \mathrm{a}$ & $\mathrm{n} / \mathrm{a}$ & Forcite & 3.777 \\
\hline
\end{tabular}




\section{Computed Aspect Ratios}

Table S8. Predicted aspect ratios (AR) for all 184 compounds using either the BFDH or AE methods. The energy of crystallization $\left(E_{\text {latt }}\right)$ is also provided.

\begin{tabular}{cccc}
\hline ComPOUnd & BFDH AR & AE AR & $\mathbf{E}_{\text {latt }}$ \\
\hline NOGQOQ & 1.697 & 1.402 & -482.154 \\
NONXOE & 1.446 & 1.413 & -190.608 \\
PUQVUT & 2.220 & 1.475 & -332.123 \\
POGDOE & 1.680 & 1.495 & -194.920 \\
XOPDUB & 1.745 & 1.535 & -100.042 \\
XISSAT & 1.516 & 1.551 & -257.687 \\
NUFQEL02 & 1.379 & 1.557 & -158.469 \\
TIWCUX & 1.486 & 1.560 & -344.034 \\
PAQVIN & 1.358 & 1.570 & -2219.252 \\
GAGXES & 1.464 & 1.582 & -197.131 \\
FOPJIE & 1.533 & 1.613 & -526.131 \\
HOXQOB & 1.506 & 1.626 & -5418.562 \\
RABKEK & 1.569 & 1.678 & -86.882 \\
BOJXUU & 1.399 & 1.679 & -192.095 \\
JAWHAR & 1.845 & 1.683 & -1930.351 \\
OKIMUR & 1.616 & 1.693 & -135.154 \\
WOCYUJ & 1.633 & 1.694 & -374.918 \\
GAGCIC & 1.501 & 1.696 & -266.082 \\
ACAHUI & 1.958 & 1.707 & -191.651 \\
HEDNIO & 1.521 & 1.708 & -284.383 \\
HUGVUB & 1.585 & 1.712 & -109.747 \\
ULAWAG & 1.920 & 1.727 & -237.655 \\
MIFSIE02 & 1.759 & 1.734 & -732.889 \\
RAQFAQ01 & 2.005 & 1.737 & -287.997 \\
DURGON & 1.692 & 1.739 & -225.414 \\
PUHYAT & 1.882 & 1.744 & -114.721 \\
NUWHUJ & 1.436 & 1.747 & -298.696 \\
FOHFEO & 2.134 & 1.749 & -518.689 \\
JIBZAV & 1.611 & 1.752 & -122.446 \\
ATACUT & 2.440 & 1.759 & -352.935 \\
OJAMIW & 1.344 & 1.759 & -237.045 \\
KEGZEB & 1.809 & 1.764 & -116.655 \\
QEYSET & 1.892 & 1.773 & -686.756 \\
AJIMUB & 2.301 & 1.800 & -310.370 \\
XAGKIB & 1.684 & 1.811 & -288.893 \\
XIRCEH & 1.807 & 1.812 & -496.810 \\
QOKHAZ & 1.502 & 1.813 & -214.771 \\
NOMJUV & 1.653 & 1.828 & -133.895 \\
\hline & & & \\
\hline
\end{tabular}




\begin{tabular}{|c|c|c|c|}
\hline Compound & BFDH AR & AE AR & $E_{\text {latt }}$ \\
\hline MONLIK & 1.481 & 1.829 & -474.629 \\
\hline DURJEG & 2.243 & 1.830 & -115.817 \\
\hline XIJFOL & 2.046 & 1.831 & -225.098 \\
\hline YAZZAB & 1.409 & 1.836 & -92.223 \\
\hline GIMPUN & 1.456 & 1.852 & -311.291 \\
\hline QEYSIX & 1.629 & 1.878 & -408.346 \\
\hline NAPVOR & 2.008 & 1.880 & -251.106 \\
\hline HIQHEV & 1.528 & 1.881 & -254.023 \\
\hline IVEFIY & 1.475 & 1.890 & -118.475 \\
\hline YORFOA & 1.499 & 1.891 & -90.354 \\
\hline CIJQES & 1.590 & 1.900 & -120.079 \\
\hline SUXZOB & 1.536 & 1.904 & -94.673 \\
\hline GOLMIE & 1.377 & 1.911 & -510.610 \\
\hline LIZGAD & 1.867 & 1.914 & -328.793 \\
\hline PASBAM & 2.030 & 1.918 & -150.058 \\
\hline ABUTEX01 & 1.598 & 1.920 & -399.779 \\
\hline CIJQIW01 & 1.590 & 1.922 & -123.930 \\
\hline CIJQIW & 1.588 & 1.928 & -121.617 \\
\hline NAKGIP & 1.657 & 1.933 & -585.933 \\
\hline COCLAI & 1.797 & 1.943 & -331.173 \\
\hline DAMTIV & 1.632 & 1.946 & -281.407 \\
\hline KEGZAX & 2.067 & 1.947 & -186.147 \\
\hline KUJCIB & 1.789 & 1.953 & -218.562 \\
\hline YUCXAW & 1.575 & 1.953 & -234.759 \\
\hline ILOBUH & 1.557 & 1.966 & -190.903 \\
\hline HUGWAI & 1.983 & 1.980 & -472.612 \\
\hline TOXPEB & 1.692 & 1.986 & -484.999 \\
\hline NEDVAU & 1.682 & 1.990 & -215.312 \\
\hline TASCIZ & 1.862 & 1.998 & -217.138 \\
\hline PUQVON & 2.470 & 2.007 & -148.271 \\
\hline LAJCAC & 1.425 & 2.020 & -199.592 \\
\hline SAHSIE & 2.167 & 2.066 & -286.836 \\
\hline IJONEB & 2.245 & 2.073 & -138.101 \\
\hline BAMNUZ & 1.558 & 2.074 & -421.629 \\
\hline GOLMOK & 2.106 & 2.095 & -156.089 \\
\hline TOJPIR & 1.479 & 2.096 & -64.005 \\
\hline GUWCOR & 1.622 & 2.098 & -420.570 \\
\hline SAHRID & 1.451 & 2.101 & -172.397 \\
\hline YAGLAT01 & 1.632 & 2.107 & -424.265 \\
\hline NIPFAU & 2.190 & 2.127 & -530.545 \\
\hline
\end{tabular}




\begin{tabular}{|c|c|c|c|}
\hline Compound & BFDH AR & AE AR & $E_{\text {latt }}$ \\
\hline VEJNIH & 1.432 & 2.130 & -641.187 \\
\hline IHEMUE & 2.129 & 2.138 & -168.430 \\
\hline FIMDUB & 1.492 & 2.140 & -299.211 \\
\hline YAQWEU & 1.810 & 2.144 & -130.934 \\
\hline WAJQON01 & 2.096 & 2.152 & -388.375 \\
\hline OZCDPB & 1.497 & 2.152 & -148.090 \\
\hline SIFKAU & 1.461 & 2.159 & -207.359 \\
\hline QAFPIY & 2.323 & 2.161 & -472.699 \\
\hline LIZFUW & 1.722 & 2.161 & -137.412 \\
\hline OSAKEZ & 1.809 & 2.183 & -265.192 \\
\hline XIRBUW & 1.591 & 2.202 & -481.014 \\
\hline TAMBUF & 1.704 & 2.215 & -192.221 \\
\hline JUCCEP02 & 1.545 & 2.241 & -102.614 \\
\hline WABQEX & 1.633 & 2.248 & -97.892 \\
\hline МТСВРВ & 2.275 & 2.249 & -322.139 \\
\hline WALQIK & 1.745 & 2.251 & -115.426 \\
\hline KUQFAE & 1.578 & 2.253 & -68.185 \\
\hline ULAVUZ & 1.818 & 2.257 & -111.134 \\
\hline LERQAB & 1.482 & 2.266 & -215.041 \\
\hline GOLMEA & 1.641 & 2.267 & -69.371 \\
\hline TUMFAJ & 2.230 & 2.280 & -394.713 \\
\hline JITBEU & 1.682 & 2.285 & -108.451 \\
\hline PILYAK & 2.293 & 2.299 & -126.801 \\
\hline VEWREU & 2.112 & 2.302 & -144.970 \\
\hline KAMKAL & 2.046 & 2.312 & -390.429 \\
\hline GICKUZ & 2.160 & 2.325 & -186.159 \\
\hline CEVLEV & 1.774 & 2.331 & -174.670 \\
\hline IWOVAR & 1.549 & 2.356 & -166.916 \\
\hline GODWIG & 1.805 & 2.358 & -121.145 \\
\hline HAGSOX & 1.687 & 2.360 & -209.770 \\
\hline FOFCEI & 2.294 & 2.371 & -132.992 \\
\hline BOJXOO & 1.686 & 2.380 & -80.782 \\
\hline DAXMAR & 2.223 & 2.400 & -392.430 \\
\hline BAVDAE & 1.470 & 2.402 & -377.727 \\
\hline NIXTIX & 1.514 & 2.410 & -84.083 \\
\hline AKOHOY & 2.050 & 2.412 & -122.249 \\
\hline WUXGAY & 1.571 & 2.414 & -610.834 \\
\hline OXURUV & 1.581 & 2.431 & -263.830 \\
\hline ACASON & 1.657 & 2.451 & -397.724 \\
\hline
\end{tabular}




\begin{tabular}{|c|c|c|c|}
\hline Compound & BFDH AR & AE AR & $E_{\text {latt }}$ \\
\hline ERIGES & 1.823 & 2.456 & -104.083 \\
\hline RAQQOP & 1.796 & 2.485 & -112.317 \\
\hline ACASIH & 2.591 & 2.488 & -143.650 \\
\hline YENSIT & 1.896 & 2.507 & -167.440 \\
\hline XANCOG & 1.519 & 2.515 & -176.663 \\
\hline WILNOV & 1.943 & 2.535 & -105.816 \\
\hline WIMKIN & 1.885 & 2.555 & -405.997 \\
\hline UDIKOI & 2.033 & 2.602 & -201.759 \\
\hline BAHKEC & 2.146 & 2.608 & -139.839 \\
\hline FIHCUV & 1.937 & 2.637 & -130.044 \\
\hline QIBSIE & 1.437 & 2.639 & -371.833 \\
\hline XUZZAU & 2.173 & 2.673 & -107.067 \\
\hline BOSDOD & 1.412 & 2.685 & -92.347 \\
\hline NADVEV & 2.388 & 2.697 & -149.321 \\
\hline MOXYOO & 1.427 & 2.714 & -252.396 \\
\hline ZOXGAU & 2.299 & 2.729 & -125.394 \\
\hline JUCCEP & 1.529 & 2.733 & -95.550 \\
\hline HERHUH & 1.530 & 2.767 & 103.175 \\
\hline WITQOF & 1.602 & 2.776 & -136.827 \\
\hline GOPYIT & 1.529 & 2.788 & -234.969 \\
\hline JADJIH & 2.005 & 2.795 & -166.916 \\
\hline WALQOQ & 1.732 & 2.798 & -102.614 \\
\hline DOBZID10 & 2.080 & 2.802 & -116.562 \\
\hline CIQGUE & 2.060 & 2.808 & -168.869 \\
\hline SUCNUZ & 1.781 & 2.811 & -152.337 \\
\hline VAMWIP01 & 2.290 & 2.821 & -186.848 \\
\hline BUMMOL & 2.150 & 2.880 & -71.538 \\
\hline WATSEP & 1.871 & 2.882 & -247.983 \\
\hline DUPXAO & 1.674 & 2.949 & -130.479 \\
\hline HEFHAB & 1.934 & 3.021 & -118.299 \\
\hline PIGWAE & 1.684 & 3.029 & -115.757 \\
\hline RASRAE & 2.004 & 3.061 & -277.786 \\
\hline KILMEX & 1.832 & 3.082 & -333.810 \\
\hline WAQBIZ & 2.081 & 3.210 & -85.117 \\
\hline $\mathrm{KUJCOH}$ & 1.864 & 3.244 & -125.533 \\
\hline LEWPIM & 3.365 & 3.272 & -117.723 \\
\hline BEQWUQ & 1.853 & 3.295 & -262.680 \\
\hline GOJGUI & 2.586 & 3.348 & -179.926 \\
\hline VOCHIF & 3.433 & 3.355 & -404.253 \\
\hline
\end{tabular}




\begin{tabular}{|c|c|c|c|}
\hline Compound & BFDH AR & AE AR & $E_{\text {latt }}$ \\
\hline OSAKAV & 2.431 & 3.368 & -89.056 \\
\hline HUGWEM & 2.371 & 3.370 & -115.731 \\
\hline XAGKEX & 1.897 & 3.426 & -85.648 \\
\hline HABGAU & 2.316 & 3.507 & -121.372 \\
\hline FAZFIX & 2.852 & 3.531 & -225.078 \\
\hline ABIBUJ & 1.591 & 3.581 & -126.746 \\
\hline EFUBAJ & 2.316 & 3.659 & -508.622 \\
\hline WICSIK & 1.726 & 3.763 & -164.158 \\
\hline QOPJAH & 2.350 & 3.764 & -450.979 \\
\hline AJOGOW & 2.548 & 3.833 & -571.539 \\
\hline KUQFAE01 & 2.560 & 3.967 & -118.822 \\
\hline CIJQOC & 3.863 & 4.088 & -237.837 \\
\hline NUNXOK & 2.621 & 4.489 & -199.211 \\
\hline WACCUA & 2.583 & 4.536 & -88.713 \\
\hline NAYNUW01 & 2.471 & 4.672 & -183.969 \\
\hline EFOZUV & 1.855 & 4.796 & -121.356 \\
\hline UCARAT & 1.624 & 5.413 & -210.518 \\
\hline MOXZEF & 1.732 & 5.540 & -92.778 \\
\hline ETUJOT & 1.899 & 7.123 & -322.391 \\
\hline ZAXWOK & 1.404 & failed $^{\dagger}$ & -224.969 \\
\hline NETVIR & 1.443 & failed $^{\dagger}$ & -303.724 \\
\hline MAWNON & 1.583 & failed $^{\dagger}$ & -297.911 \\
\hline DODMUF & 1.614 & failed $^{\dagger}$ & -3516.158 \\
\hline QUXQAC & 1.635 & failed $^{\dagger}$ & -245.176 \\
\hline MAWPAB & 1.753 & failed $^{\dagger}$ & -515.599 \\
\hline TATREM & 1.921 & failed $^{\dagger}$ & -416.802 \\
\hline BURPIO & 1.954 & failed $^{\dagger}$ & -399.174 \\
\hline AYUDON & 1.957 & failed $^{\dagger}$ & -190.572 \\
\hline
\end{tabular}



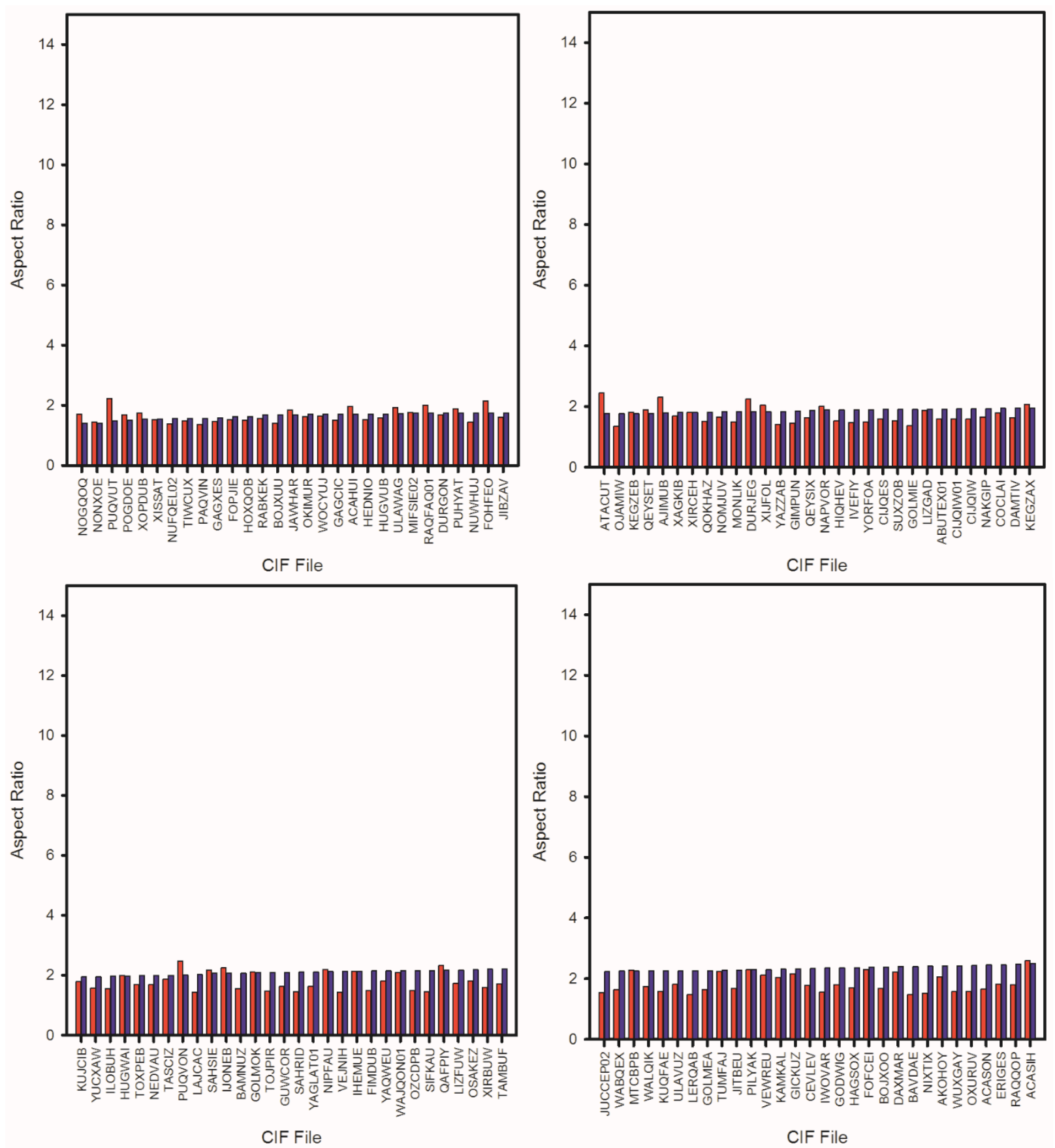

Figure S17A. Plot of the predicted aspect ratios using $A E$ (blue) and BFDH (red) methods versus CIF name for all compounds with predicted stable morphologies (numerical values can be found in Table S8.) 

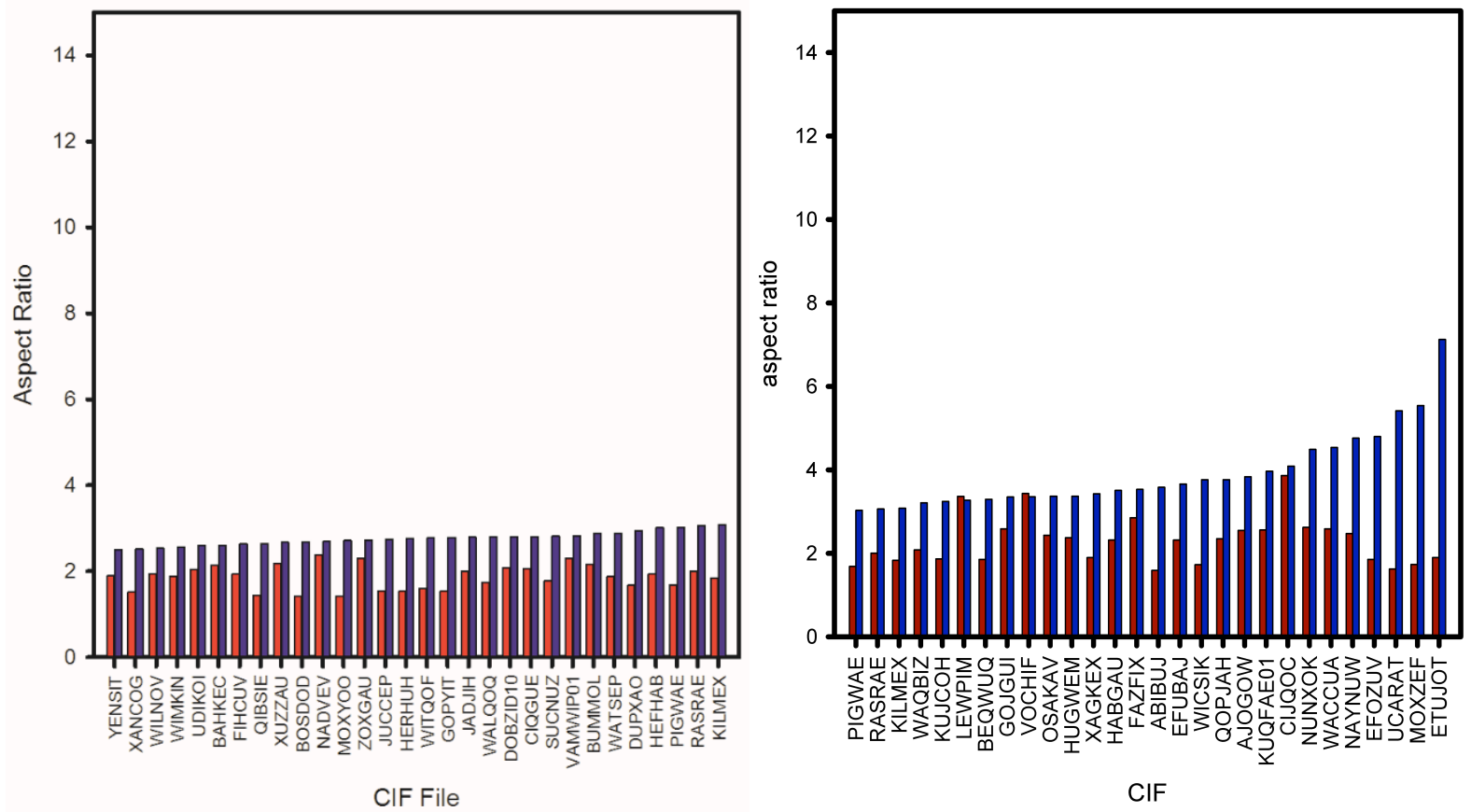

Figure S17B. Plot of the predicted aspect ratios using AE (blue) and BFDH (red) methods versus CIF file name for all compounds with predicted stable morphologies (numerical values can be found in Table S8.) 
IX. A Closer Look at the Top 5\% of Attachment Energy-predicted Aspect Ratios.

Table S9. Predicted aspect ratios (AR) and lattice energies $\left(E_{l a t t}\right)$ for the top $5 \%$.

\begin{tabular}{ccccccc}
\hline CIF File & $\begin{array}{c}\text { AE } \\
\text { aspect } \\
\text { ratio }\end{array}$ & $\begin{array}{c}\mathbf{E}_{\text {latt }} \\
\mathbf{( k c a l / m o l )}\end{array}$ & $\begin{array}{c}\text { \# of } \\
\text { unique } \\
\text { faces }\end{array}$ & $\begin{array}{c}\text { Normalized } \\
\mathbf{E}_{\text {latt }} \\
(\mathbf{k c a l} / \mathbf{m o l})\end{array}$ & $\begin{array}{c}\mathbf{E}_{\text {attach }} \\
\text { smallest } \\
\text { face } \\
\text { (kcal/mol) }\end{array}$ & $\begin{array}{c}\mathbf{E}_{\text {attach largest }} \\
\text { face } \\
\text { (kcal/mol) }\end{array}$ \\
\hline KUQFAE01 & 3.967 & -118.822 & 3 & -29.706 & -84.308 & -24.667 \\
CIJQOC & 4.088 & -237.837 & 3 & -59.459 & -99.244 & -42.581 \\
NUNXOK (2a) & 4.489 & -199.211 & 5 & -33.202 & -149.674 & -35.186 \\
WACCUA & 4.536 & -88.713 & 5 & -44.357 & -67.643 & -16.099 \\
NAYNUW01 (1a) & 4.672 & -183.969 & 3 & -30.662 & -105.712 & -32.650 \\
EFOZUV & 4.796 & -121.356 & 5 & -60.678 & -73.338 & -16.416 \\
UCARAT & 5.413 & -210.518 & 14 & -52.630 & -176.095 & -33.289 \\
MOXZEF & 5.540 & -92.778 & 4 & -23.195 & -83.589 & -15.809 \\
ETUJOT & 7.123 & -322.391 & 5 & -26.866 & -281.629 & -27.367 \\
\hline
\end{tabular}

${ }^{a}$ Normalized $E_{\text {latt }}$ is the energy per molecule in the unit cell. Fragmented molecules in the unit cell were completed for the calculations. 


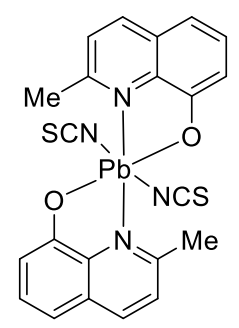

KUQFAE01

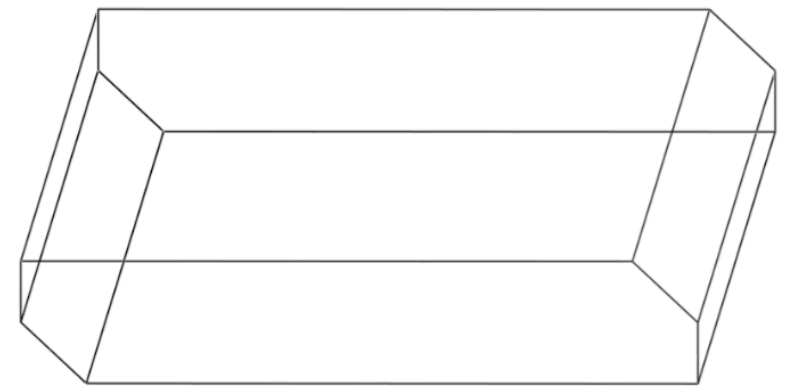

Figure S18. Molecular diagram and predicted morphology for KUQFAE01. ${ }^{7}$

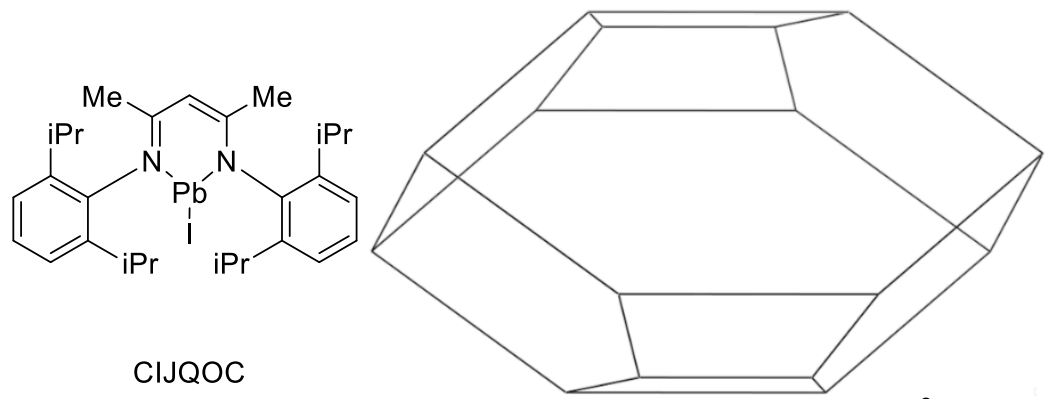

Figure S19. Molecular diagram and predicted morphology for CIJQOC. ${ }^{8}$
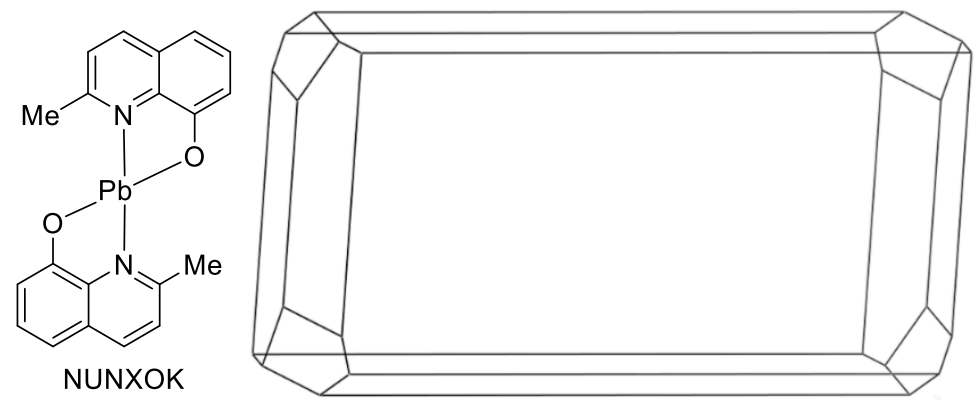

Figure S20. Molecular diagram and predicted morphology for NUNXOK (2a) ${ }^{4}$ 

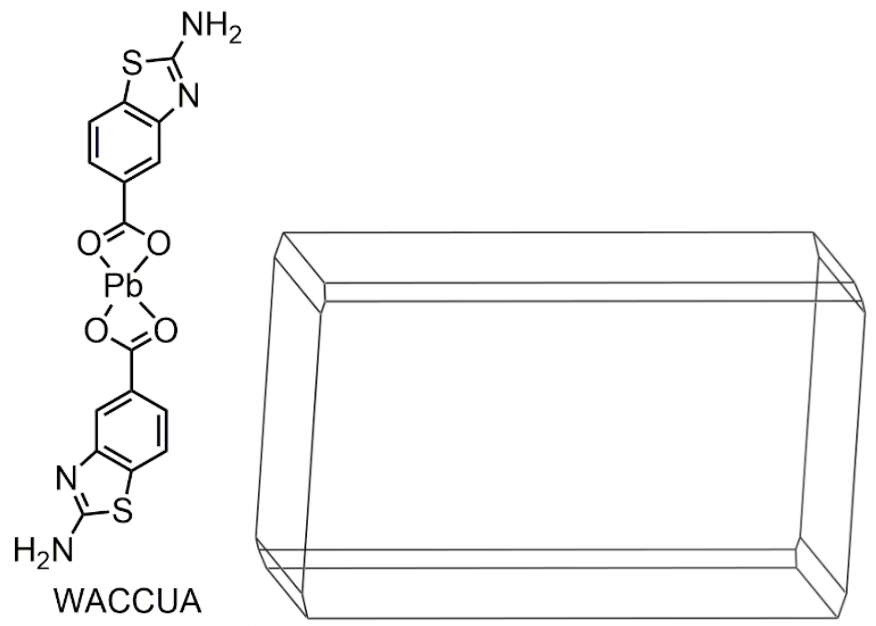

Figure S21. Molecular diagram and predicted morphology for WACCUA. ${ }^{9}$

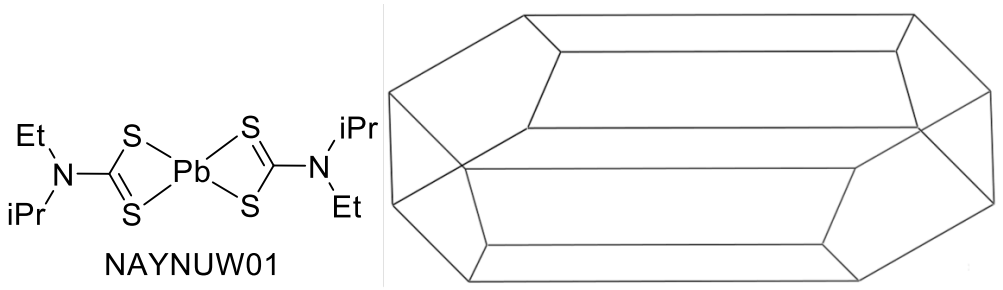

Figure S22. Molecular diagram and predicted morphology for NAYNUW01 (1a). ${ }^{2,3}$

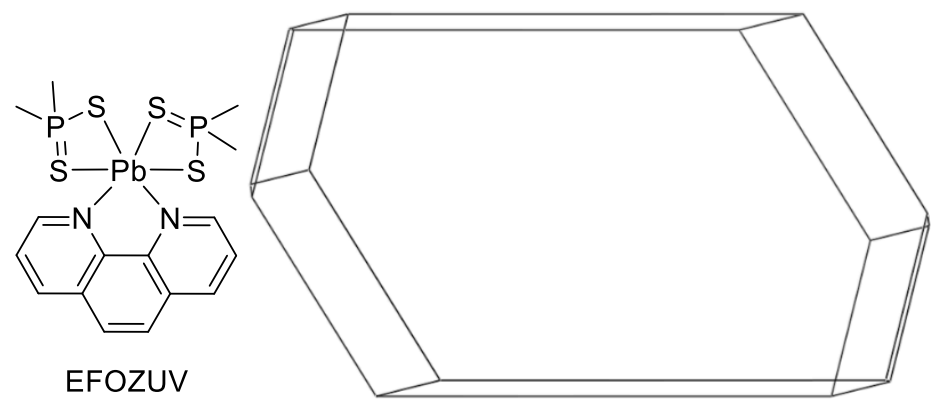

Figure S23. Molecular diagram and predicted morphology for EFOZUV. ${ }^{10}$
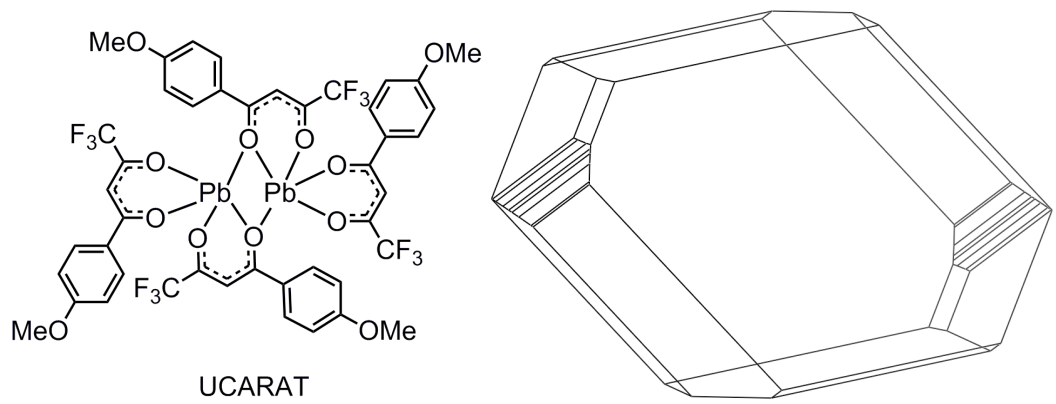

Figure S24. Molecular diagram and predicted morphology for UCARAT. ${ }^{11}$ 

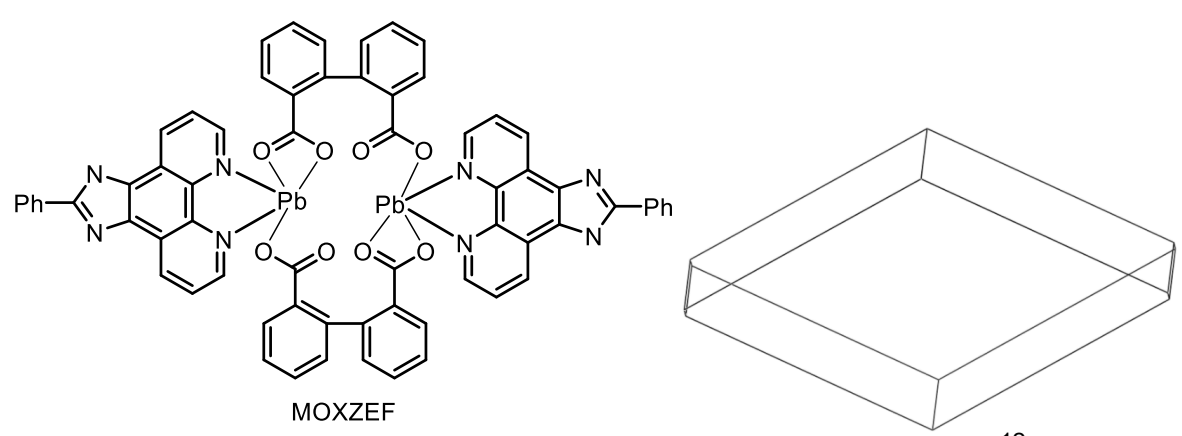

Figure S25. Molecular diagram and predicted morphology for MOXZEF. ${ }^{12}$

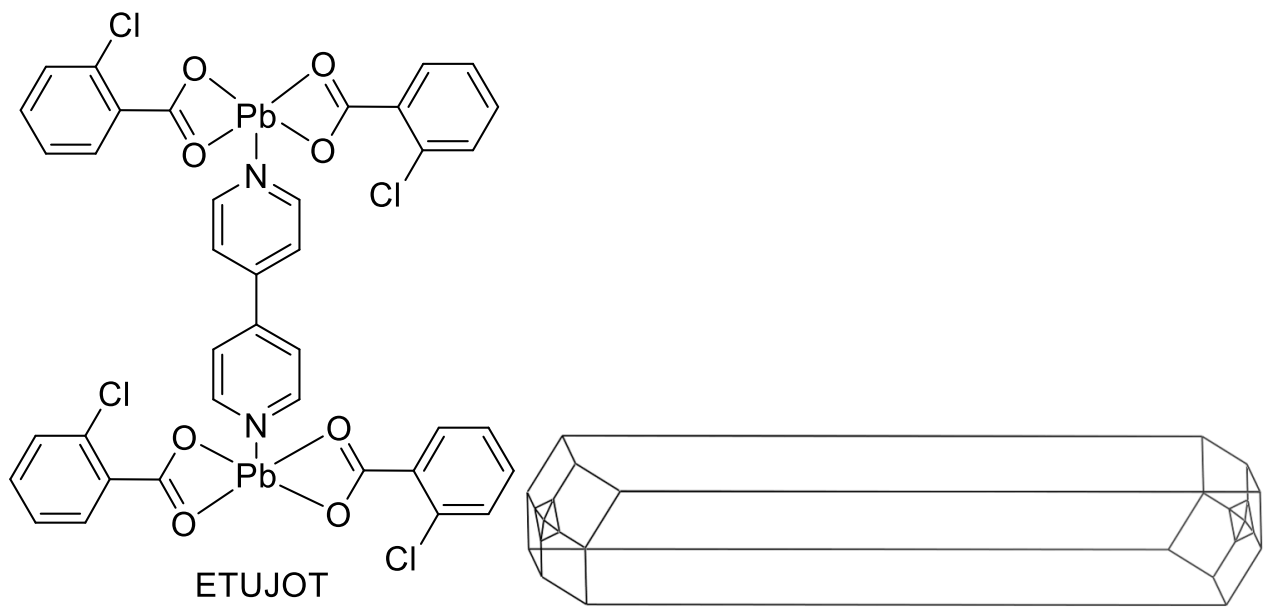

Figure S26. Molecular diagram and predicted morphology for ETUJOT. ${ }^{13}$ 


\section{Impact of Electron Delocalization on Morphology Prediction}

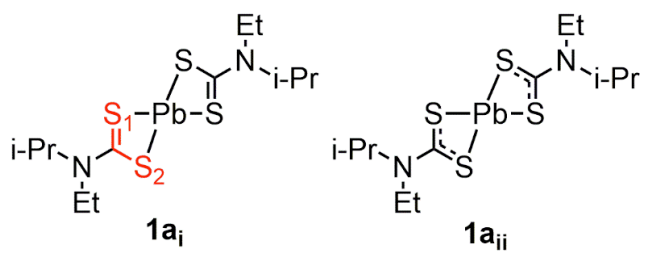

Figure S27. Resonance structures for 1a (NAYNUW01) used in morphology calculations.

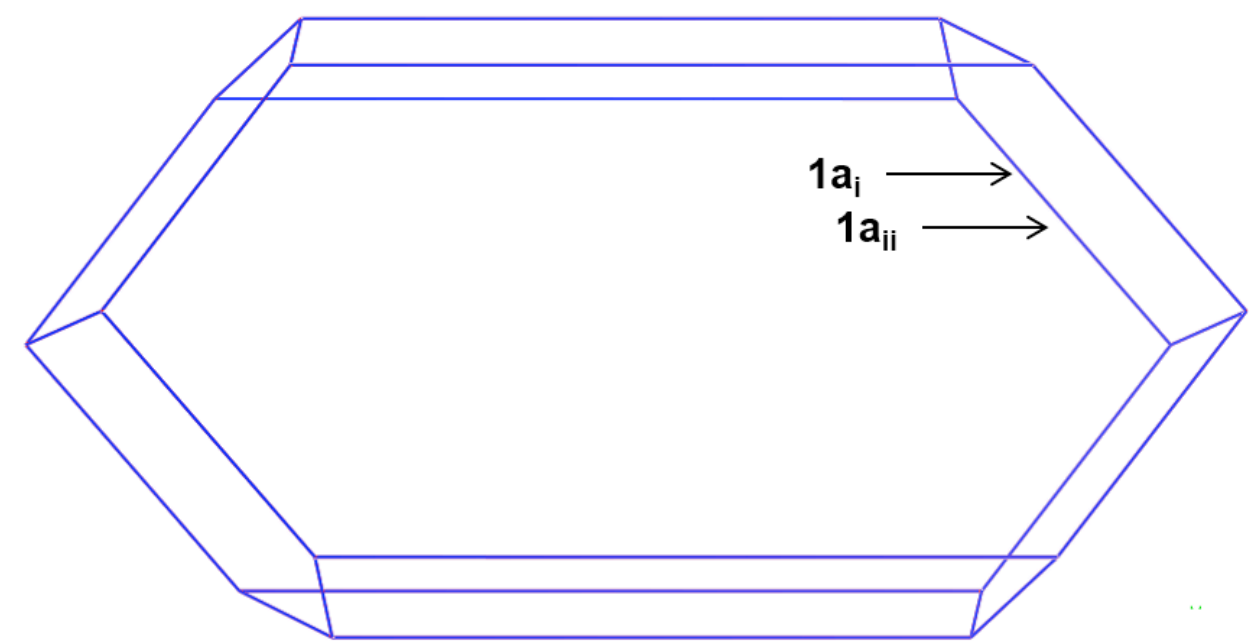

Figure S28. Predicted morphologies for resonance structures $\mathbf{1} \mathbf{a}_{\mathbf{i}}(\mathrm{red})$ and $\mathbf{1} \mathbf{a}_{\mathrm{ii}}$ (blue). Note that the two morphologies are nearly identical.

Table S10. Bond lengths and predicted aspect ratios (AR) for unoptimized 1a (NAYNUW01) and optimized $1 \mathbf{a}_{\mathrm{i}}$ and $1 \mathbf{a}_{\mathrm{ii}}$.

\begin{tabular}{cccc}
\hline & $\mathrm{S}_{1}-\mathrm{C}(\AA)$ & $\mathrm{C}-\mathrm{S}_{2}(\AA)$ & $\mathrm{AR}$ \\
\hline unoptimized & 1.720 & 1.728 & $\mathrm{n} / \mathrm{a}$ \\
$\mathbf{1 a}_{\mathbf{i}}$ & 1.507 & 1.506 & 4.672 \\
$\mathbf{1 a}_{\mathrm{ii}}$ & 1.507 & 1.506 & 4.672 \\
\hline
\end{tabular}




\section{Gel Formation Experiments}

Gel screening 1a-1h and 2a-2b. A known amount of lead complex ( 1-10 mg) was weighed into an $8 \mathrm{~mL}$ vial. Then, $0.3-0.5 \mathrm{~mL}$ of indicated solvent was added and the vial was capped. If the material did not immediately dissolve, the mixture was heated and/or sonicated to form a solution. If the compound did not go into solution upon heating, additional solvent was added until all material dissolved upon heating. The vial was heated, and then cooled with approximately $20 \mathrm{~s}(\mathbf{1 a}-\mathbf{1 d})$ to $30 \mathrm{~s}(\mathbf{2 a - 2 b})$ of sonication in a $\mathrm{H}_{2} \mathrm{O}$ bath (near ambient temperature). After standing undisturbed at rt for at least $10 \mathrm{~min}$, the vial was inverted to test for gelation.

Table S11. Gel screening for 1a-1h. ${ }^{a}$

\begin{tabular}{|c|c|c|c|c|c|c|c|c|}
\hline Solvent & $1 a$ & $1 b$ & $1 c$ & $1 d$ & $1 e$ & $1 f$ & $1 \mathrm{~g}$ & $1 \mathrm{~h}$ \\
\hline acetone & sol & ppt & sol & gel & sol & gel & gel & ppt \\
\hline EtOAc & ppt & ppt & sol & gel & sol & sol & gel & gel \\
\hline $\mathrm{MeOH}$ & ppt & ppt & cryst & gel & gel & gel & gel & ppt \\
\hline toluene & sol & ppt & sol & gel & sol & sol & sol & sol \\
\hline THF & sol & sol & sol & gel & sol & sol & sol & sol \\
\hline iPrOH & ppt & ppt & cryst & gel & gel & gel & gel & ppt \\
\hline $\mathrm{CH}_{2} \mathrm{Cl}_{2}$ & sol & sol & sol & gel & sol & sol & sol & sol \\
\hline $\mathrm{H}_{2} \mathrm{O}$ & ppt & ppt & ppt & ppt & ppt & ppt & ppt & ppt \\
\hline $\mathrm{EtOH}$ & ppt & ppt & sol & gel & gel & gel & gel & ppt \\
\hline hexanes & $\mathrm{ppt}$ & ppt & sol & ppt & ppt & ppt & ppt & ppt \\
\hline $\begin{array}{c}\text { paint } \\
\text { thinner }\end{array}$ & ppt & ppt & sol & gel & gel & gel & gel & ppt \\
\hline MEK $^{\mathrm{b}}$ & sol & cryst & sol & gel & sol & sol & gel & gel \\
\hline
\end{tabular}

a) $\mathrm{sol}=$ solution; $\mathrm{ppt}=$ precipitate cryst $=$ crystals. b) $\mathrm{MEK}=$ methyl ethyl ketone.

Table S12. Gel screening for $2 \mathbf{a}-\mathbf{b} .^{a}$

\begin{tabular}{ccc}
\hline Solvent & $\mathbf{2 a}$ & $\mathbf{2 b}$ \\
\hline acetone & ppt & ppt \\
EtOAc & ppt & ppt \\
$1 \mathrm{M} \mathrm{NaOH}$ & ppt & gel \\
$\mathrm{EtOH} / 1 \mathrm{M} \mathrm{NaOH}$ & gel \\
$\mathrm{MeOH} / 1 \mathrm{M} \mathrm{NaOH}$ & ppt & gel \\
$\mathrm{H}_{2} \mathrm{O}$ & ppt & ppt \\
$\mathrm{MeOH} / \mathrm{H}_{2} \mathrm{O}^{\mathrm{c}}$ & ppt & ppt \\
\hline
\end{tabular}

a) sol = solution; ppt = precipitate. b) 1:1 mixture of solvents. c) 2:1 mixture of solvents

Determining the critical gel concentration (cgc) of 1d-1f. The cgc was determined by adding a known amount of gelator (1d-1f) $(\sim 1-10 \mathrm{mg})$ into an $8 \mathrm{~mL}$ vial. To the vial, $0.3 \mathrm{~mL}$ solvent was added. The vial was capped, heated to dissolve the solid, and cooled with approximately $20 \mathrm{~s}$ of sonication in a $\mathrm{H}_{2} \mathrm{O}$ bath (near ambient temperature). After standing undisturbed at rt for at least $10 \mathrm{~min}$, the vial was inverted to test for gelation. If the resulting gel was stable to inversion, additional solvent $(0.1 \mathrm{~mL})$ was added, and the heating, sonication, and 
resting steps repeated. Additional $0.1 \mathrm{~mL}$ increments of solvent were added until the gel was no longer stable to inversion. The reported cgcs are averages of three experiments.

Determining the cgc of $\mathbf{2 b}$. A known amount of $\mathbf{2 b}(\sim 5-10 \mathrm{mg})$ was placed in an $8 \mathrm{~mL}$ vial. Then, the indicated solvent $(0.5-1.0 \mathrm{~mL})$ was added. The vial was capped and heated to dissolve the solid, then cooled with $\sim 30 \mathrm{~s}$ of sonication in a $\mathrm{H}_{2} \mathrm{O}$ bath (near ambient temperature). After standing undisturbed at rt for at least $10 \mathrm{~min}$, the vial was inverted to test for gelation. If the resulting gel was stable to inversion, additional solvent $(0.1 \mathrm{~mL})$ was added, and the heating, sonication, and resting steps repeated. Additional $0.1 \mathrm{~mL}$ increments of solvent were added until the gel was no longer stable to inversion. The reported cgcs are averages of three experiments.

In an alternative gelation procedure, a known amount of $\mathbf{2 b}$ was weighed out and placed in an $8 \mathrm{~mL}$ vial with $0.5 \mathrm{~mL}$ of EtOH. Then, $50 \mu \mathrm{L}$ of HOAc was added and the vial was capped and shaken to dissolve. Next, $1 \mathrm{~mL}$ of $1 \mathrm{M} \mathrm{NaOH}$ was added in $0.1 \mathrm{~mL}$ increments. Finally, the vial was capped and heated to dissolve, then allowed to cool with $\sim 30 \mathrm{~s}$ of sonication in a water bath (near ambient temperature). The cgc of $\mathbf{2} \mathbf{b}$ was determined by decreasing the amount weighed out until gelation no longer occurred.

Table S13. Cgcs for $1 \mathrm{~d}-1 \mathrm{~h}$ and $\mathbf{2 b}$.

\begin{tabular}{ccccc}
\hline compound & acetone & ethyl acetate & paint thinner & ${\text { basic } \mathrm{H}_{2} \text { O/EtOH }}^{\text {a }}$ \\
\hline 1d & $1.84 \pm 0.07 \mathrm{mM}$ & $1.29 \pm 0.09 \mathrm{mM}$ & $0.77 \pm 0.13 \mathrm{mM}$ & -- \\
$\mathbf{1 e}$ & -- & -- & $4.12 \pm 0.04 \mathrm{mM}$ & -- \\
$\mathbf{1 f}$ & $27.81 \pm 4.30 \mathrm{mM}$ & -- & $1.11 \pm 0.04 \mathrm{mM}$ & -- \\
$\mathbf{1 g}$ & $3.79 \pm 0.33 \mathrm{mM}$ & $5.17 \pm 0.48 \mathrm{mM}$ & $1.46 \pm 0.08 \mathrm{mM}$ & -- \\
$\mathbf{1 h}$ & -- & $3.83 \pm 1.13 \mathrm{mM}$ & -- & - \\
$\mathbf{2 b}$ & -- & -- & -- & $16.1 \pm 0.86 \mathrm{mM}^{\mathrm{a}}$ \\
$\mathbf{2 b}$ & -- & -- & -- & $13.9 \pm 0.30 \mathrm{mM}^{\mathrm{b}}$ \\
\hline
\end{tabular}

a) 1:1 mixture $\mathrm{EtOH} / 1 \mathrm{M} \mathrm{NaOH}$. b) Using the alternative gelation procedure described above. 


\section{Rheological Characterization of Gels}

General Procedure - Rheological measurements were taken on an AR2000ex rheometer (TA Instruments) with a $25 \mathrm{~mm}$ serrated parallel plate. All samples were prepared at a concentration of $\sim 1.5-2 \times$ cgc. Approximately $30 \mathrm{~min}$ after a gel was formed it was loaded onto the plate (unless otherwise noted). The gap was then fixed at 300 to $500 \mu \mathrm{m}$, and a solvent trap was used to limit solvent evaporation. All measurements were performed at $25^{\circ} \mathrm{C}$. Each sample was pre-sheared under a stress of $0.06366 \mathrm{~Pa}$ for $1 \mathrm{~min}$ before conducting the frequency sweep and oscillating stress sweep experiments. All measurements were repeated an average of 3 times to verify reproducibility. The frequency sweep experiment was performed under $0.6 \mathrm{~Pa}$ stress with a frequency range from $0.1-100 \mathrm{rad} / \mathrm{s}$. The oscillating stress sweep experiment was performed at $1 \mathrm{~Hz}$, with a stress range from 0.06366 to $250 \mathrm{~Pa}$.
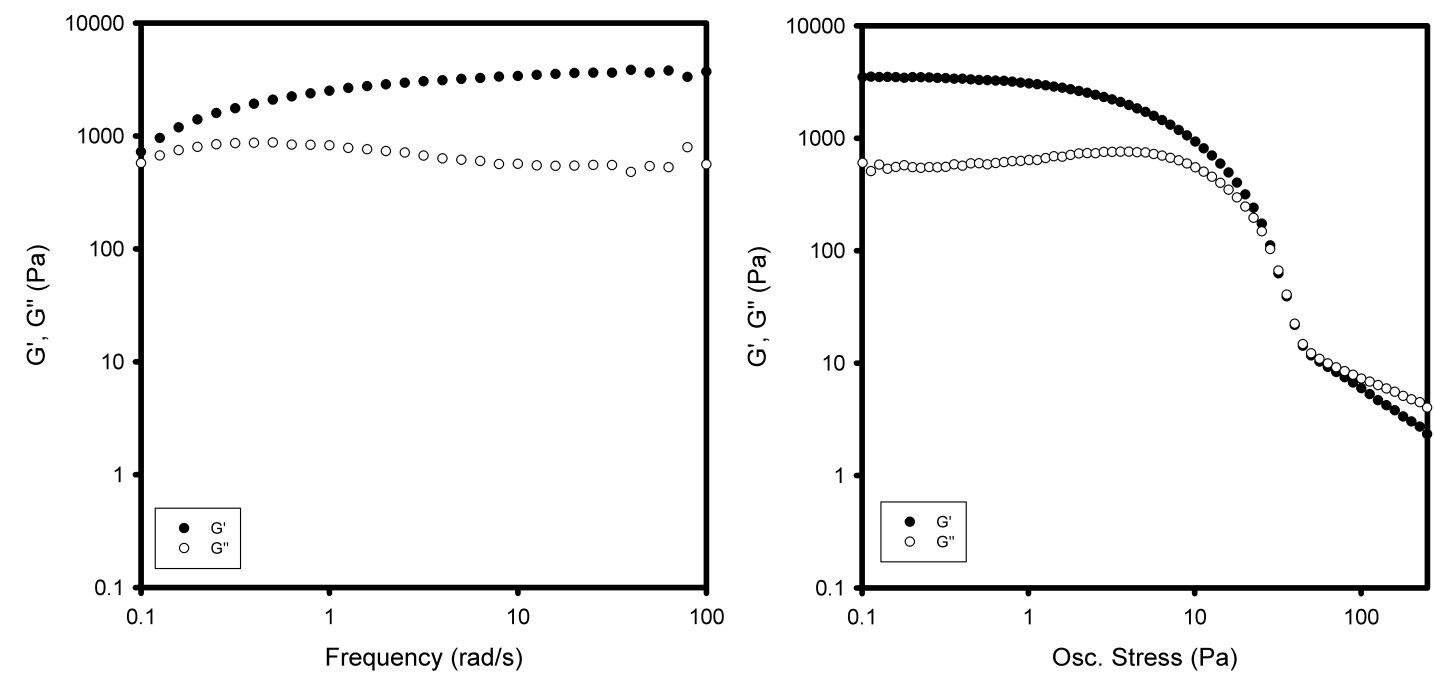

Figure S29. (A) Frequency sweep and (B) oscillating stress sweep of a gel of $1 \mathbf{d}(6.76 \mathrm{mM}$ in EtOAc); $400 \mu \mathrm{m}$. Storage modulus $\left(\mathrm{G}^{\prime}, \bullet\right)$ and loss modulus $\left(\mathrm{G}^{\prime \prime}, \circ\right)$. Analyzed after $\sim 18 \mathrm{~h}$.
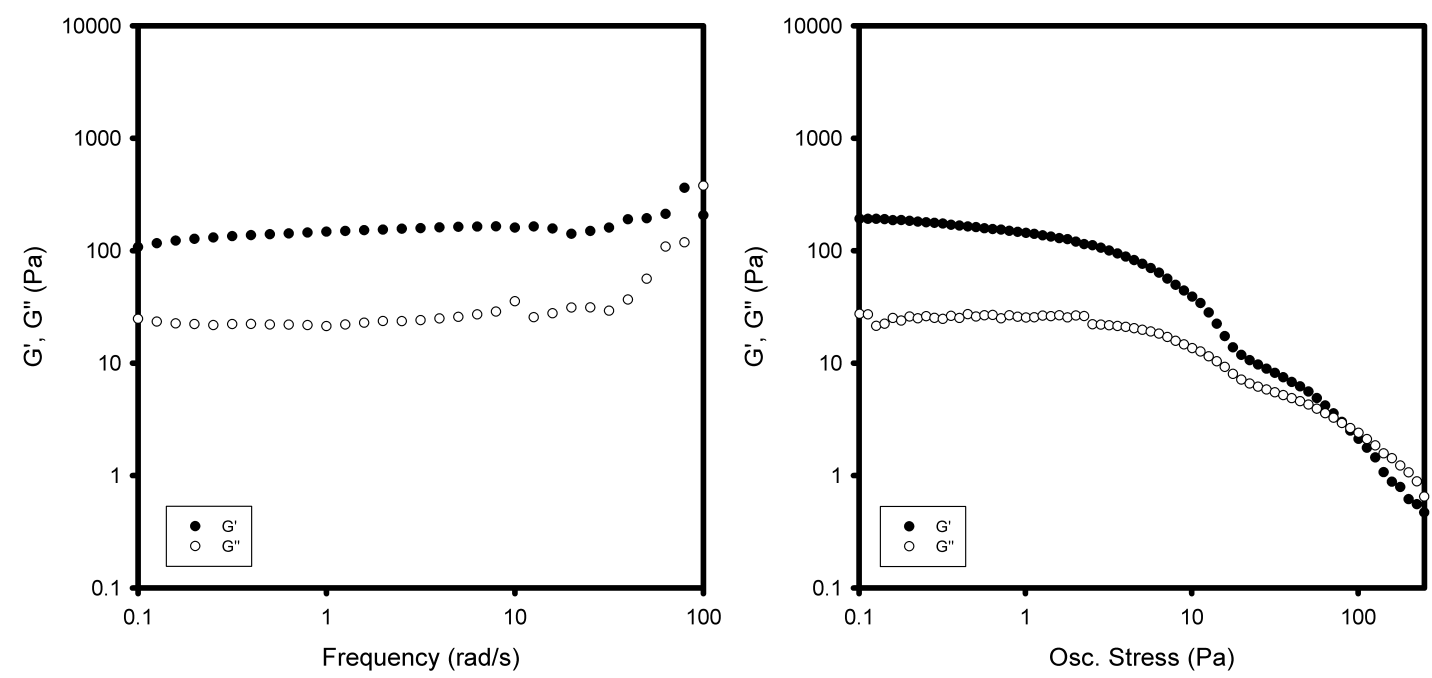

Figure S30. (A) Frequency sweep and (B) oscillating stress sweep of a gel of $\mathbf{1 d}(1.55 \mathrm{mM}$ in paint thinner); $300 \mu \mathrm{m}$ gap. Storage modulus $\left(\mathrm{G}^{\prime}, \bullet\right)$ and loss modulus $\left(\mathrm{G}^{\prime \prime}, \circ\right)$. Analyzed after 6 h. 

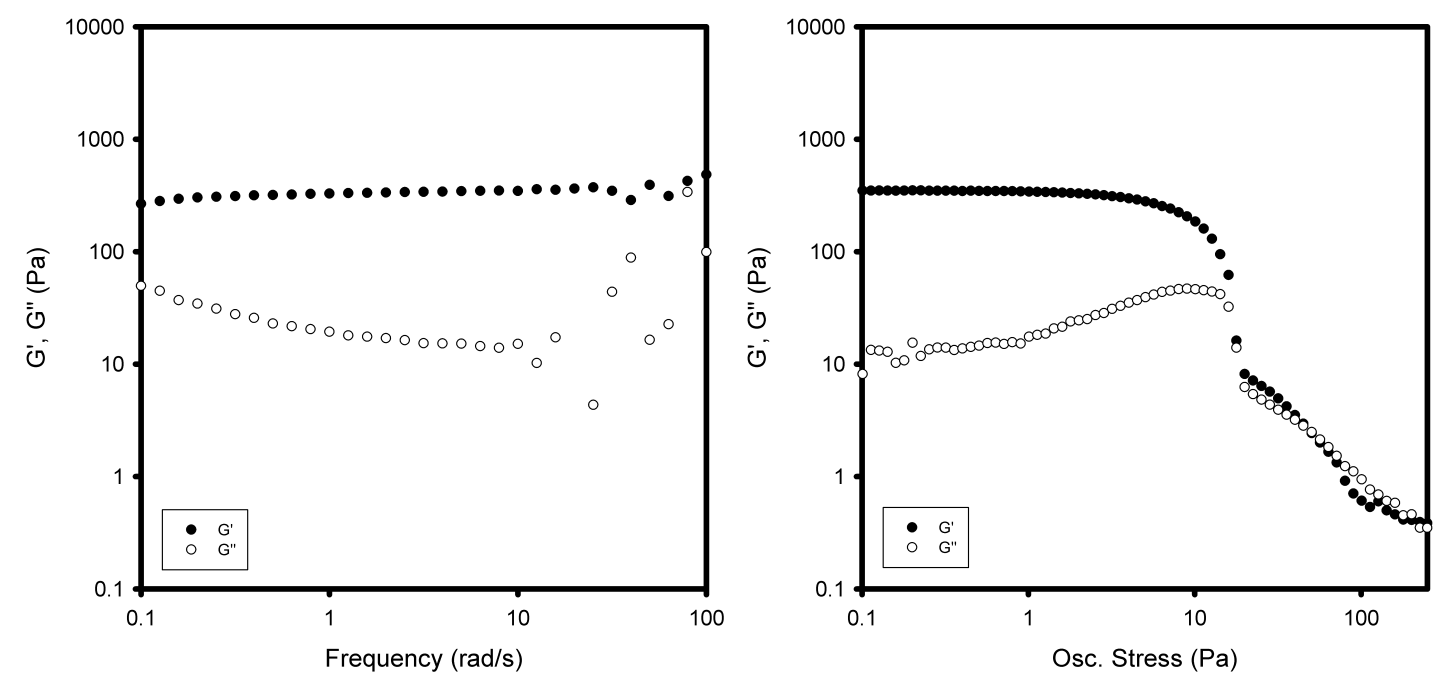

Figure S31. (A) Frequency sweep and (B) oscillating stress sweep of a gel of 1e $(6.42 \mathrm{mM}$ in paint thinner); $300 \mu \mathrm{m}$ gap. Storage modulus $\left(\mathrm{G}^{\prime}, \bullet\right)$ and loss modulus $\left(\mathrm{G}^{\prime \prime}, \circ\right)$.
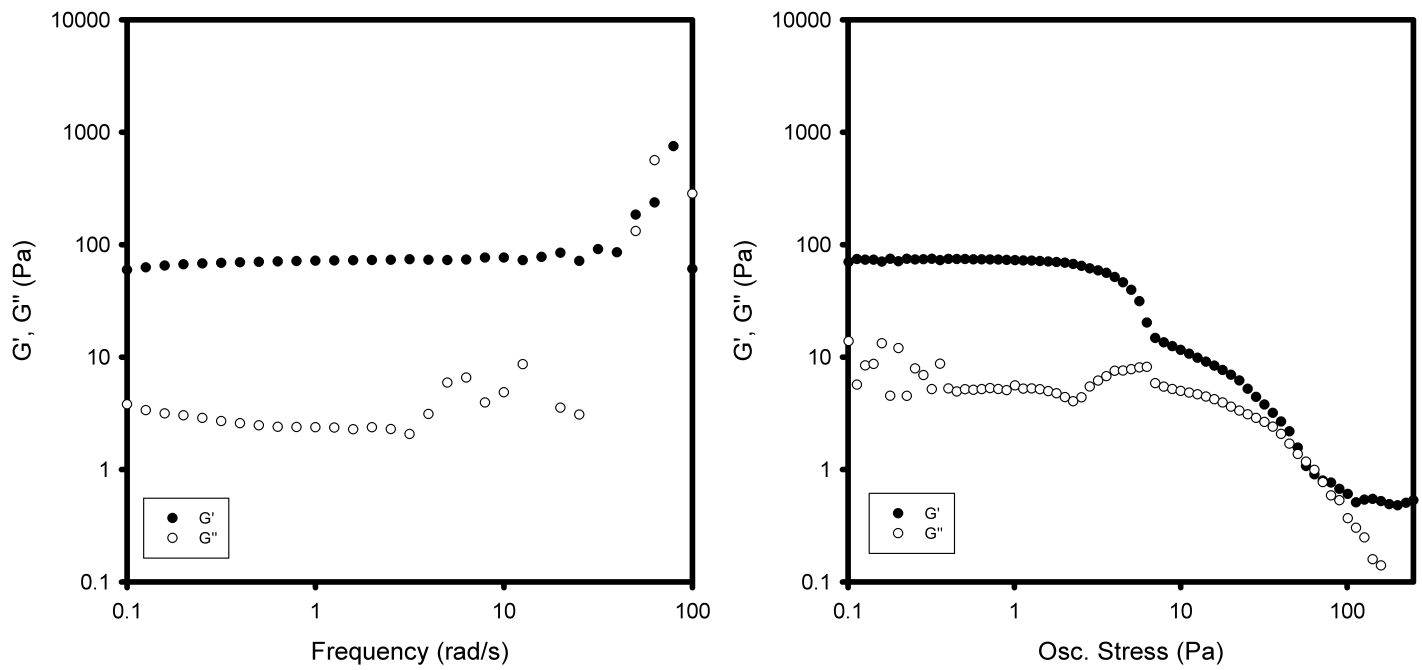

Figure S32. (A) Frequency sweep and (B) oscillating stress sweep of a gel of $1 \mathbf{f}$ (1.64 mM in paint thinner); $400 \mu \mathrm{m}$ gap. Storage modulus $\left(\mathrm{G}^{\prime}, \bullet\right)$ and loss modulus $\left(\mathrm{G}^{\prime \prime}, \circ\right)$. 

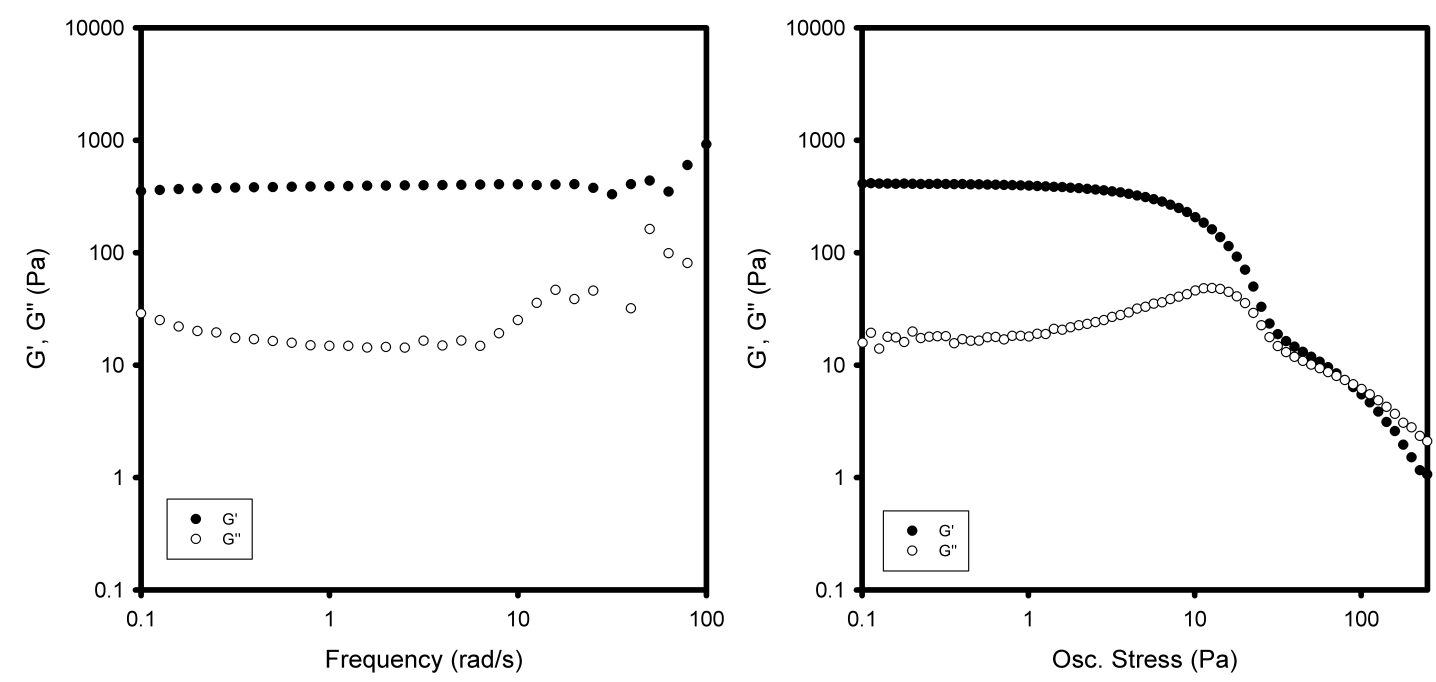

Figure S33. (A) Frequency sweep and (B) oscillating stress sweep of a gel of $\mathbf{1 g}(2.24 \mathrm{mM}$ in paint thinner); $500 \mu \mathrm{m}$ gap. Storage modulus $\left(\mathrm{G}^{\prime}, \bullet\right)$ and loss modulus $\left(\mathrm{G}^{\prime}, \circ\right)$.
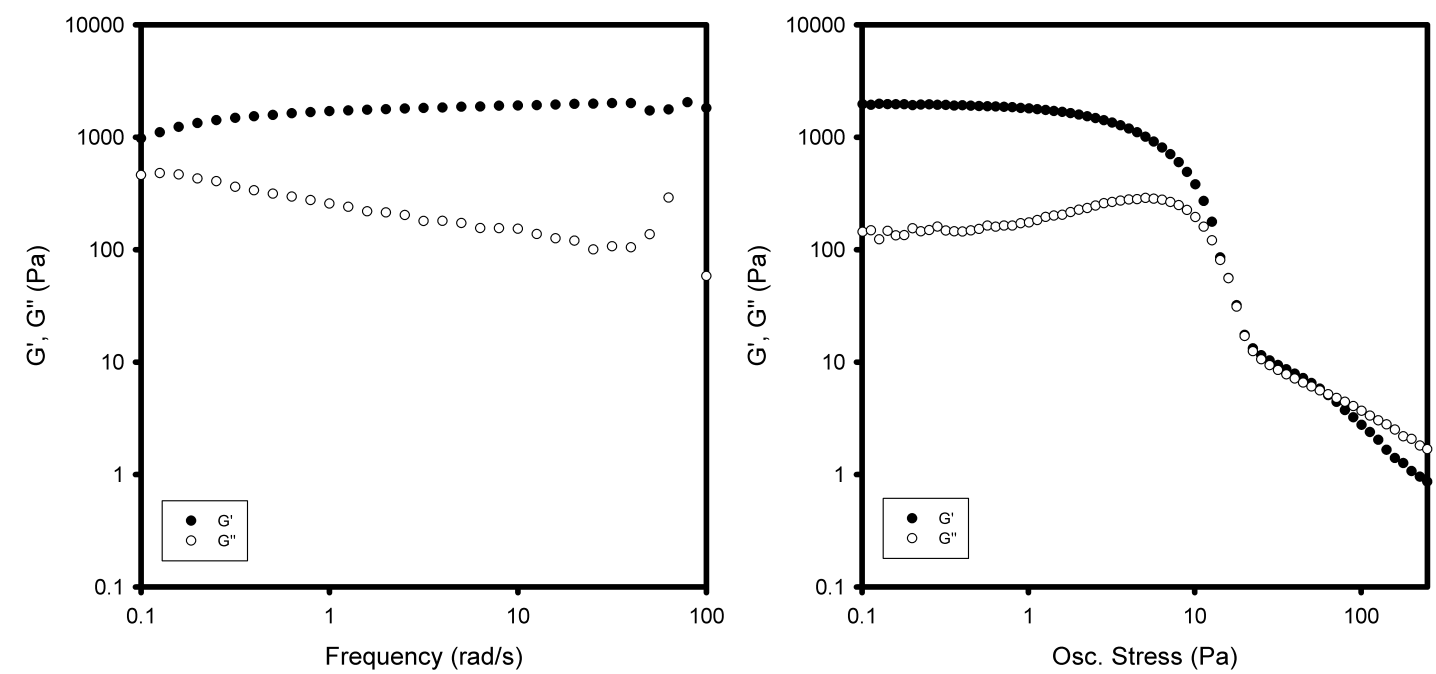

Figure S34. (A) Frequency sweep and (B) oscillating stress sweep of a gel of $1 \mathrm{~h}(5.76 \mathrm{mM}$ in paint thinner); $400 \mu \mathrm{m}$ gap. Storage modulus $\left(\mathrm{G}^{\prime}, \bullet\right)$ and loss modulus (G", ०). 

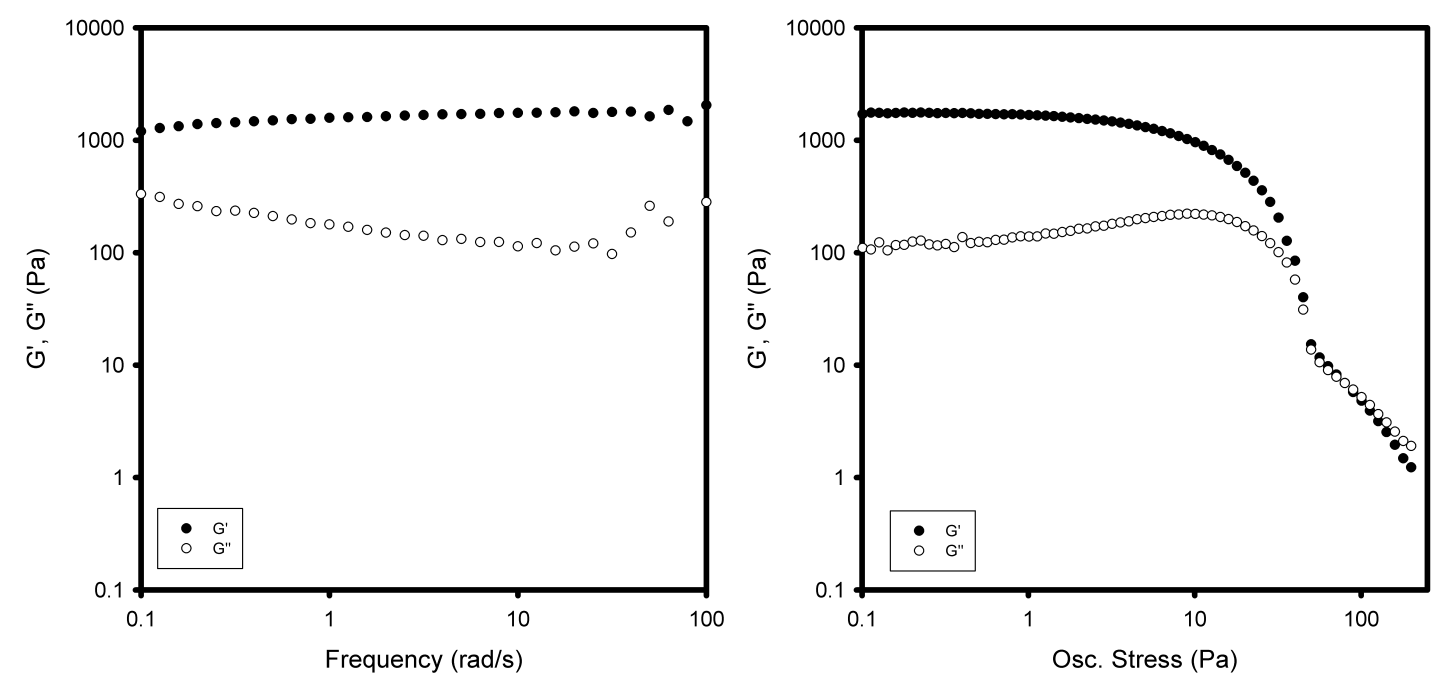

Figure S35. (A) Frequency sweep and (B) oscillating stress sweep of a gel of $\mathbf{2 b}(16.1 \mathrm{mM}$ in $\mathrm{H}_{2} \mathrm{O} / \mathrm{EtOH}$ solution at $\left.\mathrm{pH} 12\right) ; 500 \mu \mathrm{m}$ gap. Storage modulus $\left(\mathrm{G}^{\prime}, \bullet\right)$ and loss modulus $\left(\mathrm{G}^{\prime \prime}, \circ\right)$. 


\section{Gel Characterization via Scanning Electron Microscopy}

General Procedure - Wet gels of $\mathbf{1 d}-\mathbf{1 h}$ and $\mathbf{2 b}$ at concentrations of $\sim 1.5-2 \times$ cgc were mounted with copper tape onto a stainless steel SEM holder and the solvent was evaporated at rt over night or longer. Samples were sputter-coated with Au for $30 \mathrm{~s}$ to reduce charge build-up during imaging. All gels were imaged using the high vacuum mode on a FEI NOVA Nanolab Dualbeam Workstation with a Schottky field emitter operated at 10-20 kV accelerating voltage or a Hitachi S3200N SEM using a 15-kV accelerating voltage. The images were digitally recorded.

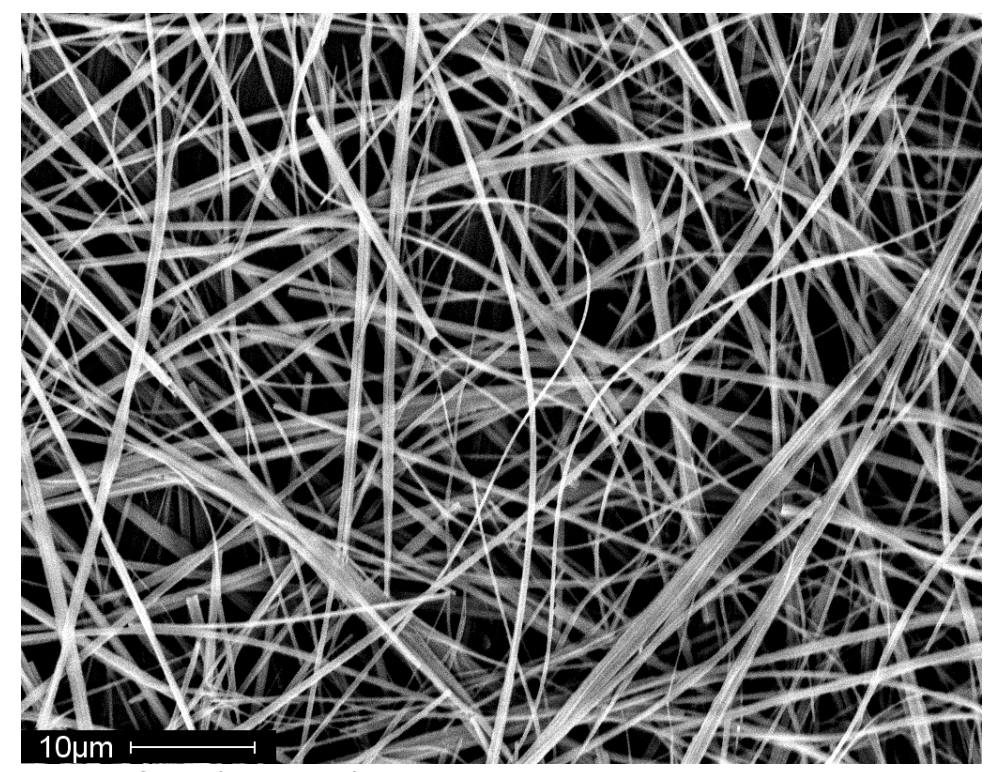

Figure S36. SEM image of $1 \mathbf{d}(2.7 \mathrm{mM})$ in acetone.

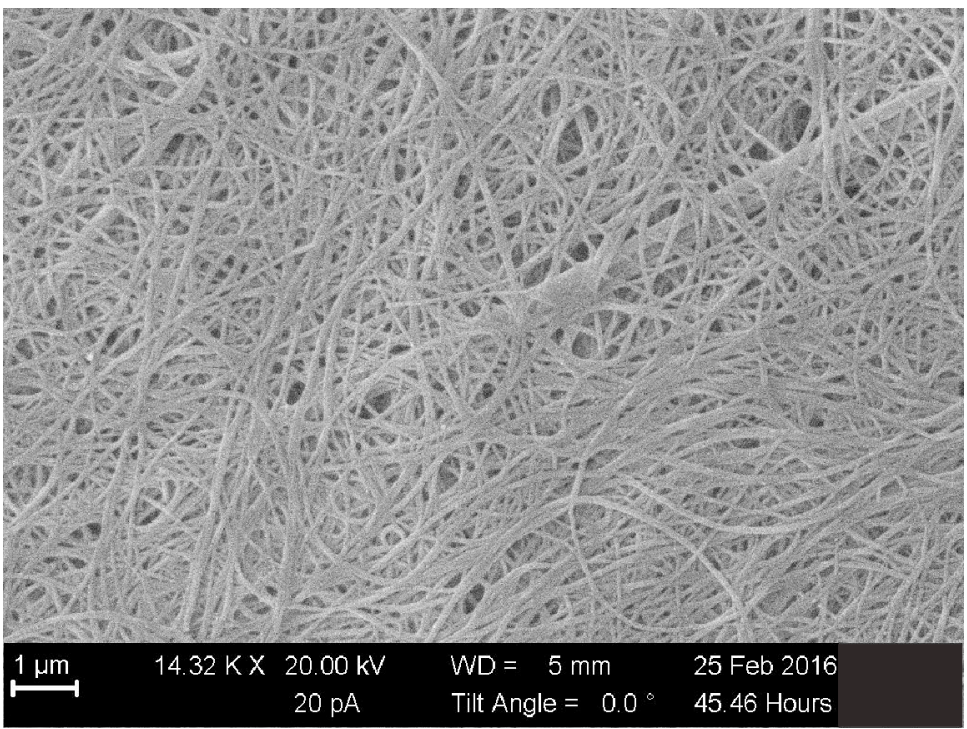

Figure S37. SEM image of $1 \mathbf{d}(1.56 \mathrm{mM})$ in paint thinner. 


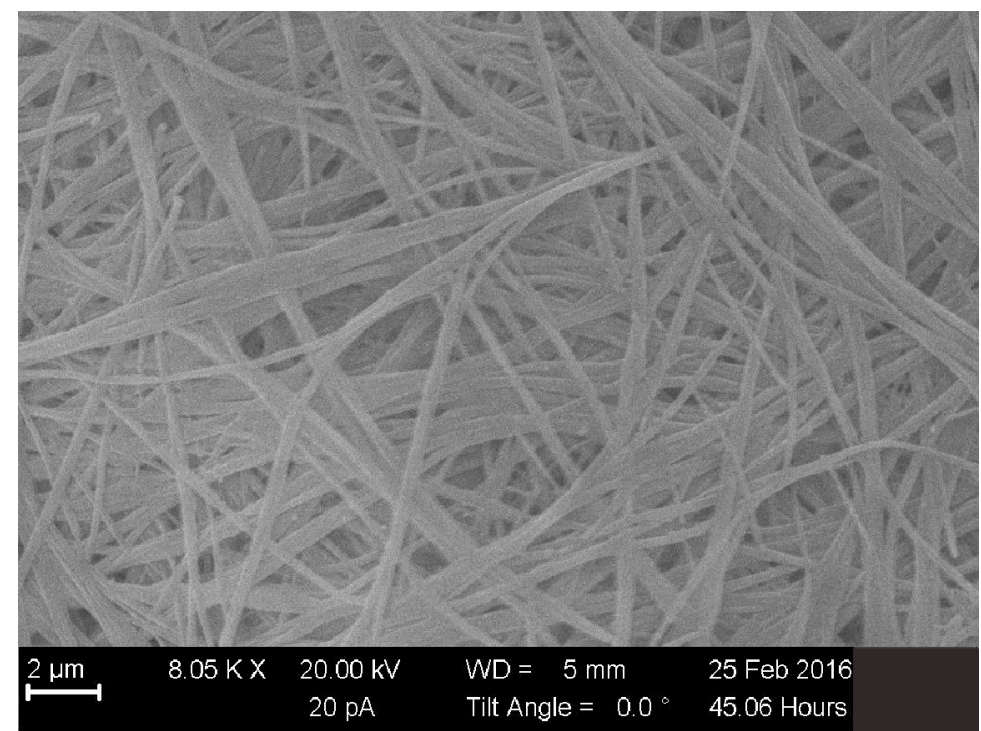

Figure S38. SEM image of 1e $(6.21 \mathrm{mM})$ in paint thinner.

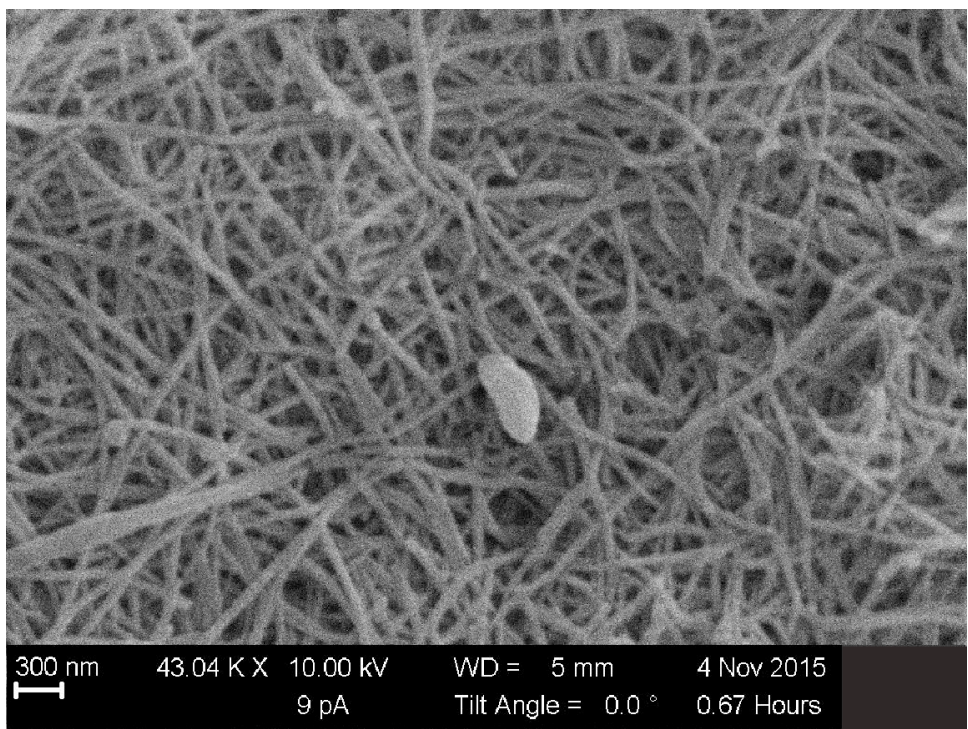

Figure S39. SEM image of $1 \mathrm{f}(1.91 \mathrm{mM})$ in paint thinner. 


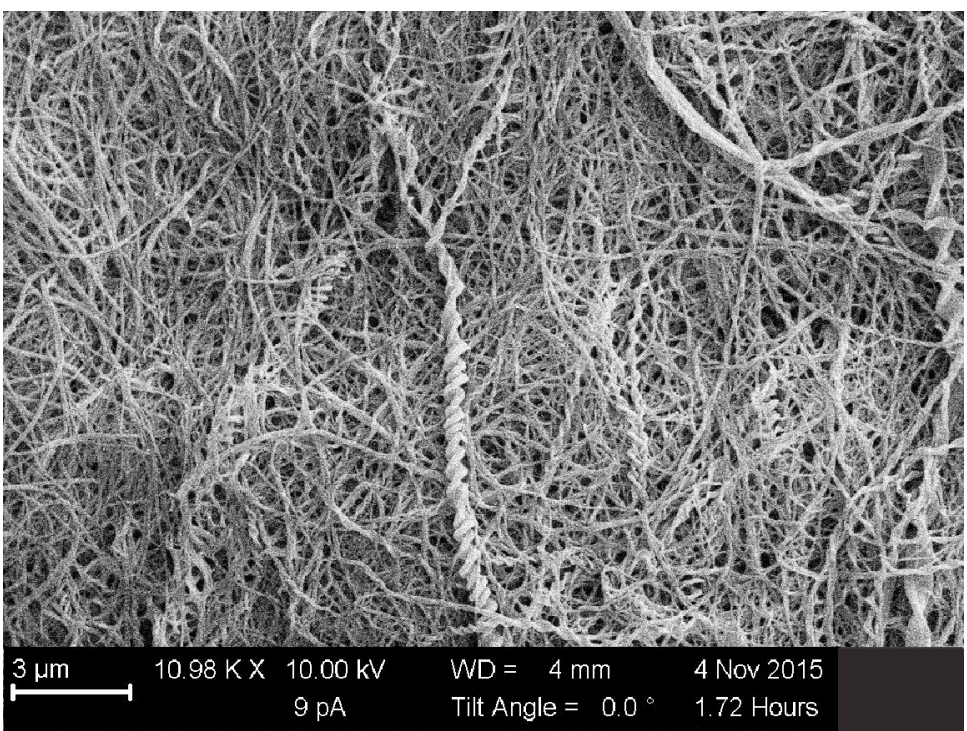

Figure S40. SEM image of $1 \mathrm{~g}(2.18 \mathrm{mM})$ in paint thinner.

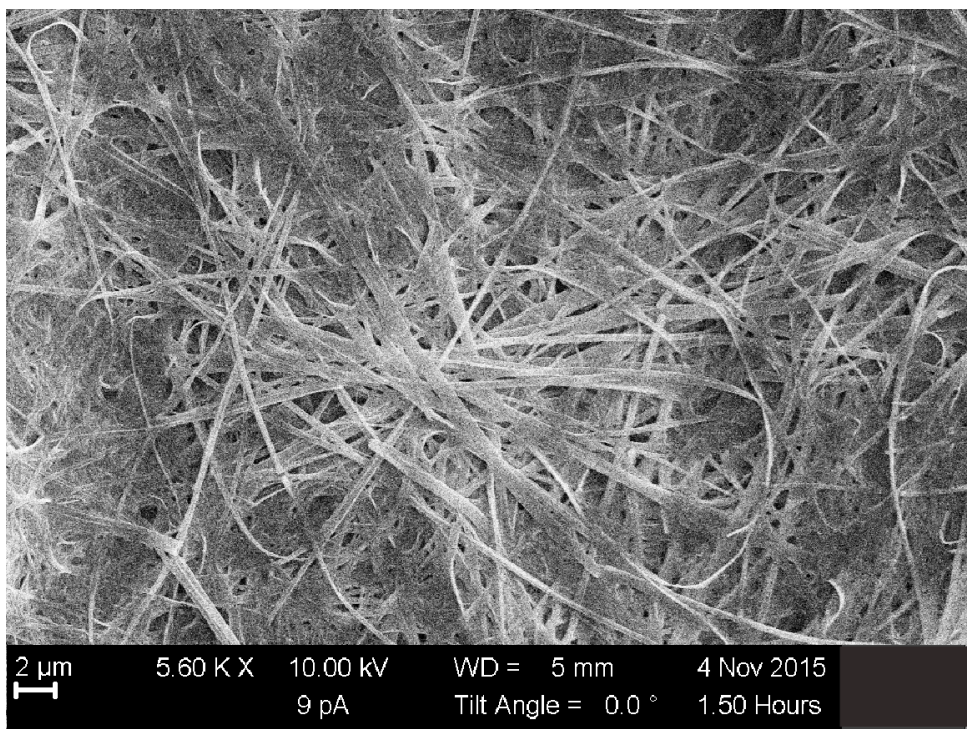

Figure S41. SEM image of $1 \mathrm{~h}(5.18 \mathrm{mM})$ in EtOAc. 


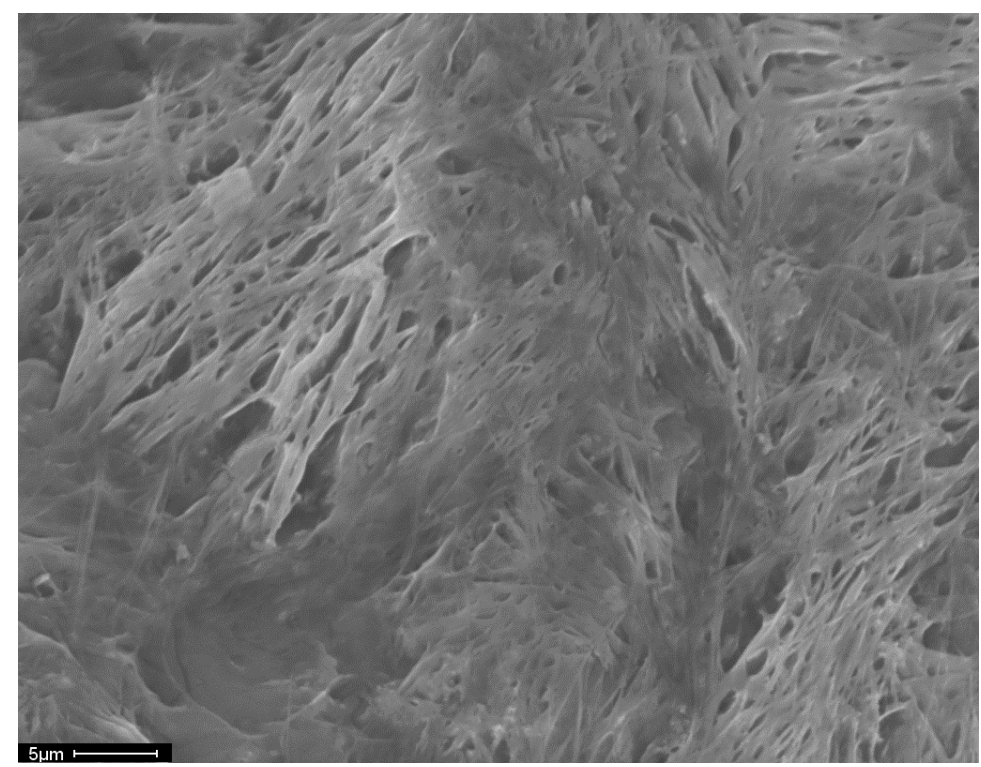

Figure S42. SEM image of $\mathbf{2 b}(13.9 \mathrm{mM})$ in basic $\mathrm{H}_{2} \mathrm{O} / \mathrm{EtOH}$ solution $(\mathrm{pH} 12)$. 


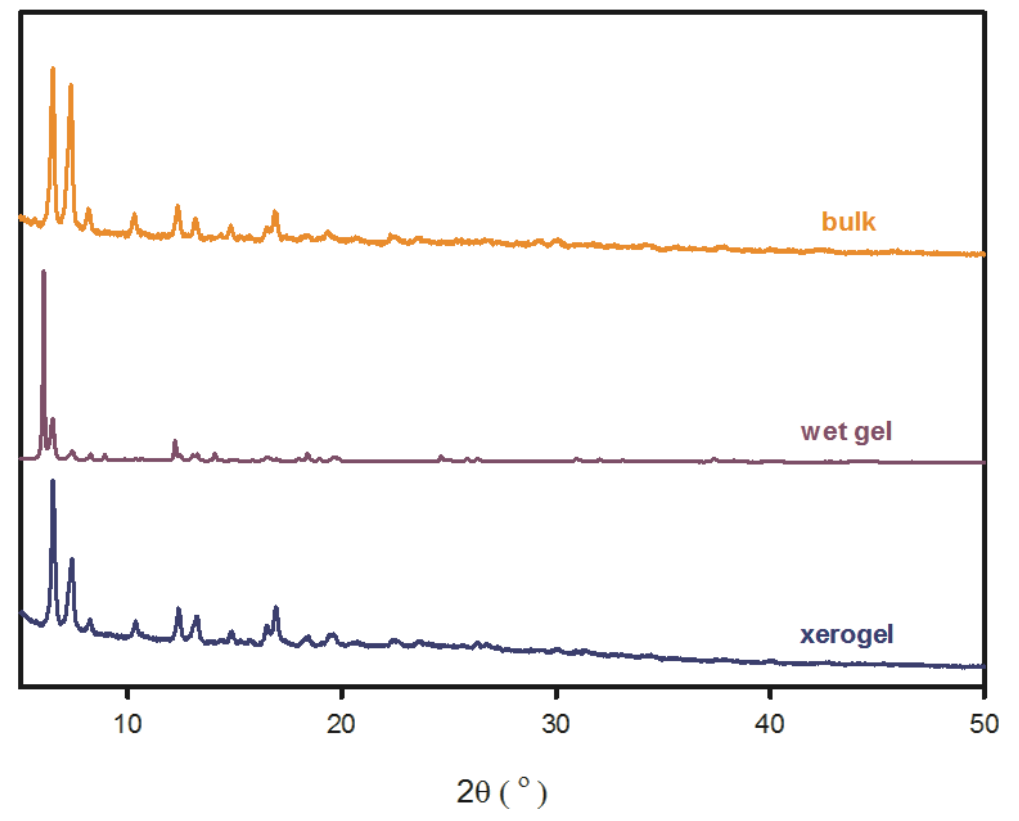

Figure S43. Normalized PXRD patterns of 1d: bulk from synthesis, wet gel (acetone), and xerogel (dry gel from acetone).

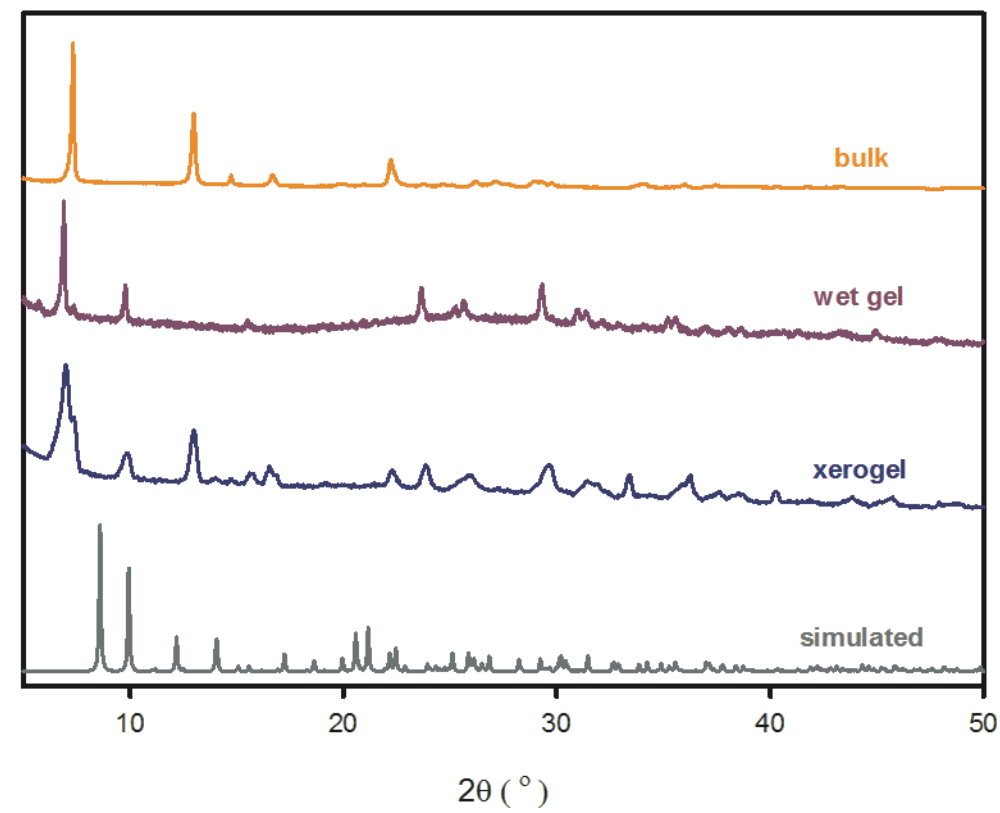

Figure S44. Normalized PXRD patterns of 2b: bulk from synthesis, wet gel (basic $\mathrm{H}_{2} \mathrm{O} / \mathrm{EtOH}$ solution, $\mathrm{pH}$ 12), xerogel (dry gel from basic $\mathrm{H}_{2} \mathrm{O} / \mathrm{EtOH}$ solution, $\mathrm{pH} 12$ ), and simulated PXRD pattern from crystal structure of $\mathbf{2 b}$. 


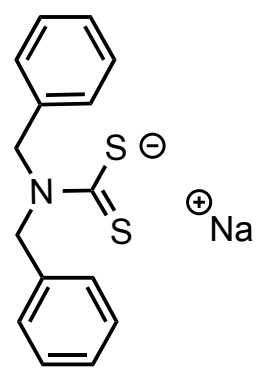

3

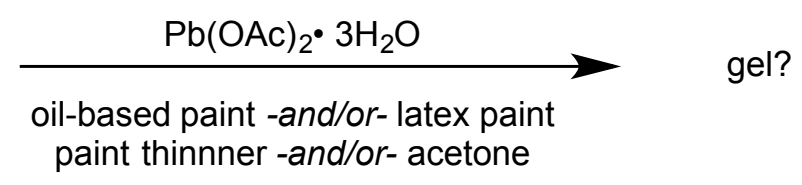

General Procedure - In an $8 \mathrm{~mL}$ vial, a known amount of $\mathrm{Pb}(\mathrm{OAc})_{2} \cdot 3 \mathrm{H}_{2} \mathrm{O}$ in $0.5 \mathrm{~mL}$ solvent was treated with a solution of 3 in $0.5 \mathrm{~mL}$ of solvent. The vial was capped and sonicated for several seconds to ensure complete mixing before $0.05 \mathrm{~mL}$ paint was added. The vial was heated and sonicated to mix the reagents. Once mixing appeared complete, the vial was heated to dissolve the solid, and subsequently cooled with approximately $20 \mathrm{~s}$ of sonication in an $\mathrm{H}_{2} \mathrm{O}$ bath (near ambient temperature). After standing undisturbed at $\mathrm{rt}$ for at least $10 \mathrm{~min}$, the vial was inverted to test for gelation.

\section{Paint used:}

black oil-based paint (soluble in paint thinner): Rust-Oleum Professional, V7579 Gloss Black, High Performance Protective Enamel

pink latex-based paint (soluble in acetone): Valspar Satin Berry Twist 530832, Spring 2014

Table S14. Testing gelation in presence of paint.

\begin{tabular}{cccccc}
\hline vial & $\begin{array}{c}\mathbf{3} \\
(\mathbf{m g})\end{array}$ & $\begin{array}{c}\mathbf{P b}(\mathbf{O A c})_{2} \bullet \mathbf{3 H}_{\mathbf{2}} \mathbf{O} \\
(\mathbf{m g})\end{array}$ & solvent & paint & gel? \\
\hline $\mathbf{1}$ & 4.2 & -- & paint thinner & black & no \\
$\mathbf{2}$ & 4.0 & 2.7 & paint thinner & -- & yes \\
$\mathbf{3}$ & 4.1 & 2.7 & paint thinner & black & yes \\
$\mathbf{4}$ & 3.9 & 2.5 & paint thinner:acetone & $\begin{array}{c}\text { black and } \\
\text { pink }^{\mathrm{b}}\end{array}$ & yes \\
$\mathbf{5}$ & 4.2 & 2.9 & acetone & pink & yes \\
$\mathbf{6}$ & 4.2 & 2.4 & acetone & -- & yes \\
$\mathbf{7}$ & 3.9 & -- & acetone & pink & no \\
\hline
\end{tabular}

a) $1 \mathrm{~mL}$ solvent. b) $0.025 \mathrm{~mL}$ of each paint. 


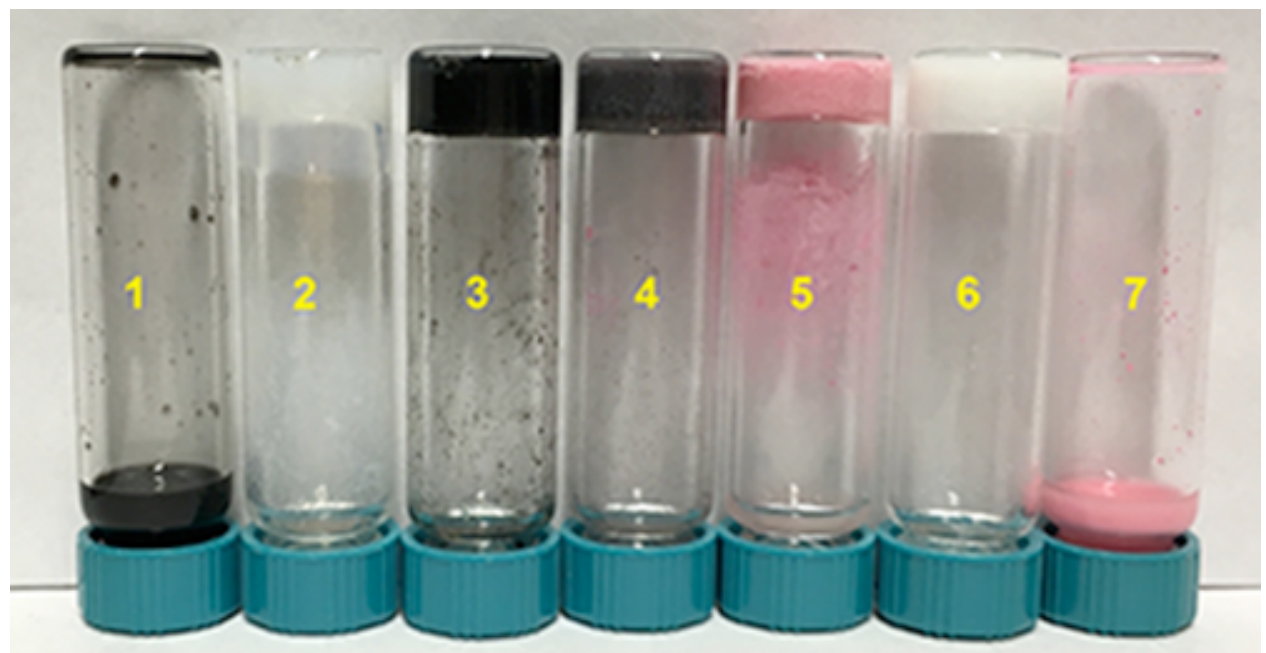

Figure S45. Image of gels and solutions testing for gelation in presence of oil-based (black) and/or latex-based (pink) paint in paint thinner and/or acetone. 
XVI. Gelation with Different Lead(II) Sources

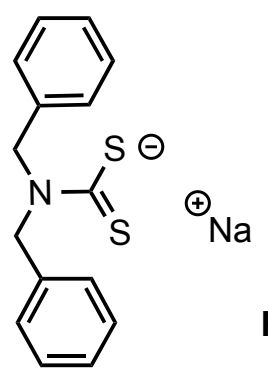

$\oplus_{\mathrm{Na}} \underset{\text { paint thinnner, } \mathrm{MeOH}}{\stackrel{\mathrm{Pb} \text { salt }}{\longrightarrow}}$
$\mathrm{Pb}$ salt $=\mathrm{PbO}, \mathrm{PbCrO}_{4}, \mathrm{~Pb}\left(\mathrm{NO}_{3}\right)_{2}, \mathrm{PbCO}_{3}$

3

General Procedure - A known amount of $\mathrm{Pb}$ salt $\left(\mathrm{PbO}, \mathrm{PbCrO}_{4}, \mathrm{~Pb}\left(\mathrm{NO}_{3}\right)_{2}\right.$, or $\left.\mathrm{PbCO}_{3}\right)$ in $1 \mathrm{~mL}$ paint thinner in an $8 \mathrm{~mL}$ vial, was treated with a solution of 3 in $0.2 \mathrm{~mL} \mathrm{MeOH}$. The vial was capped and sonicated for $20 \mathrm{~s}$ to ensure complete mixing. Once mixing appeared complete, the vial was heated to dissolve the solid, and subsequently cooled with approximately $20 \mathrm{~s}$ of sonication in an $\mathrm{H}_{2} \mathrm{O}$ bath (near ambient temperature). After standing undisturbed at rt for 10-15 min, the vial was inverted to test for gelation. The heating and sonication steps were repeated if no gel formed.

Table S15. Testing gelation with different lead(II) salts.

\begin{tabular}{ccccc|}
\hline $\mathrm{Pb}$ salt & mg Pb salt & $\mathbf{3}(\mathbf{m g})$ & gel? & $\begin{array}{c}\text { \# heating } \\
\text { cycles }\end{array}$ \\
\hline $\mathrm{PbO}$ & 2.2 & 4.2 & yes & 2 \\
\hline $\mathrm{PbCO}_{3}$ & 2.0 & 3.8 & yes & 1 \\
$\mathrm{PbCrO}_{4}$ & 2.0 & 4.2 & yes & 2 \\
\hline $\mathrm{Pb}\left(\mathrm{NO}_{3}\right)_{2}$ & 2.2 & 4.3 & no & $4^{\mathrm{a}}$ \\
\hline
\end{tabular}

a) After the third heating cycle, fiber formation was visible, but no stable gel formed.

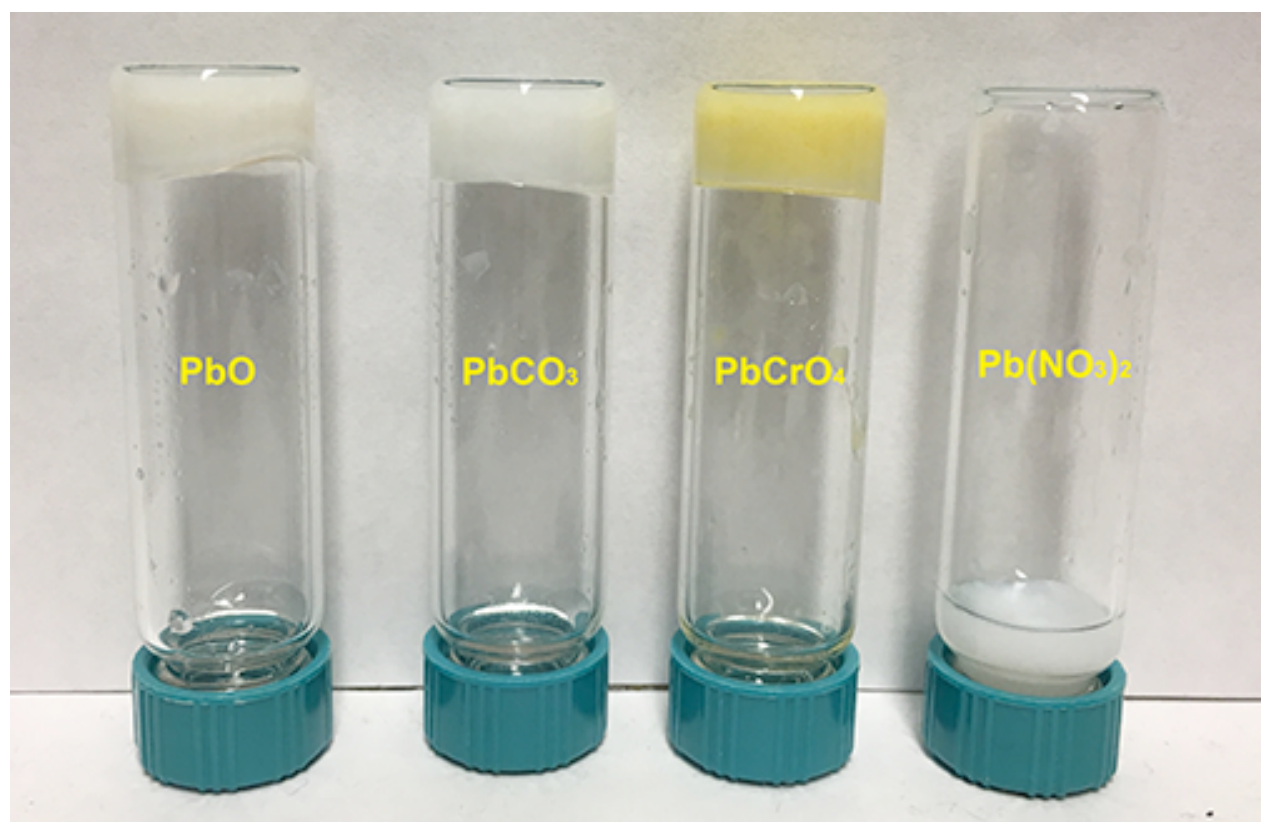

Figure S46. Image of gels and mixtures with different lead sources: $\mathrm{PbO}, \mathrm{PbCO}_{3}, \mathrm{PbCrO}_{4}$ and $\mathrm{Pb}\left(\mathrm{NO}_{3}\right)_{2}$. 

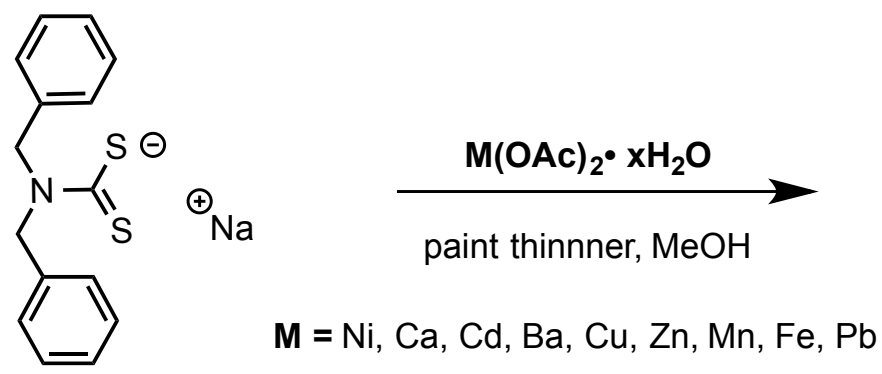

3

General Procedure - A known amount of metal acetate salt $\left(\mathrm{Ni}(\mathrm{OAc})_{2} \bullet 4 \mathrm{H}_{2} \mathrm{O}, \mathrm{Ca}(\mathrm{OAc})_{2}\right.$, $\mathrm{Cd}(\mathrm{OAc})_{2} \cdot 2 \mathrm{H}_{2} \mathrm{O}, \quad \mathrm{Ba}(\mathrm{OAc})_{2}, \quad \mathrm{Cu}(\mathrm{OAc})_{2} \cdot 2 \mathrm{H}_{2} \mathrm{O}, \quad \mathrm{Zn}(\mathrm{OAc})_{2} \cdot 2 \mathrm{H}_{2} \mathrm{O}, \quad \mathrm{Mn}(\mathrm{OAc})_{2}, \quad \mathrm{Fe}(\mathrm{OAc})_{2}, \quad$ or $\left.\mathrm{Pb}(\mathrm{OAc})_{2} \cdot 3 \mathrm{H}_{2} \mathrm{O}\right)$ in $1 \mathrm{~mL}$ paint thinner in an $8 \mathrm{~mL}$ vial, was treated with a solution of $4.1 \mathrm{mg} 3 \mathrm{in}$ $0.2 \mathrm{~mL} \mathrm{MeOH}$. The vial was capped and sonicated for $20 \mathrm{~s}$ to ensure complete mixing. Once mixing appeared complete, the vial was heated to dissolve the solid, and subsequently cooled with approximately $20 \mathrm{~s}$ of sonication in an $\mathrm{H}_{2} \mathrm{O}$ bath (near ambient temperature). After standing undisturbed at rt for $\sim 10-15 \mathrm{~min}$, the vial was inverted to test for gelation. The heating and sonication steps were repeated if no gel formed.

Table S16. Testing gelation with various metal acetate salts.

\begin{tabular}{|c|c|c|c|}
\hline $\mathrm{M}(\mathrm{OAC})_{2} \cdot \times \mathrm{H}_{2} \mathrm{O}$ & mg & gel? & appearance \\
\hline $\mathrm{Ni}(\mathrm{OAc})_{2} \bullet 4 \mathrm{H}_{2} \mathrm{O}$ & 1.7 & no & green ppt \\
\hline $\mathrm{Ca}(\mathrm{OAc})_{2}$ & 1.2 & no & white ppt \\
\hline $\mathrm{Cd}(\mathrm{OAc})_{2} \cdot 2 \mathrm{H}_{2} \mathrm{O}$ & 1.4 & no & clear solution \\
\hline $\mathrm{Ba}(\mathrm{OAc})_{2}$ & 1.7 & no & clear solution \\
\hline $\mathrm{Cu}(\mathrm{OAc})_{2} \cdot 2 \mathrm{H}_{2} \mathrm{O}$ & 1.4 & no & brown solution \\
\hline $\mathrm{Zn}(\mathrm{OAc})_{2} \cdot 2 \mathrm{H}_{2} \mathrm{O}$ & 1.9 & no & clear solution \\
\hline$M n(O A c)_{2}$ & 2.2 & no & brown/purple ppt \\
\hline $\mathrm{Fe}(\mathrm{OAc})_{2}$ & 1.1 & no & brown/red ppt \\
\hline $\mathrm{Pb}(\mathrm{OAc})_{2} \cdot 3 \mathrm{H}_{2} \mathrm{O}$ & 2.7 & yes & white gel \\
\hline
\end{tabular}

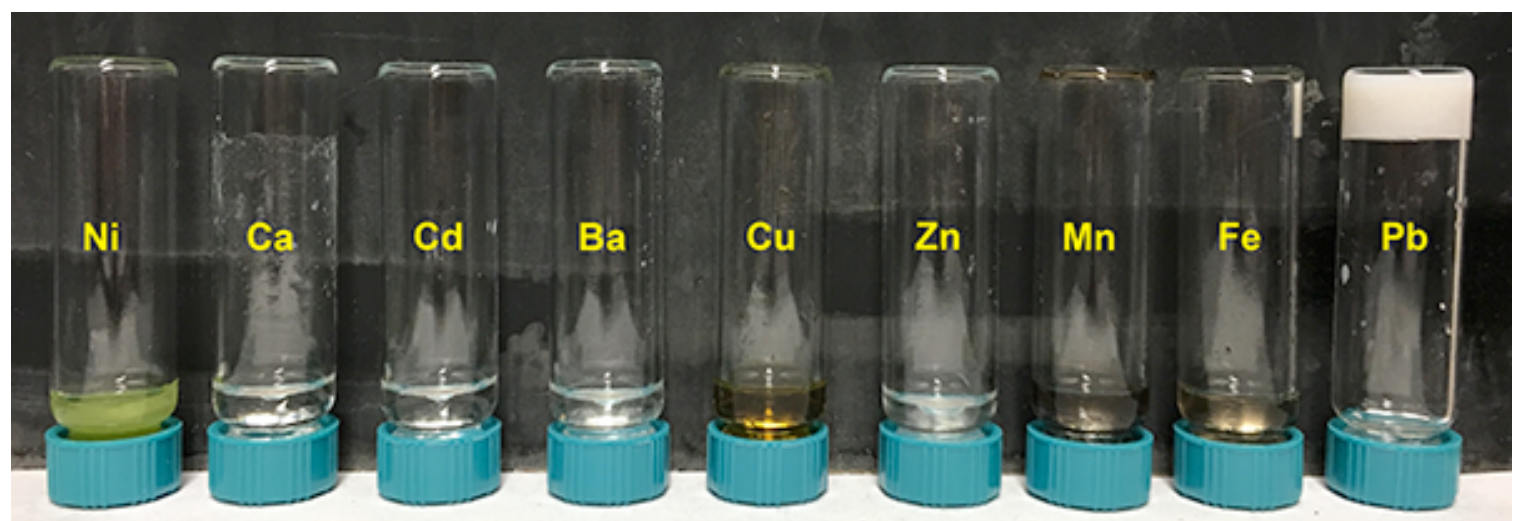

Figure S47. Image of $\mathbf{3}$ with different metal acetate salts ( $\mathrm{Ni}, \mathrm{Ca}, \mathrm{Cd}, \mathrm{Ba}, \mathrm{Cu}, \mathrm{Zn}, \mathrm{Mn}, \mathrm{Fe}$, and $\mathrm{Pb}$ ). 
XVIII. Testing Gelation with Lead(II) in the Presence of Other Metal Salts
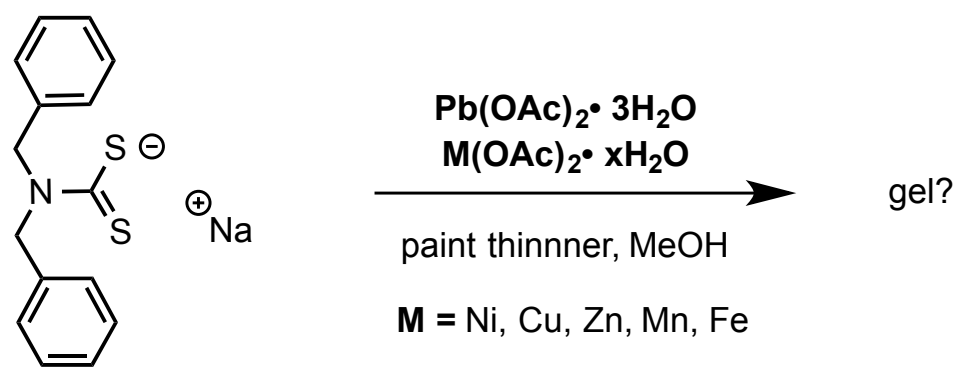

3

General Procedure - A solution of $\mathrm{Pb}(\mathrm{OAc})_{2} \cdot 3 \mathrm{H}_{2} \mathrm{O}(2.7 \mathrm{mg}$ in $0.1 \mathrm{~mL} \mathrm{MeOH})$ was added to an 8 $\mathrm{mL}$ vial containing the other $\mathrm{M}(\mathrm{OAc})_{2} \bullet \mathrm{H}_{2} \mathrm{O}\left(\mathrm{Ni}(\mathrm{OAc})_{2} \cdot 4 \mathrm{H}_{2} \mathrm{O}, \mathrm{Cu}(\mathrm{OAc})_{2} \cdot 2 \mathrm{H}_{2} \mathrm{O}, \mathrm{Zn}(\mathrm{OAc})_{2} \cdot 2 \mathrm{H}_{2} \mathrm{O}\right.$, $\mathrm{Mn}(\mathrm{OAc})_{2}$, or $\left.\mathrm{Fe}(\mathrm{OAc})_{2}\right)$ in $1 \mathrm{~mL}$ paint thinner. Subsequently $3(4.1 \mathrm{mg}$ in $0.1 \mathrm{~mL} \mathrm{MeOH})$ was added. The vial was capped and sonicated for $20 \mathrm{~s}$ to ensure complete mixing. Once mixing appeared complete, the vial was heated to dissolve the solid, and subsequently cooled with approximately $20 \mathrm{~s}$ of sonication in an $\mathrm{H}_{2} \mathrm{O}$ bath (near ambient temperature). After standing undisturbed at rt for $\sim 10-15 \mathrm{~min}$, the vial was inverted to test for gelation. The heating and sonication steps were repeated if no gel formed/no reaction occurred.

Table S17. Testing gelation with lead in the presence of other metal salts.

\begin{tabular}{cccc}
\hline $\mathrm{M}(\mathrm{OAc})_{2}{ }^{\bullet} \mathbf{x H}_{2} \mathrm{O}^{\mathrm{a}}$ & $\mathrm{mg}$ & gel? & appearance \\
\hline-- & -- & yes & white gel \\
$\mathrm{Ni}(\mathrm{OAc})_{2} \cdot \mathbf{4} \mathrm{H}_{2} \mathrm{O}$ & 1.7 & yes $^{\mathrm{b}}$ & green gel \\
$\mathrm{Cu}(\mathrm{OAc})_{2} \cdot 2 \mathrm{H}_{2} \mathrm{O}$ & 1.3 & yes $^{\mathrm{c}}$ & brown/white gel \\
$\mathrm{Zn}(\mathrm{OAc})_{2} \cdot 2 \mathrm{H}_{2} \mathrm{O}$ & 1.6 & yes & white gel \\
$\mathrm{Mn}(\mathrm{OAc})_{2}$ & 1.7 & yes & $\begin{array}{c}\text { white gel with } \\
\text { red/purple specs }\end{array}$ \\
$\mathrm{Fe}(\mathrm{OAc})_{2}$ & 1.2 & yes & yellow gel \\
\hline
\end{tabular}

a) All vials contain $\mathrm{Pb}(\mathrm{OAc})_{2} \cdot 3 \mathrm{H}_{2} \mathrm{O}$. b) An additional $2 \mathrm{mg} 3$ in $0.05 \mathrm{~mL} \mathrm{MeOH}$ was added to form a stable gel. c) An additional $5.1 \mathrm{mg} 3$ in $0.1 \mathrm{~mL} \mathrm{MeOH}$ was added to form a stable gel.

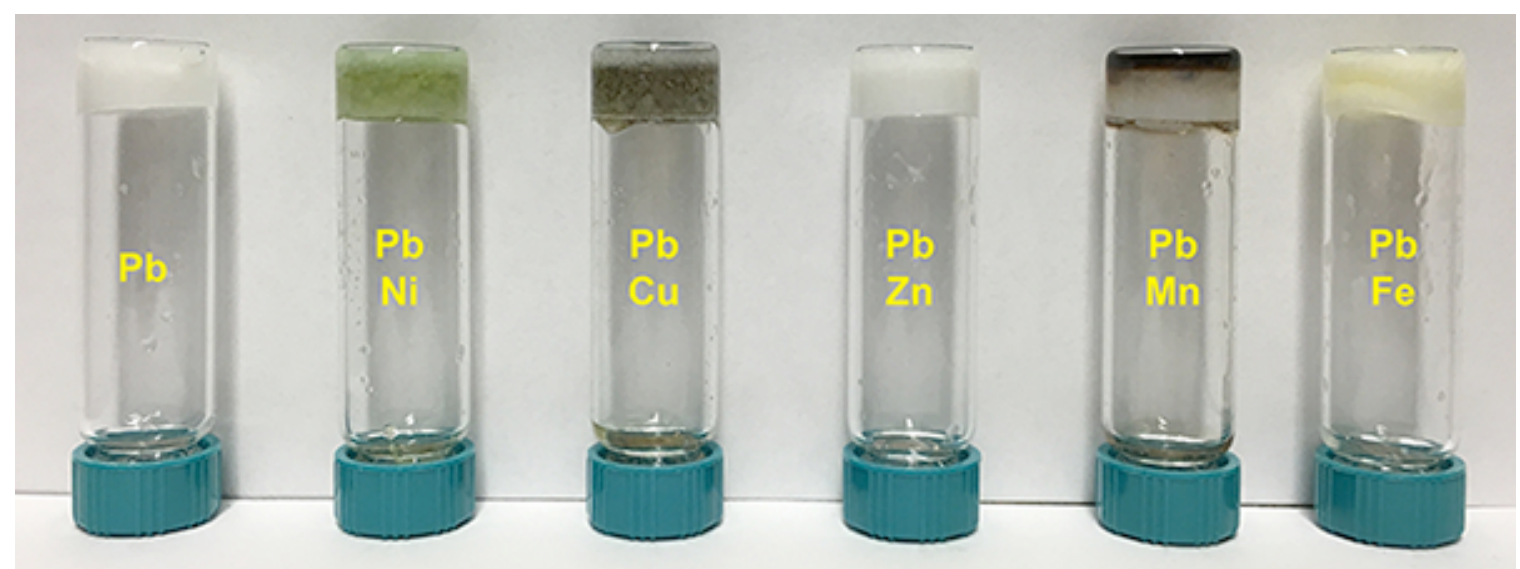

Figure S48. Image of formation of gels of $\mathbf{1 d}$ from reaction of $\mathbf{3}$ with a mixture of $\mathrm{Pb}(\mathrm{OAc})_{2} \cdot 3 \mathrm{H}_{2} \mathrm{O}$ in the presence of different metal acetate salts $(\mathrm{Ni}, \mathrm{Cu}, \mathrm{Zn}, \mathrm{Mn}$, and $\mathrm{Fe})$. 


\section{Developing a Sensor for 5000 ppm Pb in wet paint}

\section{A. Initial screening with black oil-based paint}

Determining the cgc for in situ formation of 1 d. A stock mixture of $5000 \mathrm{ppm}(5000 \mathrm{ppm}=$ $0.05 \%$ by weight $\mathrm{Pb}=6.6 \mathrm{mg} \mathrm{PbCO}_{3}$ in $998.4 \mathrm{mg}$ black oil-based paint) lead paint was prepared in $7.7 \mathrm{~mL}$ paint thinner. The lead-paint/paint thinner mixture was mixed well by sonicating for $\sim 60 \mathrm{~s}$ and vigorous shaking. Aliquots from this stock mixture are taken immediately after mixing. Since $\mathrm{PbCO}_{3}$ is insoluble, it is important to mix the stock mixture well prior to taking aliquots each time. An aliquot of $0.77 \mathrm{~mL}$ lead-paint mixture was taken and diluted with additional paint thinner $(0-0.23 \mathrm{~mL})$. Subsequently, $\sim 1.5 \mathrm{mg} 3$ was added as a solution in $\mathrm{MeOH}$ such that the total concentration of $\mathrm{MeOH}$ remains $\sim 17 \%$ by volume relative to the total volume of paint thinner.

General Procedure - To an $8 \mathrm{~mL}$ vial, $0.77 \mathrm{~mL}$ of the $5000 \mathrm{ppm}$ lead paint solution in paint thinner was added. If applicable, the solution was diluted with additional paint thinner. Subsequently, 3 is added as a solution in $\mathrm{MeOH}$. If applicable, additional $\mathrm{MeOH}$ was added prior to addition of the ligand solution. The vial was capped and sonicated for $20 \mathrm{~s}$ to ensure complete mixing. Once mixing appeared complete, the vial was heated to dissolve the solid, and subsequently cooled with approximately $20 \mathrm{~s}$ of sonication in an $\mathrm{H}_{2} \mathrm{O}$ bath (near ambient temperature). After standing undisturbed at $\mathrm{rt}$ for $\sim 10 \mathrm{~min}$, the vial was inverted to test for gelation.

Table S18. Determining the cgc for in situ gel formation in the presence of paint. ${ }^{a, b}$

\begin{tabular}{cccc}
\hline $\begin{array}{c}\text { total volume } \\
(\mathbf{m L})\end{array}$ & $\begin{array}{c}\text { additional paint } \\
\text { thinner }(\mathbf{m L})\end{array}$ & $\begin{array}{c}\text { volume } \\
\text { MeOH } \\
(\mathbf{m L})\end{array}$ & gel? \\
\hline 0.93 & 0 & 0.16 & yes \\
1.2 & 0.23 & 0.2 & no \\
1.1 & 0.15 & 0.18 & $\begin{array}{c}\text { almost } \\
\text { gel }\end{array}$ \\
$\mathbf{1 . 0}^{\mathrm{c}}$ & $\mathbf{0 . 0 6}$ & $\mathbf{0 . 1 7}$ & yes \\
\hline
\end{tabular}

a) For each sample, used $0.77 \mathrm{~mL}$ of a $6.6 \mathrm{mg} \mathrm{PbCO}_{3}$ in $998.4 \mathrm{mg}$ paint solution in $7.7 \mathrm{~mL}$ paint thinner. b) $0.16 \mathrm{~mL}$ of a solution containing $19.1 \mathrm{mg} 3$ in $2 \mathrm{~mL} \mathrm{MeOH}$ was used. Additional $\mathrm{MeOH}$ was added to sample vials to get to $17 \%$ by volume $\mathrm{MeOH}$. c) This experiment was repeated three times. A gel formed each time.

Sensing lead in paint at $3000,4000,5000$, and $6000 \mathrm{ppm}$. Using the cgc determined for in situ formation of $\mathbf{1 d}$ from $\mathbf{3}$ in paint thinner/MeOH using wet paint. The total volume of each sample was kept at $1 \mathrm{~mL}(0.83 \mathrm{~mL} \mathrm{~Pb}$ paint solution in paint thinner, $0.17 \mathrm{~mL}$ MeOH solution). The amount of paint is kept relatively constant for each sample since the cgc will be affected by the amount of paint present.

5000 ppm: $6.7 \mathrm{mg} \mathrm{PbCO}_{3}$ in $995.6 \mathrm{mg}$ black oil-based paint in $8.3 \mathrm{~mL}$ paint thinner. 6000 ppm: $7.6 \mathrm{mg} \mathrm{PbCO}_{3}$ in $995.6 \mathrm{mg}$ black oil-based paint in $8.3 \mathrm{~mL}$ paint thinner. 10,000 ppm: $13.1 \mathrm{mg} \mathrm{PbCO}_{3}$ in $994.0 \mathrm{mg}$ black oil-based paint in $8.3 \mathrm{~mL}$ paint thinner. 
0 ppm: $996.7 \mathrm{mg}$ black oil-based paint in $8.3 \mathrm{~mL}$ paint thinner.

To prepare 4000 and $3000 \mathrm{ppm}$ solutions, the appropriate amount of solution from the 5000 ppm or $6000 \mathrm{ppm}$ mixture was taken and diluted to $0.83 \mathrm{~mL}$ using the $0 \mathrm{ppm}$ paint solution. This insures that all samples contain the same amount of paint.

MeOH solution of 3: $19.1 \mathrm{mg} 3$ in $2.13 \mathrm{~mL} \mathrm{MeOH}$.

\section{Sensing at $\mathbf{5 0 0 0}$ ppm:}

General Procedure - To an $8 \mathrm{~mL}$ vial, $0.83 \mathrm{~mL}$ of the $5000 \mathrm{ppm}$ lead paint solution in paint thinner was added. Subsequently, $0.17 \mathrm{~mL}$ of a $19.1 \mathrm{mg}$ solution of 3 in $2.13 \mathrm{~mL} \mathrm{MeOH}$ was added. The vial was capped and sonicated for $20 \mathrm{~s}$ to ensure complete mixing. Subsequently, the vial was heated for $\sim 30-40 \mathrm{~s}$ to dissolve the solid, and then cooled with approximately $20 \mathrm{~s}$ of sonication in an $\mathrm{H}_{2} \mathrm{O}$ bath (near ambient temperature). After standing undisturbed at $\mathrm{rt}$ for $\sim 10$ min, the vial was inverted to test for gelation for the first try. If no gel formed, the vial was heated again followed by sonication and resting undisturbed at rt before inverting to test for gelation for the second try.

Table S19. Sensing at $5000 \mathrm{ppm}$.

\begin{tabular}{ccc}
\hline Trial & gel $^{\text {st }}$ try? $^{?}$ & $\begin{array}{c}\text { gel } 2^{\text {nd }} \\
\text { try? }\end{array}$ \\
\hline 1 & almost & yes \\
2 & no & yes \\
3 & yes & -- \\
4 & no & yes \\
5 & no & yes \\
6 & no & yes \\
$7^{a}$ & almost $^{b}$ & yes \\
$8^{a}$ & almost $^{b}$ & yes \\
$9^{a}$ & almost $^{b}$ & yes \\
$10^{a}$ & almost $^{b}$ & yes \\
\hline
\end{tabular}

a) The sample was prepared using $0.69 \mathrm{~mL}$ of the 6000 ppm solution diluted with $0.14 \mathrm{~mL} 0 \mathrm{ppm}$ paint solution. b) "almost" means that a gel-like mixture formed but did not stick to the bottom of the vial for more than 3 seconds upon inversion.

\section{Sensing at 6000 ppm:}

General Procedure - To an $8 \mathrm{~mL}$ vial, $0.83 \mathrm{~mL}$ of the $6000 \mathrm{ppm}$ lead paint solution in paint thinner was added. Subsequently, $0.17 \mathrm{~mL}$ of a $19.1 \mathrm{mg}$ solution of 3 in $2.13 \mathrm{~mL} \mathrm{MeOH}$ was added. The vial was capped and sonicated for $20 \mathrm{~s}$ to ensure complete mixing. Subsequently, the vial was heated for $\sim 30-40 \mathrm{~s}$ to dissolve the solid, and then cooled with approximately $20 \mathrm{~s}$ of sonication in an $\mathrm{H}_{2} \mathrm{O}$ bath (near ambient temperature). After standing undisturbed at $\mathrm{rt}$ for $\sim 10$ min, the vial was inverted to test for gelation for the first try. If no gel formed, the vial was heated again followed by sonication and resting undisturbed at rt before inverting to test for gelation for the second try.

Table S20. Sensing at $6000 \mathrm{ppm}$. 


\begin{tabular}{ccc}
\hline Trial & gel $\mathbf{1}^{\text {st }}$ try? & $\begin{array}{c}\text { gel } \mathbf{2}^{\text {nd }} \\
\text { try? }\end{array}$ \\
\hline 1 & no & yes \\
2 & no & yes \\
3 & yes & -- \\
4 & no & yes \\
5 & no & almost $^{\text {a }}$ \\
\hline
\end{tabular}

a) "almost" means that a gel-like mixture formed but did not stick to the bottom of the vial for more than 3 seconds upon inversion.

\section{Sensing at 10,000 ppm:}

General Procedure - To an $8 \mathrm{~mL}$ vial, $0.83 \mathrm{~mL}$ of the $10,000 \mathrm{ppm}$ lead paint solution in paint thinner was added. Subsequently, $0.17 \mathrm{~mL}$ of a $19.1 \mathrm{mg}$ solution of 3 in $2.13 \mathrm{~mL} \mathrm{MeOH}$ was added. The vial was capped and sonicated for $20 \mathrm{~s}$ to ensure complete mixing. Subsequently, the vial was heated for $\sim 30-40 \mathrm{~s}$ to dissolve the solid, and then cooled with approximately $20 \mathrm{~s}$ of sonication in an $\mathrm{H}_{2} \mathrm{O}$ bath (near ambient temperature). After standing undisturbed at $\mathrm{rt}$ for $\sim 10$ min, the vial was inverted to test for gelation for the first try. If no gel formed, the vial was heated again followed by sonication and resting undisturbed at rt before inverting to test for gelation for the second try.

Table S21. Sensing at $10,000 \mathrm{ppm}$.

\begin{tabular}{ccc}
\hline Trial & gel $1^{\text {st }}$ try? & $\begin{array}{c}\text { gel } 2^{\text {nd }} \\
\text { try? }\end{array}$ \\
\hline 1 & yes & -- \\
2 & yes & -- \\
3 & yes & - \\
4 & yes & - \\
5 & yes & - \\
\hline
\end{tabular}

Sensing at $\mathbf{4 0 0 0}$ ppm:

General Procedure - To an $8 \mathrm{~mL}$ vial, $0.66 \mathrm{~mL}$ of the $5000 \mathrm{ppm}$ lead paint solution in paint thinner was added along with $0.17 \mathrm{~mL} 0 \mathrm{ppm}$ paint solution for samples $1-4-$ or $-0.55 \mathrm{~mL} 6000$ ppm solution with $0.28 \mathrm{~mL} 0 \mathrm{ppm}$ paint solution for samples 5-6. Subsequently, $0.17 \mathrm{~mL}$ of a $19.1 \mathrm{mg}$ solution of 3 in $2.13 \mathrm{~mL} \mathrm{MeOH}$ was added. The vial was capped and sonicated for $20 \mathrm{~s}$ to ensure complete mixing. Subsequently, the vial was heated for $\sim 30-40 \mathrm{~s}$ to dissolve the solid, and then cooled with approximately $20 \mathrm{~s}$ of sonication in an $\mathrm{H}_{2} \mathrm{O}$ bath (near ambient temperature). After standing undisturbed at $\mathrm{rt}$ for $\sim 10 \mathrm{~min}$, the vial was inverted to test for gelation. If no gel formed, the vial was heated again followed by sonication and resting undisturbed at rt before inverting to test for gelation.

Table S22. Sensing at $4000 \mathrm{ppm}$.

\begin{tabular}{ccc}
\hline Trial & gel $\mathbf{1}^{\text {st }}$ try? & gel $\mathbf{2}^{\text {nd }}$ try? \\
\hline 1 & no & no \\
2 & no & yes \\
3 & no & no
\end{tabular}




\begin{tabular}{ccc}
4 & no & no \\
5 & no & yes \\
6 & no & $\begin{array}{c}\text { yes - very } \\
\text { weak gel }\end{array}$ \\
\hline
\end{tabular}

\section{Sensing at $\mathbf{3 0 0 0} \mathrm{ppm}$ :}

General Procedure - To an $8 \mathrm{~mL}$ vial, $0.50 \mathrm{~mL}$ of the $5000 \mathrm{ppm}$ lead paint solution in paint thinner was added along with $0.33 \mathrm{~mL} 0 \mathrm{ppm}$ paint solution for samples $1-3-$ or $-0.41 \mathrm{~mL} 6000$ ppm solution with $0.42 \mathrm{~mL} 0 \mathrm{ppm}$ paint solution for samples 4-5. Subsequently, $0.17 \mathrm{~mL}$ of a $19.1 \mathrm{mg}$ solution of 3 in $2.13 \mathrm{~mL} \mathrm{MeOH}$ was added. The vial was capped and sonicated for $20 \mathrm{~s}$ to ensure complete mixing. Subsequently, the vial was heated for $\sim 30-40$ s to dissolve the solid, and then cooled with approximately $20 \mathrm{~s}$ of sonication in an $\mathrm{H}_{2} \mathrm{O}$ bath (near ambient temperature). After standing undisturbed at $\mathrm{rt}$ for $\sim 10 \mathrm{~min}$, the vial was inverted to test for gelation. If no gel formed, the vial was heated again followed by sonication and resting undisturbed at rt before inverting to test for gelation.

Table S23. Sensing at $3000 \mathrm{ppm}$.

\begin{tabular}{ccc}
\hline Sample & gel $^{\mathbf{1}^{\text {st }}}$ try? & gel $\mathbf{2}^{\text {nd }}$ try? \\
\hline 1 & no & no \\
2 & no & no \\
3 & no & no \\
4 & no & no \\
5 & no & no \\
6 & no & no \\
\hline
\end{tabular}

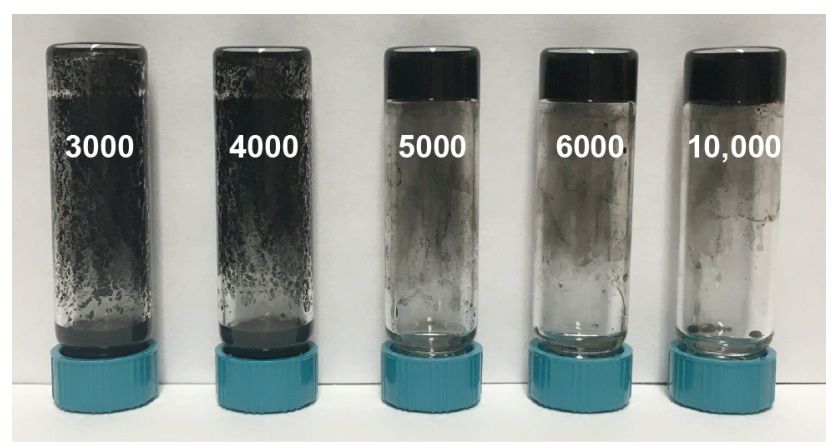

Figure S49. Gels form at concentrations of 5000 ppm lead in paint and above, while no gel forms at lower concentrations.

\section{B. Blind sensing with white oil-based paint}


Determining the cgc for in situ formation of 1d. A stock mixture of $5000 \mathrm{ppm}(5000 \mathrm{ppm}=$ $0.05 \%$ by weight $\mathrm{Pb}=6.6 \mathrm{mg} \mathrm{PbCO}_{3}$ in $995.1 \mathrm{mg}$ white oil-based paint) lead paint was prepared in $6 \mathrm{~mL}$ paint thinner. The lead-paint/paint thinner mixture was mixed well by sonicating for $\sim 60 \mathrm{~s}$ and vigorous shaking. Aliquots from this stock mixture were taken immediately after mixing. Since $\mathrm{PbCO}_{3}$ is insoluble, it is important to mix the stock mixture well prior to taking aliquots each time. An aliquot of $0.6 \mathrm{~mL}$ lead-paint mixture was taken and diluted with additional paint thinner $(0-0.4 \mathrm{~mL})$. Subsequently, $\sim 1.5 \mathrm{mg} 3$ was added as a solution in $\mathrm{MeOH}$ such that the total concentration of $\mathrm{MeOH}$ remains $\sim 17 \%$ by volume relative to the total volume of paint thinner.

General Procedure - To an $8 \mathrm{~mL}$ vial, $0.6 \mathrm{~mL}$ of the $5000 \mathrm{ppm}$ lead paint solution in paint thinner was added. If applicable, the solution was diluted with additional paint thinner. Subsequently, 3 was added as a solution in $\mathrm{MeOH}$. If applicable, additional $\mathrm{MeOH}$ was added prior to addition of the ligand solution. The vial was capped and sonicated for $20 \mathrm{~s}$ to ensure complete mixing. Once mixing appeared complete, the vial was heated to dissolve the solid, and subsequently cooled with approximately $20 \mathrm{~s}$ of sonication in an $\mathrm{H}_{2} \mathrm{O}$ bath (near ambient temperature). After standing undisturbed at $\mathrm{rt}$ for $\sim 10 \mathrm{~min}$, the vial was inverted to test for gelation.

Paint used:

white oil-based paint (soluble in paint thinner): Rust-Oleum, 7792 Gloss White, Protective Enamel

Table S24. Determining the cgc for in situ gel formation in the presence of paint. ${ }^{a, b}$

\begin{tabular}{ccccc}
\hline $\begin{array}{c}\text { total volume } \\
(\mathbf{m L})\end{array}$ & $\begin{array}{c}\text { additional paint } \\
\text { thinner }(\mathbf{m L})\end{array}$ & $\begin{array}{c}\text { volume } \\
\text { MeOH } \\
(\mathbf{m L})\end{array}$ & $\begin{array}{c}\text { gel } \mathbf{1}^{\text {st }} \\
\text { try? }\end{array}$ & $\begin{array}{c}\text { gel 2 } \\
\text { try? }\end{array}$ \\
\hline 0.72 & 0 & 0.12 & yes & -- \\
0.96 & 0.2 & 0.16 & yes & -- \\
$1.08^{\mathrm{c}}$ & 0.3 & $\mathbf{0 . 1 8}$ & yes & - \\
1.20 & 0.4 & 0.20 & no & no \\
\hline
\end{tabular}

a) For each sample, used $0.6 \mathrm{~mL}$ of a $6.6 \mathrm{mg} \mathrm{PbCO}_{3}$ in $995.1 \mathrm{mg}$ paint solution in $6 \mathrm{~mL}$ paint thinner. b) $0.16 \mathrm{~mL}$ of a solution containing $7.5 \mathrm{mg} 3$ in $0.6 \mathrm{~mL} \mathrm{MeOH}$ was used. Additional $\mathrm{MeOH}$ was added to sample vials to get to $17 \%$ by volume $\mathrm{MeOH}$. c) This experiment was repeated two times. A gel formed each time.

\section{Sensing at $\mathbf{4 0 0 0}$ and $\mathbf{3 0 0 0}$ ppm:}

General Procedure - To an $8 \mathrm{~mL}$ vial, an appropriate amount of the $5000 \mathrm{ppm}$ lead paint solution in paint thinner was added along with additional $0 \mathrm{ppm}$ paint solution $(998.2 \mathrm{mg}$ in $6 \mathrm{~mL}$ paint thinner) and $0.3 \mathrm{~mL}$ paint thinner. Subsequently, $0.06 \mathrm{~mL} \mathrm{MeOH}$ was added, followed by $0.12 \mathrm{~mL}$ of a $7.7 \mathrm{mg}$ solution of $3 \mathrm{in} 0.16 \mathrm{~mL} \mathrm{MeOH}$. The vial was capped and sonicated for $20 \mathrm{~s}$ to ensure complete mixing. Subsequently, the vial was heated for $\sim 30-40 \mathrm{~s}$ to dissolve the solid, and then cooled with approximately $20 \mathrm{~s}$ of sonication in an $\mathrm{H}_{2} \mathrm{O}$ bath (near ambient temperature). After standing undisturbed at $\mathrm{rt}$ for $\sim 10 \mathrm{~min}$, the vial was inverted to test for gelation. If no gel formed, the vial was heated again followed by sonication and resting undisturbed at rt before inverting to test for gelation. 
Table S25. Sensing at 4000 and 3000 ppm.

\begin{tabular}{ccccc} 
ppm & $\begin{array}{c}\text { amount 5000 } \\
\text { ppm solution }\end{array}$ & $\begin{array}{c}\text { amount 0 ppm } \\
\text { paint solution }\end{array}$ & $\begin{array}{c}\text { Additional paint } \\
\text { thinner }\end{array}$ & gel? \\
\hline 4000 & 0.36 & 0.24 & 0.3 & $\mathrm{no}^{\mathrm{c}}$ \\
3000 & 0.48 & 0.12 & 0.3 & $\mathrm{no}^{\mathrm{c}}$ \\
$3000^{\mathrm{a}}$ & 0.48 & 0.12 & 0.3 & $\mathrm{no}^{\mathrm{c}}$ \\
$3000^{\mathrm{b}}$ & 0.48 & 0.12 & 0.3 & $\mathrm{no}^{\mathrm{c}}$ \\
\hline
\end{tabular}

a) The sample was tested after $1 \mathrm{~min}$, instead of $10 \mathrm{~min}$. b) The sample was tested after $1 \mathrm{~h}$, instead of 10 min. c) No gel formed after two gel-formation attempts at the indicated wait time.

\section{Blind Sensing:}

To remove user bias, a blind sensing experiment was performed. The operator added the ligand and performed the sensing procedure, but was unaware of the concentration of each sample until after the experiment was complete.

Stock solutions: $5000 \mathrm{ppm}\left(6.6 \mathrm{mg} \mathrm{PbCO}_{3}, 995.1 \mathrm{mg}\right.$ paint, $6 \mathrm{~mL}$ paint thinner); $10,000 \mathrm{ppm}$ (12.8 $\mathrm{mg} \mathrm{PbCO}_{3}, 999.7 \mathrm{mg}$ paint, $6 \mathrm{~mL}$ paint thinner); $0 \mathrm{ppm}$ (998.2 $\mathrm{mg}$ paint, $6 \mathrm{~mL}$ paint thinner); 3 (24 $\mathrm{mg} 3$ in $2.88 \mathrm{~mL} \mathrm{MeOH)}$

Sample preparation:

10,000 ppm: $0.6 \mathrm{~mL} 10000 \mathrm{ppm}$ stock solution, $0.3 \mathrm{~mL}$ paint thinner

6000 ppm: $0.36 \mathrm{~mL} 10000 \mathrm{ppm}$ stock solution, $0.24 \mathrm{~mL} 0 \mathrm{ppm}$ paint solution, $0.3 \mathrm{~mL}$ paint thinner

5000 ppm: $0.6 \mathrm{~mL} 5000 \mathrm{ppm}$ stock solution, $0.3 \mathrm{~mL}$ paint thinner

4000 ppm: $0.48 \mathrm{~mL} 5000 \mathrm{ppm}$ stock solution, $0.12 \mathrm{~mL} 0 \mathrm{ppm}$ paint solution, $0.3 \mathrm{~mL}$ paint thinner 3000 ppm: $0.36 \mathrm{~mL} 5000 \mathrm{ppm}$ stock solution, $0.24 \mathrm{~mL} 0 \mathrm{ppm}$ paint solution, $0.3 \mathrm{~mL}$ paint thinner General Procedure - To an $8 \mathrm{~mL}$ vial with an unknown (to the operator) concentration of lead paint solution, $0.18 \mathrm{~mL} 3$ stock solution was added. The vial was capped and sonicated for $20 \mathrm{~s}$ to ensure complete mixing. Subsequently, the vial was heated for $\sim 30-40 \mathrm{~s}$ to dissolve the solid, and then cooled with approximately $20 \mathrm{~s}$ of sonication in an $\mathrm{H}_{2} \mathrm{O}$ bath (near ambient temperature). After standing undisturbed at $\mathrm{rt}$ for $\sim 10 \mathrm{~min}$, the vial was inverted to test for gelation. If no gel formed, the vial was heated again followed by sonication and resting undisturbed at $\mathrm{rt}$ before inverting to test for gelation.

Table S26. Blind sensing at varying $\mathrm{Pb}$ concentrations (3000-10,000 ppm)

\begin{tabular}{cccc}
\hline Vial \# & gel $\mathbf{1}^{\text {st }}$ try & gel $\mathbf{2}^{\text {nd }}$ try $?^{\text {concentration }}$ & $\begin{array}{c}\text { con }) \\
(\mathbf{p p m})\end{array}$ \\
\hline 1 & yes & -- & 5000 \\
2 & no & no & 3000 \\
3 & no & no & 4000 \\
4 & yes & -- & 10,000 \\
5 & no & no & 4000 \\
6 & yes & -- & 6000 \\
7 & yes & -- & 5000 \\
8 & no & no & 3000 \\
9 & yes & -- & 5000 \\
10 & no & no & 4000 \\
11 & yes & -- & 5000 \\
12 & yes & -- & 10,000 \\
\hline
\end{tabular}




\section{$\mathrm{XX}$. Developing a sensor for $\mathrm{Pb}$ at $\mathbf{5 0 0 0} \mathrm{ppm}$ in dry paint}

Dried pink latex based paint was used for these experiments.

\section{A. Solvent screen for gel formation}

Several solvents that had been shown to solubilize the pink paint were tested for the viability of gel formation.

General Procedure - To an $8 \mathrm{~mL}$ vial containing $\sim 95 \mathrm{mg}$ dry paint and $\sim 6 \mathrm{mg} \mathrm{PbCO}_{3}, 0.4 \mathrm{~mL}$ solvent was added, and the vial was capped and sonicated to dissolve the paint. Subsequently, $\sim 1.5 \mathrm{mg} 3$ was added. The vial was capped and sonicated for $20 \mathrm{~s}$ to ensure complete mixing. Subsequently, the vial was heated for $\sim 30-40 \mathrm{~s}$ to dissolve the solid, and then cooled with approximately $20 \mathrm{~s}$ of sonication in an $\mathrm{H}_{2} \mathrm{O}$ bath (near ambient temperature). After standing undisturbed at rt for $\sim 10 \mathrm{~min}$, the vial was inverted to test for gelation.

Table S27. Solvent screening for dry paint sensing.

\begin{tabular}{cc}
\hline solvent & gel? \\
\hline $\mathrm{MEK}$ & no \\
$\mathrm{CH}_{2} \mathrm{Cl}_{2}$ & no \\
$\mathrm{MeOH}$ & yes \\
EtOAc & no \\
paint thinner & no \\
acetone & no \\
\hline
\end{tabular}

\section{B. Determining the in situ cgc for sensing dry paint in $\mathrm{MeOH}$ at $5000 \mathrm{ppm}$, and testing the sensor at $\mathbf{3 0 0 0} \mathrm{ppm}$}

A stock solution of $\mathrm{PbCO}_{3}$ in $\mathrm{MeOH}$ was prepared $\left(7.4 \mathrm{mg} \mathrm{PbCO}_{3}\right.$ in $6 \mathrm{~mL} \mathrm{MeOH}$ ). The lead$\mathrm{MeOH}$ mixture was mixed well by sonicating for $\sim 60 \mathrm{~s}$ and vigorous shaking. Aliquots from this stock mixture were taken immediately after mixing. Since $\mathrm{PbCO}_{3}$ is insoluble, it is important to mix the stock mixture well prior to taking aliquots each time. An aliquot of $0.27 \mathrm{~mL}$ lead-MeOH mixture was taken and diluted with additional $\mathrm{MeOH}(0.9-1.1 \mathrm{~mL})$. Subsequently, $1.5 \mathrm{mg} 3$ was added as a solution in $0.09 \mathrm{~mL} \mathrm{MeOH}$.

General Procedure - To an $8 \mathrm{~mL}$ vial containing $\sim 47 \mathrm{mg}$ dry pink paint, $0.27 \mathrm{~mL}$ of the $\mathrm{PbCO}_{3}$ ppm lead solution in $\mathrm{MeOH}$ was added for $5000 \mathrm{ppm}$ samples, $0.16 \mathrm{~mL}$ for the $3000 \mathrm{ppm}$ samples. The solution was diluted with additional $\mathrm{MeOH}$. At this point the vial was sonicated for $\sim 30 \mathrm{~s}$ to dissolve the paint as much as possible. Subsequently, 3 was added as a solution in $\mathrm{MeOH}$. The vial was capped and sonicated for $20 \mathrm{~s}$ to ensure complete mixing. Once mixing appeared complete, the vial was heated to dissolve the solid, and subsequently cooled with approximately $20 \mathrm{~s}$ of sonication in an $\mathrm{H}_{2} \mathrm{O}$ bath (near ambient temperature). After standing undisturbed at rt for $\sim 10 \mathrm{~min}$, the vial was inverted to test for gelation. If no gel formed, the vial was heated again followed by sonication and resting undisturbed at $r$ before inverting to test for gelation. 
Table S28. Determining the cgc for in situ gel formation at $5000 \mathrm{ppm}$ from dry paint.

\begin{tabular}{cccc}
\hline $\begin{array}{c}\text { Pb concentration } \\
(\mathbf{p p m})\end{array}$ & $\begin{array}{c}\text { total volume } \\
\text { MeOH }(\mathbf{m L})\end{array}$ & $\begin{array}{c}\text { gel } \mathbf{1}^{\text {st }} \\
\text { try? }\end{array}$ & $\begin{array}{c}\text { gel 2 }^{\text {nd }} \\
\text { try? }\end{array}$ \\
\hline 5000 & 1.26 & yes & - \\
$5000^{\text {a }}$ & 1.36 & yes & -- \\
5000 & 1.46 & no & no \\
$3000^{b}$ & 1.36 & no & no
\end{tabular}

a) This experiment was repeated two times. A gel formed each time. b) This experiment was repeated two times. No gel formed either time. 
$X X I$. Effect of excess ligand and $\mathrm{Cu}(\mathrm{OAc})_{2}$ on gel formation

\section{A. Effect of excess ligand on gel formation}

Using the same approach as described above, the cgc for in situ gel formation in $\mathrm{PT} / \mathrm{MeOH}$ in the absence of paint was determined to be $1.1 \mathrm{mg} \mathrm{PbCO} / \mathrm{mL}$ paint thinner:MeOH $(17 \% \mathrm{MeOH}$ by volume) when using a 1:2 $\mathrm{Pb}: 3$ ratio.

At this concentration of $\mathrm{Pb}$ in the paint thinner/MeOH, the effect of additional ligand on gel formation was examined.

General Procedure - To an $8 \mathrm{~mL}$ vial containing the appropriate amount of a $\mathrm{PbCO}_{3}$ solution (8.6 $\mathrm{mg} \mathrm{PbCO}_{3}$ in $6.5 \mathrm{~mL}$ paint thinner) was added and diluted to a total volume of $0.5 \mathrm{~mL}$ paint thinner. Subsequently, $0.1 \mathrm{~mL}$ of a solution of $3 \mathrm{in} \mathrm{MeOH}$ was added $(1.5 \mathrm{mg} / 0.1 \mathrm{~mL}$ for 2 equiv, $3 \mathrm{mg} / 0.1 \mathrm{~mL}$ for 3 equiv, $6 \mathrm{mg} / 0.1 \mathrm{~mL}$ for 6 equiv). The vial was capped and sonicated for $20 \mathrm{~s}$ to ensure complete mixing. Once mixing appeared complete, the vial was heated to dissolve the solid, and subsequently cooled with approximately $20 \mathrm{~s}$ of sonication in an $\mathrm{H}_{2} \mathrm{O}$ bath (near ambient temperature). After standing undisturbed at $\mathrm{rt}$ for $\sim 10 \mathrm{~min}$, the vial was inverted to test for gelation. If no gel formed, the vial was heated again followed by sonication and resting undisturbed at rt before inverting to test for gelation.

Table S29. Effect of additional ligand on gelation at the cgc.

\begin{tabular}{cccc}
\hline Equiv 3 (ligand) & $\begin{array}{c}\text { Amount } \\
\text { of Pb } \\
\text { present } \\
(\mathbf{m g})\end{array}$ & $\begin{array}{c}\text { gel 1 } \\
\text { try? }\end{array}$ & $\begin{array}{c}\text { gel 2 } \\
\text { try? }\end{array}$ \\
\hline $\mathbf{2}$ & $\mathbf{0 . 6 6}$ & yes & -- \\
2 & 0.39 & no & no \\
\hline 4 & $\mathbf{0 . 6 6}$ & no & yes \\
4 & 0.39 & no & no \\
6 & 0.66 & no & no \\
\hline
\end{tabular}

\section{B. Effect of $\mathrm{Cu}(\mathrm{OAc})_{2}$ on gel formation}

A stock mixture of $\mathrm{PbCO}_{3}$ in paint thinner was prepared (8.6 $\mathrm{mg} \mathrm{PbCO}_{3}$ in $6.5 \mathrm{~mL}$ paint thinner). The lead-paint thinner mixture was mixed well by sonicating for $\sim 60 \mathrm{~s}$ and vigorous shaking. Aliquots from this stock mixture are taken immediately after mixing. Since $\mathrm{PbCO}_{3}$ is insoluble, it is important to mix the stock mixture well prior to taking aliquots each time. An aliquot of $0.5 \mathrm{~mL}$ lead-paint thinner mixture was used for each sample.

General Procedure - To an $8 \mathrm{~mL}$ vial, $0.5 \mathrm{~mL}$ of the $\mathrm{PbCO}_{3} \mathrm{ppm}$ lead solution in paint thinner was added, followed by $\mathrm{Cu}(\mathrm{OAc})_{2}(1.4 \mathrm{mg}$ for a $1: 1 \mathrm{Cu}: \mathrm{Pb}$ ratio, and $0.3 \mathrm{mg}$ for $1: 5 \mathrm{Cu}: \mathrm{Pb}$ ratio). Finally, 3 was added as a solution in $\mathrm{MeOH}(1.5 \mathrm{mg}$ for 2 equiv $3: \mathrm{Pb}$ and $3.0 \mathrm{mg} 4$ equiv $3: \mathrm{Pb})$. The vial was capped and sonicated for $20 \mathrm{~s}$ to ensure complete mixing. Once mixing appeared complete, the vial was heated to dissolve the solid, and subsequently cooled with approximately $20 \mathrm{~s}$ of sonication in an $\mathrm{H}_{2} \mathrm{O}$ bath (near ambient temperature). After standing undisturbed at $\mathrm{rt}$ for $\sim 10 \mathrm{~min}$, the vial was inverted to test for gelation. If no gel formed, the vial was heated again followed by sonication and resting undisturbed at rt before inverting to test for gelation. 
Table S30. Sensing at varying ratios of $\mathrm{Cu}: \mathrm{Pb}$ with different amounts of ligand added.

\begin{tabular}{|c|c|c|c|}
\hline Cu:Pb ratio & $\begin{array}{c}\text { Equiv } 3 \\
\text { (relative } \\
\text { to } \mathrm{Pb} \text { ) }\end{array}$ & $\begin{array}{c}\text { gel } 1^{\text {st }} \\
\text { try? }\end{array}$ & $\begin{array}{c}\text { gel } 2^{\text {nd }} \\
\text { try? }\end{array}$ \\
\hline $1: 1$ & 2 & no & no \\
\hline $1: 1$ & 4 & no & no \\
\hline $1: 5$ & 2 & no & no \\
\hline $1: 5$ & 4 & no & yes \\
\hline
\end{tabular}




\section{Comparing the gel-based sensor to LeadCheck ${ }^{\text {TM }}$}

To show that the detection range of our gelator is much narrower than that of LeadCheck ${ }^{\mathrm{T}}$ by $3 \mathrm{M}^{\mathrm{Tm}}$, $3.9 \mathrm{mg} \mathrm{PbCO}{ }_{3}$ was added to $996 \mathrm{mg}$ dried pink paint. The paint was dissolved in acetone, and the sample sonicated to evenly distribute the lead in solution. The solution was then transferred to a small petri dish and the solvent evaporated at rt. Subsequently, the paint was scratched with a spatula prior to testing the paint with a swab of $3 \mathrm{M}^{\mathrm{TM}}$ LeadCheck ${ }^{\text {TM }}$ following the instructions provided with the swab. The swab immediately turned orange/red, indicating the presence of lead. As a control, a separate swab was used to test a Pb-free sample of pink paint. As expected, no red color formed.

Additionally, $48.6 \mathrm{mg}$ of the paint was transferred to an $8 \mathrm{~mL}$ vial. $\mathrm{MeOH}(1.27 \mathrm{~mL})$ was added, and the vial sonicated for $\sim 60 \mathrm{~s}$ to dissolve the paint. Subsequently, $0.09 \mathrm{~mL}$ of a solution of 3 in $\mathrm{MeOH}$ (12.1 mg 3 in $1.44 \mathrm{~mL} \mathrm{MeOH}$ ) was added. The vial was capped and sonicated for $20 \mathrm{~s}$ to ensure complete mixing. Once mixing appeared complete, the vial was heated to dissolve the solid, and subsequently cooled with approximately $20 \mathrm{~s}$ of sonication in an $\mathrm{H}_{2} \mathrm{O}$ bath (near ambient temperature). After standing undisturbed at it for $\sim 10 \mathrm{~min}$, the vial was inverted to test for gelation. Gel formation was not observed.

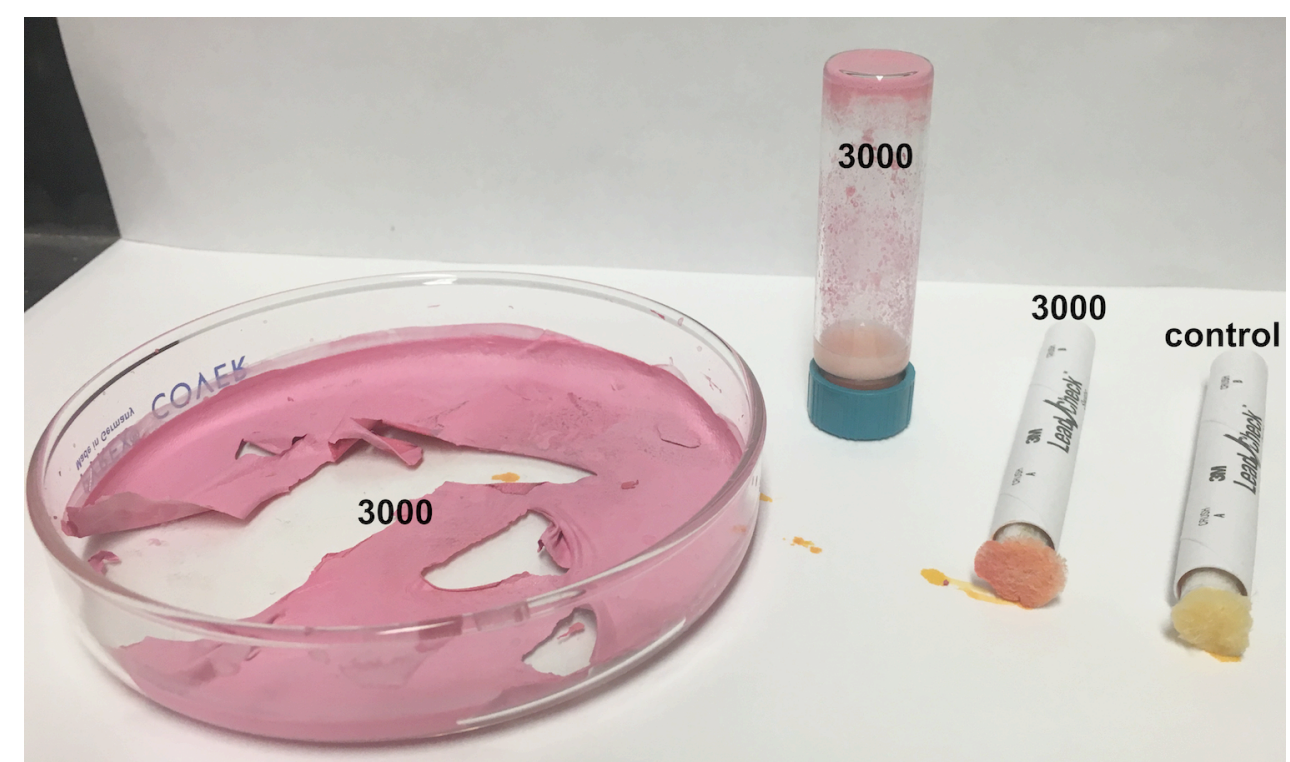

Figure S50. Comparison of our gel-based sensor with $3000 \mathrm{ppm}$ lead paint sample gives a negative result for lead, while $3 \mathrm{M}^{\mathrm{TM}}$ LeadCheck ${ }^{\mathrm{TM}}$ provides a positive red signal in the presence of 3000 ppm lead. Pictured L to R: petri dish with 3000 ppm lead paint; negative result for lead from a gel experiment; red coloration of the LeadCheck ${ }^{\mathrm{TM}}$ swab indicating presence of $\mathrm{Pb}$; control swab tested on lead-free paint. 


\section{References}

[1] Ren, H.; Wu, C.; Ding, X.; Chen, X.; Shi, F. Org. Biomol. Chem. 2012, 10, 8975.

[2] Trindade, T.; O'Brien, P.; Zhang, X.-M.; Motevalli, M. J. Mater. Chem. 1997, 7, 1011.

[3] Ng, S. W. Acta Cryst. 1999, C55, IUC9900091.

[4] Mohammadnezhad, G.; Ghanbarpour, A. R.; Amini, M. M.; Ng, S. W. Acta Cryst. 2010, E66, m529.

[5] Mishra, A. K.; Kaushik, N. K. Spectrochim. Acta Part A 2008, 69, 842.

[6] Materials Studio, version 6.0. http://accelrys.com/products/materials-studio/index.html.

[7] Mohammadnezhad, G.; Amini, M. M.; Langer, V. Acta Cryst. 2010, C66, m44.

[8] Chen, M.; Fulton, J. R.; Hitchcock, P. B.; Johnstone, N. C.; Lappert, M. F.; Protchenko, A. V. Dalton Trans. 2007, 2770.

[9] Zheng, K.-K.; Fang, X.; Yu, H.-Y.; Ke, H.; Wang, J.-D. Acta Cryst. 2010, E66, m1700.

[10] Klevtsova, R. F.; Sankova, E. A.; Kokina, T. E.; Glinskaya, L. A.; Larionov, S. V. J. Struct. Chem. 2008, 49, 117.

[11] Marandi, F.; Safarifard, V.; Morsali, A.; Fun, H.-K. J. Coord. Chem. 2011, 64, 3781.

[12] Yang, J.; Ma, J.-F.; Liu, Y.-Y.; Ma, J.-C.; Batten, S. R. Cryst. Growth Des. 2009, 9, 1894.

[13] Feng, T.-J. Acta Cryst. 2011, E67, m935. 